\title{
ARTERIOGENIC REVASCULARIZATION DOES NOT INDUCE VASCULAR FUNCTION IMPAIRMENT
}

\author{
A Thesis \\ presented to \\ the Faculty of California Polytechnic State University \\ San Luis Obispo
}

\author{
In Partial Fulfillment \\ of the Requirements for the Degree \\ Master of Science in Biomedical Engineering
}

by

Matthew David Yocum

March 2009 
(C) 2009

Matthew David Yocum

ALL RIGHTS RESERVED 


\section{COMMITTEE MEMBERSHIP}

TITLE:

Arteriogenic Revascularization Does Not Induce Vascular

Function Impairment

AUTHOR: $\quad$ Matthew David Yocum

DATE SUBMITTED: $\quad$ March 2009

COMMITTEE CHAIR: $\quad$ Dr. Trevor Cardinal

COMMITTEE MEMBER: Dr. Kristen O’Halloran Cardinal

COMMITTEE MEMBER: Dr. Lily Laiho 


\begin{abstract}
Arteriogenic Revascularization Does Not Induce Vascular Function Impairment Matthew Yocum
\end{abstract}

Functional hyperemia and arteriolar vasodilation are impaired with chronic ischemia. We sought to examine the impact of chronic ischemia on collateral artery function. For this we used two hindlimb ischemia models to dissect the impact of different repair processes on collateral function. Ligation of the femoral artery increases shear stress in the muscular branch and results in outward remodeling and arteriogenesis. In contrast, resection of the femoral artery proximal to the muscular branch induces blood flow divergence and neutral remodeling along with expectedly greater hypoxia and inflammation. On day 14 after each surgery the diameter of the muscular branch was measured using sidestream dark field (SDF) imaging before and after gracilis muscle stimulation. A slight, but not statistically significant, impairment in functional vasodilation was observed in ligated mice $(69 \pm 10 \%$ average diameter increase compared to $74 \pm 7 \%$ average diameter increase). Resected mice exhibited slightly (not statistically significant) enhanced collateral artery functional vasodilation $(104 \pm 16 \%$ average diameter increase) but were also refractory to the restoration of resting vascular tone following the cessation of stimulation. Outward remodeling did not significantly impair vascular function, whereas neutral remodeling and tissue hypoxia induced impaired vascular tone.

Keywords: Vascular Function, Ischemia, Arteriogenesis, Mouse, SDF Imaging, Gracilis, Peripheral Artery Disease. 


\section{ACKNOWLEDGEMENTS}

I would like to acknowledge Trevor Cardinal for his help and guidance, Mollie McDonnell for her patience, my parents (David Yocum and Alicia Eyherabide) for their life-long support, Stephanie Wood for giving me hope, Allyse Alex for guiding me through my first surgery and Cal Poly for providing me the opportunity to contribute to exciting research.

The following work is dedicated to all of the mice that helped progress our understanding of ischemia.

"Every experiment proves something. If it doesn't prove what you wanted it to prove, it proves something else."

-Anonymous 


\section{TABLE OF CONTENTS}

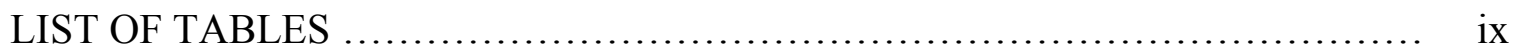

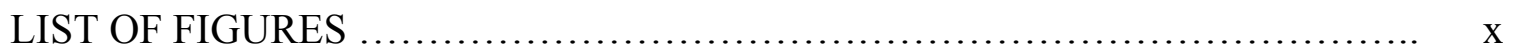

\section{CHAPTER}

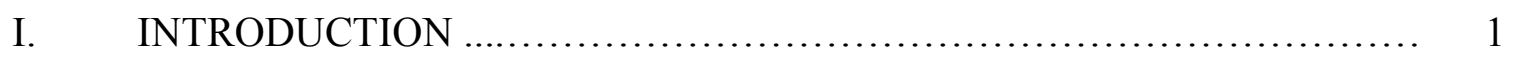

1.1. Circulatory Function and Dysfunction ...................... 1

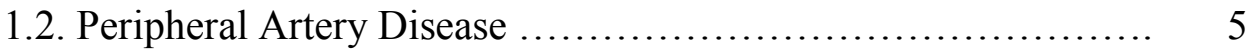

1.3. Mechanisms of Vascular Growth and Function ................. 8

1.3.1. Arteriogenesis ................................ 8

1.3.2. Angiogenesis .................................. 11

1.3.3. Blood Flow Control Pathways .................... 13

1.3.4. Interrelation Between Vascular Growth and Function

During Ischemic Repair ......................... 14

1.4. Mouse Models of Hindlimb Ischemia ....................... 15

1.4.1. Distinctions Between Animal Ischemia Models and

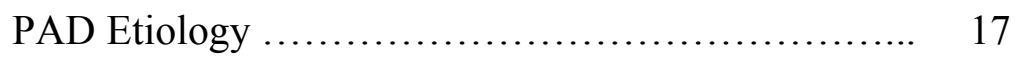

1.4.2. Femoral Artery Ligation Model ................... 20

1.4.3. Femoral Artery Resection Model ..................... 24

1.5. Sidestream Dark Field Imaging $\ldots \ldots \ldots \ldots \ldots \ldots \ldots \ldots \ldots \ldots \ldots . \ldots . \ldots . \ldots . \ldots$

1.6. Objectives and Hypotheses ............................... 29

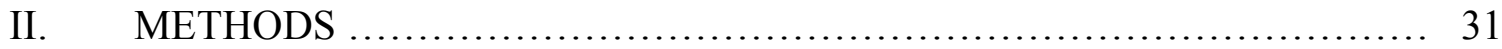


2.1. Housing for Mice

2.2. Vascular Function Experiments on Mice Not Surgically Altered .. 31

2.3. Femoral Artery Ligation Surgical Protocol .................... 35

2.4. Femoral Artery Resection Surgical Protocol ................... 36

2.5. Vascular Function Experiments on Ischemic Mice .............. 37

2.6. SDF Imaging Analysis Protocol Using AVA

(Automated Vascular Analysis) ......................... 38

2.7. Statistical Analysis ....................................... 41

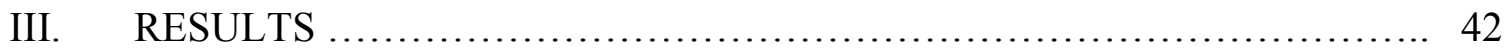

3.1. Functional Hyperemia in Healthy Animals ................... 42

3.2. Outward Remodeling and Vasodilation Following

Arteriogenic Ischemia ................................ 44

3.3. Arterial Resting Tone and Vasodilation Following

Non-Arteriogenic Ischemia $\ldots \ldots \ldots \ldots \ldots \ldots \ldots \ldots \ldots \ldots \ldots . \ldots 4$

IV. DISCUSSION .................................................... 52

4.1. Restatement of Experimental Objectives .................... 52

4.2. Vasodilatory Response in Healthy Mice .................... 52

4.3. SDF Imaging Protocol Modifications .......................... 53

4.3.1. Potential Protocol Adaptations yet to be Addressed ...... 56

4.3.2. Future Protocol Improving Modifications .............. 59

4.3.3. The Importance of Limb Order and

Potential Considerations .......................... 60

4.4. Flow Control/Arterial Vasodilation During 
Remodeling of the Muscular Branch Artery

4.4.1. Arteriogenesis 61

4.4.2. Vasodilation in Arteriogenic Conditions

(Femoral Artery Ligation) 62

4.4.3. A Comparison of Hindlimb Ischemia Models to

Clarify Vasodilation Results 63

4.4.4. Remodeling Following Non-Arteriogenic Ischemia 66

4.4.5. Vasodilation Following Non-Arteriogenic Ischemia ..... 69

4.5. Modifications for Subsequent Non-Arteriogenic

Ischemia Models 71

4.6. Future Investigation 73

REFERENCES 76

\section{APPENDICES}

A. Vascular Function and Blood Flow Equations ................... 84

B. Husbandry Practices ................................... 85

C. SDF Imaging Experimental Checklist $\ldots \ldots \ldots \ldots \ldots \ldots \ldots \ldots \ldots \ldots . . \ldots 6$

D. Femoral Artery Ligation Checklist .......................... 87

E. Femoral Artery Resection Checklist ........................... 88

F. Muscular branch functional hyperemia data for healthy mice ........ 89

G. Muscular branch functional hyperemia data for ligated mice ......... 90

H. Muscular branch functional hyperemia data for resected mice ........ 91 


\section{LIST OF TABLES}

Table $\quad$ Page

1. Muscular branch functional hyperemia data for healthy mice ............. 89

2. Muscular branch functional hyperemia data for ligated mice .............. 90

3. Muscular branch functional hyperemia data for resected mice ............. 91 


\section{LIST OF FIGURES}

Figure

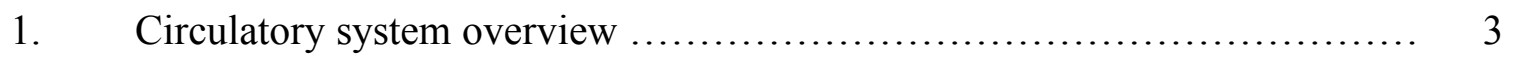

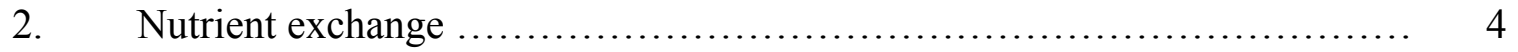

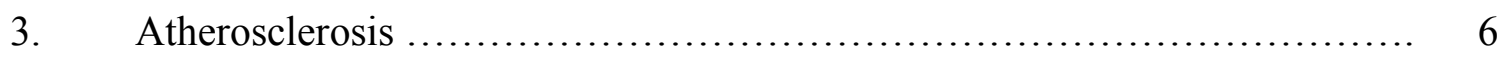

4. Arterial layers and outward remodeling mechanisms $\ldots \ldots \ldots \ldots \ldots \ldots \ldots \ldots . . . \ldots$

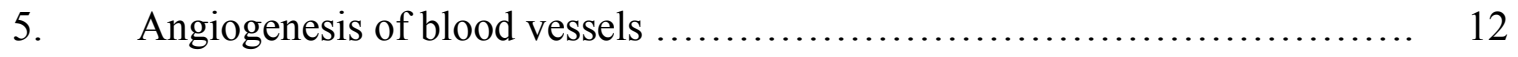

6. Arteriole blood flow control through vasodilation and vasoconstriction ....... 13

7. Generalized juxtaposition of surgical models .......................... 17

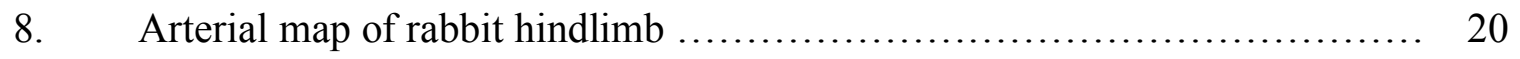

9. Diagram of the femoral artery ligation model ......................... 21

10. Hindlimb perfusion changes following femoral artery ligation $\ldots \ldots \ldots \ldots \ldots .23$

11. Arteriogenesis following femoral artery ligation $\ldots \ldots \ldots \ldots \ldots \ldots \ldots \ldots \ldots .24$

12. A diagram of the femoral artery resection model for hindlimb ischemia ....... 25

13. Diagram of SDF imaging mechanism .............................. 28

14. Digital video data examples from OPS and SDF imaging ................. 29

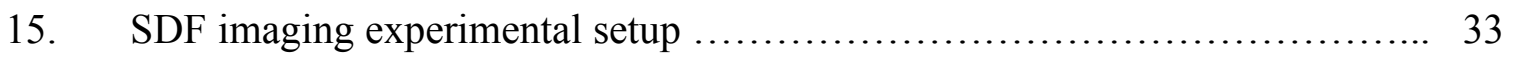

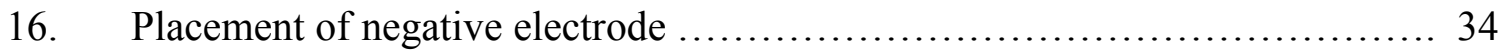

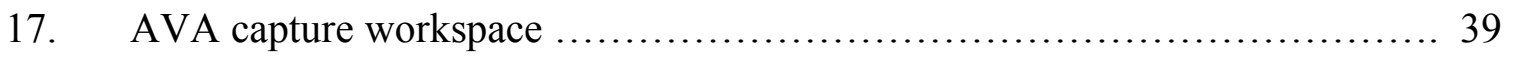

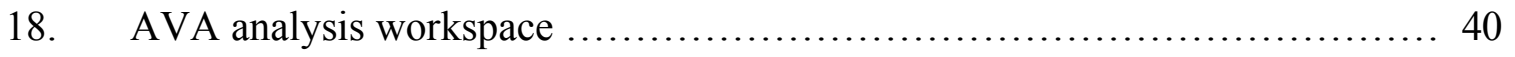

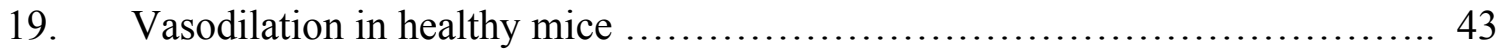

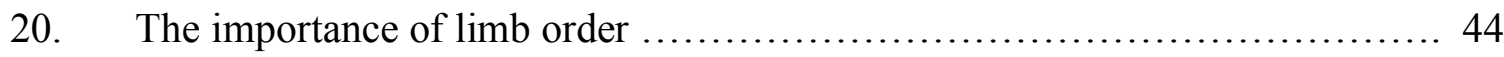

21. Arteriogenesis of the muscular branch artery in ligated mice $\ldots \ldots \ldots \ldots \ldots \ldots . . \ldots 5$ 
22. Vasodilation of the muscular branch artery in ligated mice .................. 46

23. Images of muscular branch artery changes in ligated mice $\ldots \ldots \ldots \ldots \ldots \ldots \ldots .47$

24. Neutral remodeling of the muscular branch artery in resected mice ........... 48

25. Vasodilation of the muscular branch artery in resected mice $\ldots \ldots \ldots \ldots \ldots \ldots . .50$

26. Images of muscular branch artery changes in resected mice ................ 51

27. Inconsistent data from original imaging protocol ......................... 55

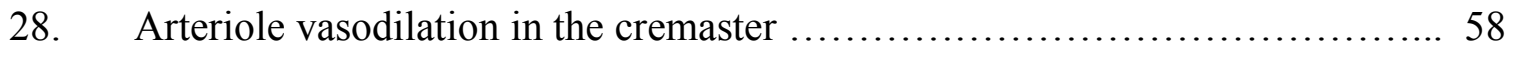

29. Image of turbulent flow in the muscular branch artery of resected mice ....... 67

30. Blood flow changes following femoral artery resection $\ldots \ldots \ldots \ldots \ldots \ldots \ldots \ldots . \ldots 6$

31. Remodeling of gracilis-perfusing vessels following femoral artery resection ... 69

32. A modified femoral artery resection model $\ldots \ldots \ldots \ldots \ldots \ldots \ldots \ldots \ldots \ldots \ldots \ldots$

33. SDF imaging experimental checklist ............................... 86

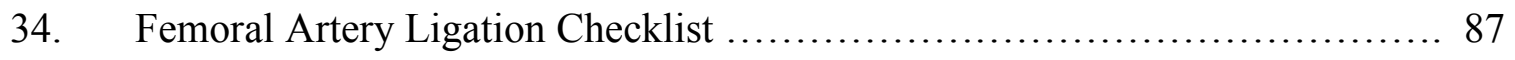

35. Femoral Artery Resection Checklist ................................ 88 


\section{INTRODUCTION}

\subsection{Circulatory FunCTION AND DYSFUNCTION}

The circulatory system consists of the heart as well as the arteries, veins and capillaries that perfuse all bodily tissues to deliver key metabolic nutrients like oxygen and glucose (Figure 1A) [1]. The most important function of the circulatory system is to transport these nutrients to functioning tissues and deliver metabolic waste to the liver, lungs or kidneys for excretion or degradation (the circulatory system thus plays an important role in homeostasis including temperature and $\mathrm{pH}$ control) [1]. This nutrient/waste exchange is mediated by the heart, which pumps deoxygenated blood to the lungs, where carbon dioxide is released and oxygen binds to hemoglobin-containing red blood cells (Figure 2A) [1]. After leaving the lungs, oxygenated blood is then transported to the heart and pumped through the arteries to the systemic tissues (Figure 1B) [1]. The exchange of metabolites such as oxygen, carbon dioxide and glucose occurs via convection and diffusion (promoted by hydrodynamic and osmotic pressure gradients) and is facilitated by the thin, single cell thick, capillary walls where exchange occurs (Figure 1C) [2]. This thin wall between the tissues or alveoli in the lungs and the circulating blood assists metabolite diffusion, because the flux of migrating gaseous or aqueous molecules is inversely related to the distance of the crossed barrier [2]. Further, diffusion and convection, the mechanisms of nutrient exchange in the tissues, are mediated by a balance between blood pressure (which also promotes blood filtration in coordination with the lymphatic system) and osmotic pressure (Figure 2B) $[1,2]$. Coordinating these 
processes to maintain proper nutrient trafficking and vascular function is vital for the health of all tissues in order to sustain sufficient ATP production required for cellular activity and viability $[1,2]$. It is also imperative that the circulatory system not only supply sufficient blood flow to tissues during rest, but that it is also amenable to adjusting blood flow to specific tissues as metabolic demands shift [3-5]. For example, after a meal the digestive system, particularly the intestine, requires an increase in blood flow to facilitate digestion and absorption. Normally the intestine receives $20 \%$ of the cardiac output, however, after a meal this amount increases 2-fold [6].

Mechanistically, localized blood flow control is mediated primarily through arterial lumen expansion and contraction (vasodilation and vasoconstriction, respectively) [3, 4]. These adjustments in arterial diameter result in large changes in blood flow, because blood flow is directly related to the lumen radius to the power of 4 (Poisseuille's Law, Appendix A) [7]. Thus, vasodilation and vasoconstriction are effective methods for specific tissue blood flow control. Overall, blood and nutrient delivery are affected locally by arterial expansion/contraction and diffusion distance between the tissues and blood, as well as generally by cardiac output. Given the important functions of the circulatory system, local vascular complications that affect arterial relaxation/constriction or that increase tissue capillary distance can be especially detrimental to systemic health, because they inhibit proper nutrient delivery [1-5]. 

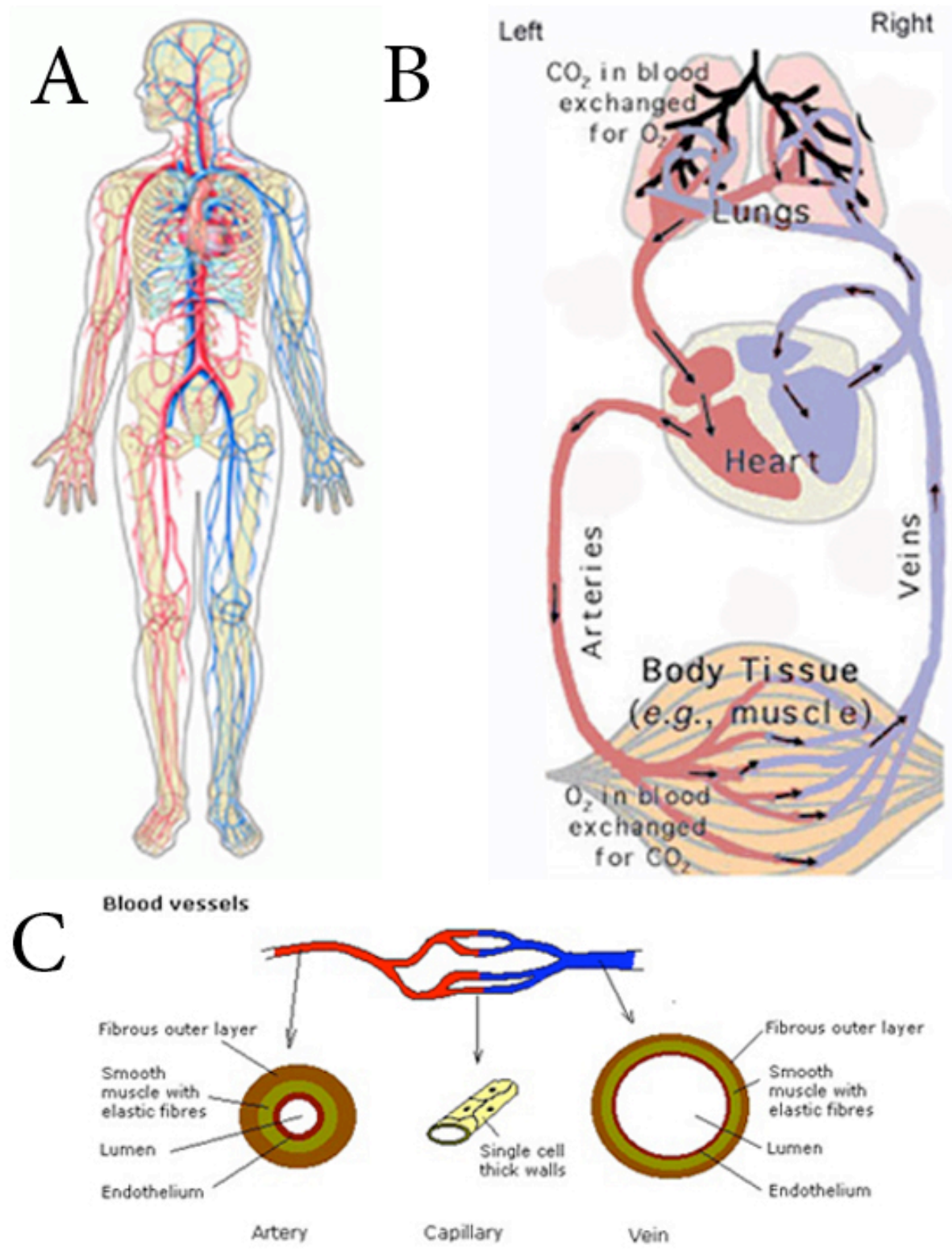

Figure 1. Circulatory system overview. A broad representation of the reach of the circulatory system (A) [8]. A simplistic diagram of the primary circulatory pathway in the human body (B) [9]. A basic illustration of arteriole, venule and capillary anatomical structure (C) ([10], permission by GNU Free documentation License). 


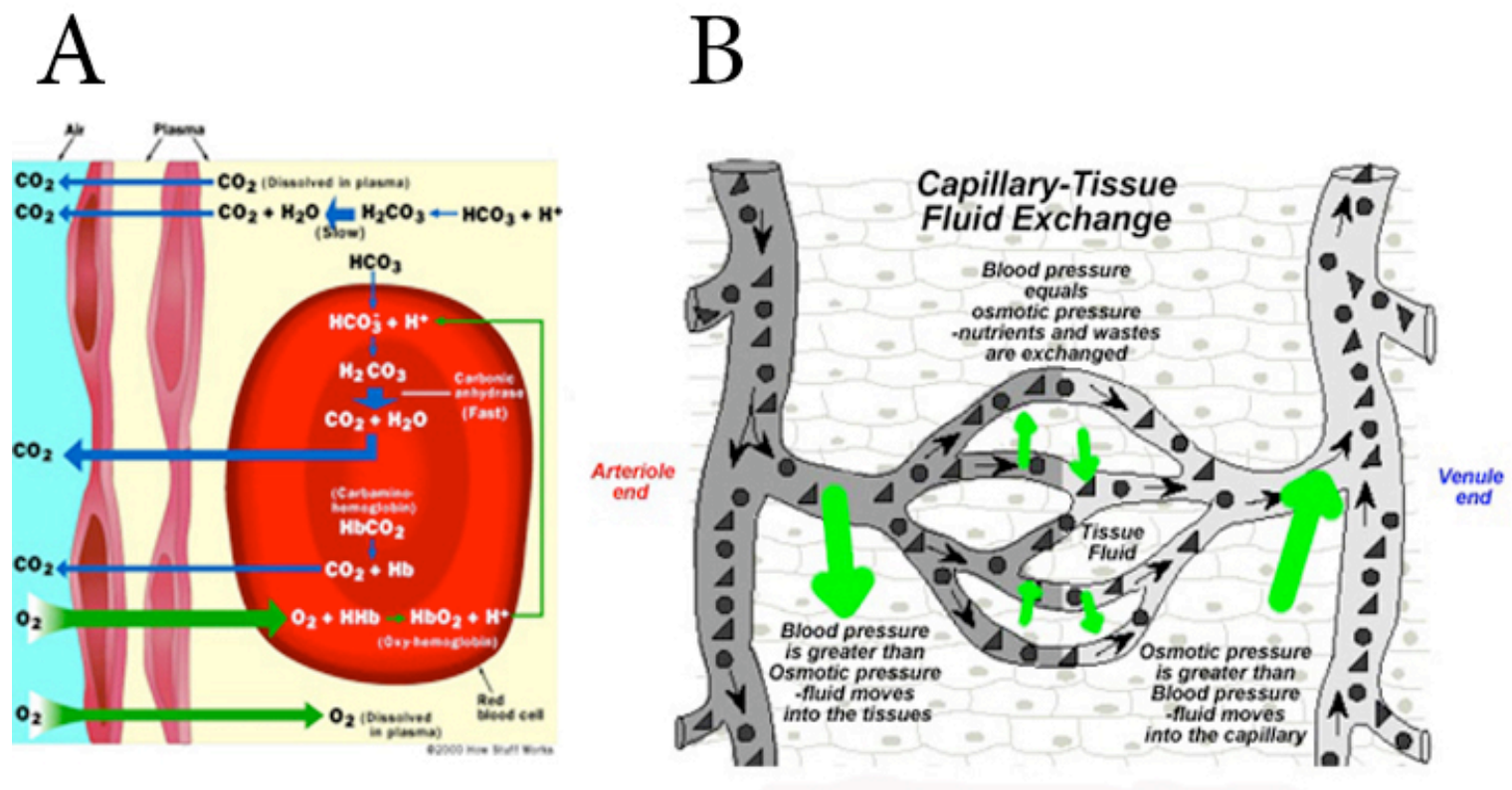

Figure 2. Nutrient exchange. Gaseous exchange in the lungs (A) ([11], Image provided courtesy of HowStuffWorks.com) . Fluid exchange in tissue capillaries (B) [12].

Circulatory system control and efficacy can be impaired through various mechanisms.

For example, edema can undermine nutrient exchange between the tissues and capillaries as the interstitial fluid volume expands, increasing the distance and delaying the speed of diffusion [13]. Vascular dysfunction can also be caused by narrowing or occluding of arteries, which induces insufficient blood delivery or ischemia [14]. This will be a key point, as this study will aim to identify the reason(s) for which ischemic insult abrogates normal vasodilation and vasoconstriction mechanisms. In human extremities this blood supply impairment (ischemia) can result in peripheral artery disease (PAD), which is of particular importance to this research [14]. Although this review of circulatory function is general in scope, it should be apparent that the circulatory system must negotiate varying metabolic demands within the body in an attentive and complex manner. 


\subsection{Peripheral Artery Disease}

As stated above, PAD is caused by insufficient blood flow to affected muscles and other tissues in the extremities. This reduction of blood flow to peripheral tissues is due to occlusion and narrowing of the vascular luminal space [15]. In most cases this obstruction is due to atherosclerosis (Figure 3), where extracellular cholesterol will build up along damaged/torn sections of arterial walls and be covered in a thin layer of fibrotic tissue [16]. This stenosis reduces volumetric blood flow via Poisseuille's Law. The resultant blood flow insufficiency (ischemia) is a detriment to the health of the extremities and must be ameliorated by vascular growth or by breaking apart or minimizing occlusions [15]. Further, while the resting blood supply is affected by this condition, vascular function (blood flow control) is also impaired (as previously mentioned). Furthermore, blood flow control is also important to patient health, as the earlier discussion would suggest [17]. Patients suffering from this condition commonly experience claudication (limb fatigue during activity) and pain in their feet while resting, specifically in the arch, ball or toes, and if the condition is not correctly treated, the symptoms may progress to include ulceration and tissue necrosis [18]. The latter symptoms of this disease are caused by total arterial occlusion in the extremity, a condition termed critical limb ischemia (CLI) [19]. The patient population affected by this disease (PAD) and these symptoms includes $10-25 \%$ of patients over the age of 55 , affecting 8 million people in the United States [16, 18]. Coronary artery disease, a related malady also caused by atherosclerosis, is the most prevalent form of heart disease and the leading cause of death for men and women in the United States [20]. In fact, PAD is a 
strong predictor, risk factor, and co-morbidity for CAD [21]. In one study $79 \%$ of patients with PAD also had CAD [21]. Total occlusion of coronary arteries results in a myocardial infarction or heart attack [20]. Furthermore, patients with CLI have an enhanced risk (300\% greater) to develop a myocardial infarction and stroke when compared to patients with only irregular claudication [22].
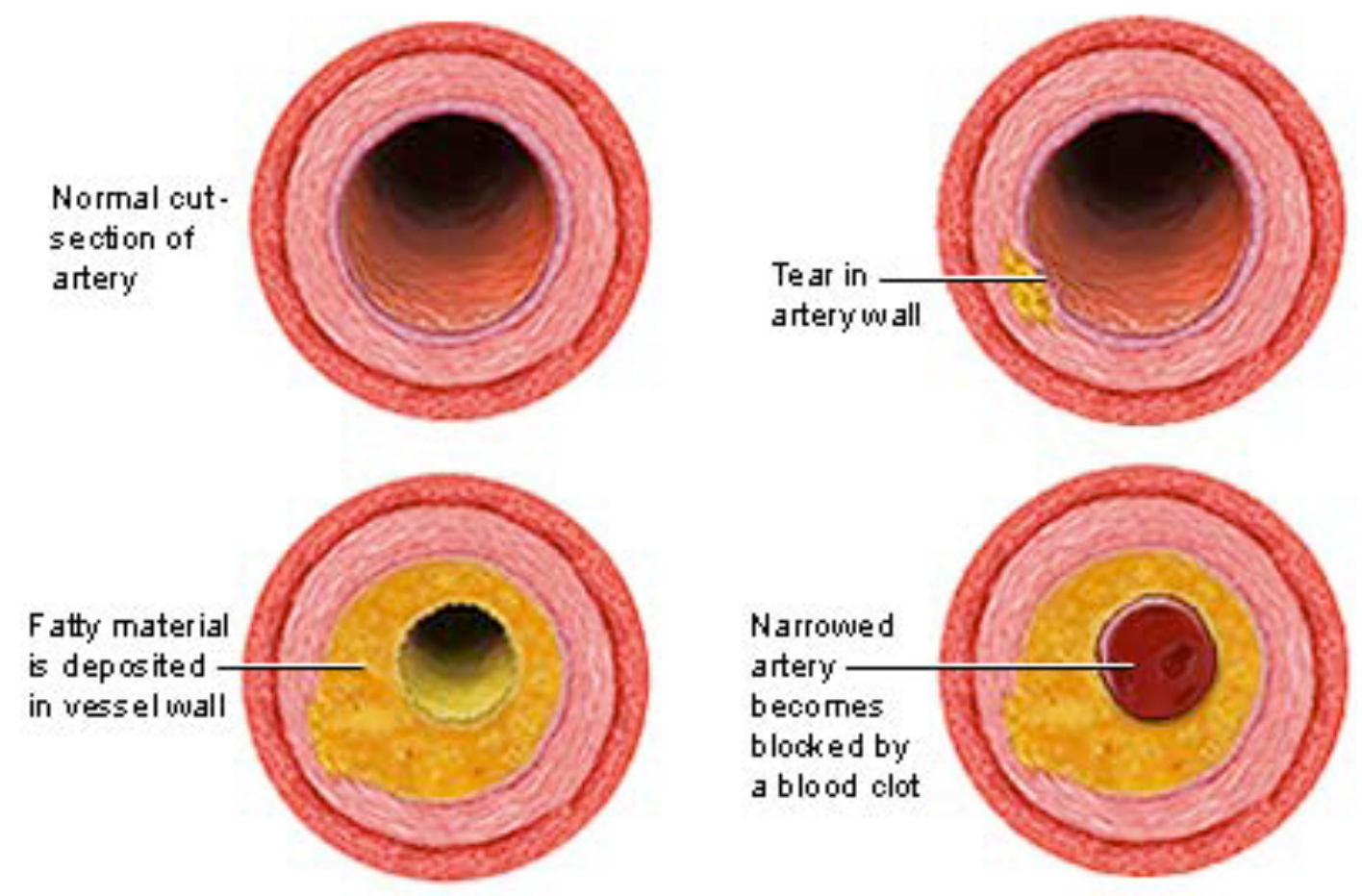

Figure 3. Atherosclerosis. The progression of chronic atherosclerosis in blood vessels to ischemia and complete arterial occlusion by blood clotting [23].

In patients presenting with early stage PAD and irregular claudication the current standard of care is conservative management with aspirin and statin drugs treatment [24]. Vascular growth promoting therapies are still being evaluated for potential benefit in 
alleviating ischemia and improving patient prognosis [25]. Should the disease progress to a more severe stage, as in CLI, surgical bypass, angioplasty and stent implantation are common treatment options [26]. If skeletal muscle perfusion and vascular function are not adequately ameliorated the disease may advance further and require limb amputation as gangrene and necrosis worsen [19]. This extreme intervention is often required when there has been multilevel total arterial occlusion and the previous interventions have failed [19]. The incidence of critical limb ischemia is approximately 500 to 1000 persons per million per year, with an even higher prevalence of cases among patients with diabetes [27].

To reduce the prevalence and minimize the disease progression through the development of better treatment options and therapeutic strategies it is imperative that disease mechanisms be further elucidated. Additionally, by expanding the knowledge of PAD pathogenesis, CAD pathology may be further understood given the overlapping etiology of the two conditions. Furthermore, it will be important to understand normal vascular growth and repair processes that work to ameliorate the associated ischemia (insufficient blood flow) and may play a role in ischemia-induced vascular dysfunction. In general, the process by which blood flow is salvaged is known as revascularization [28]. Revascularization is a broad term that can refer to surgical intervention, angiogenesis (sprouting of capillaries), or arteriogenesis (outward remodeling of arterioles) (two processes to be more vigorously discussed later) [28, 29]. As suggested vascular function is also important to tissue health following ischemic recovery. Developing an understanding of how angiogenesis, arteriogenesis and vascular function preservation 
relate (specifically, whether ischemia-induced vascular dysfunction is caused by either repair process) and lead to successful revascularization will be key to identifying efficacious treatments for PAD. A significant amount of information is already known about angiogenesis and arteriogenesis, knowledge that will aid analysis of this study of ischemia and revascularization.

\subsection{Mechanisms of VAscUlar GROWTH AND FunCtion}

\subsubsection{Arteriogenesis}

During ischemia, collateralization (arteriogenesis) is generally the most effective mechanism for blood flow recovery as it has the unique potential to fully compensate the blood flow lost from an occluded artery [30]. Collateralization describes the outward remodeling of linked arterioles into conduit arteries [31]. Chronic increases in shear stress have been demonstrated to induce arteriole growth and outward remodeling [32]. The mechanisms responsible for shear-stress-induced arteriogenesis, while not entirely understood have been elucidated in various vascular investigations. An enhancement of arterial shear stress due to an arterial occlusion, for example, causes endothelial swelling, inflammation and randomized orientation (as opposed to the usual orientation in the direction of blood flow) [30]. These intimal (inner layer of artery or endothelial layer) changes result in increased intercellular permeability and inflammation, as evidenced by red blood cells, platelets and plasma constituents seeping through the vessel wall [30]. Additionally, the shear stress stimulus activates endothelial cells (Figure 4) to release key arteriogenic cytokines [30]. Intracellular adhesion molecule (ICAM-1), vascular cell 
adhesion molecule (VCAM-1) and monocyte chemoattractant protein (MCP-1) are upregulated in endothelial cells following shear stress stimulation [33]. These soluble molecules promote monocyte migration and adhesion to the affected collateral vessels within $12 \mathrm{hrs}$ of arterial occlusion [30, 33].
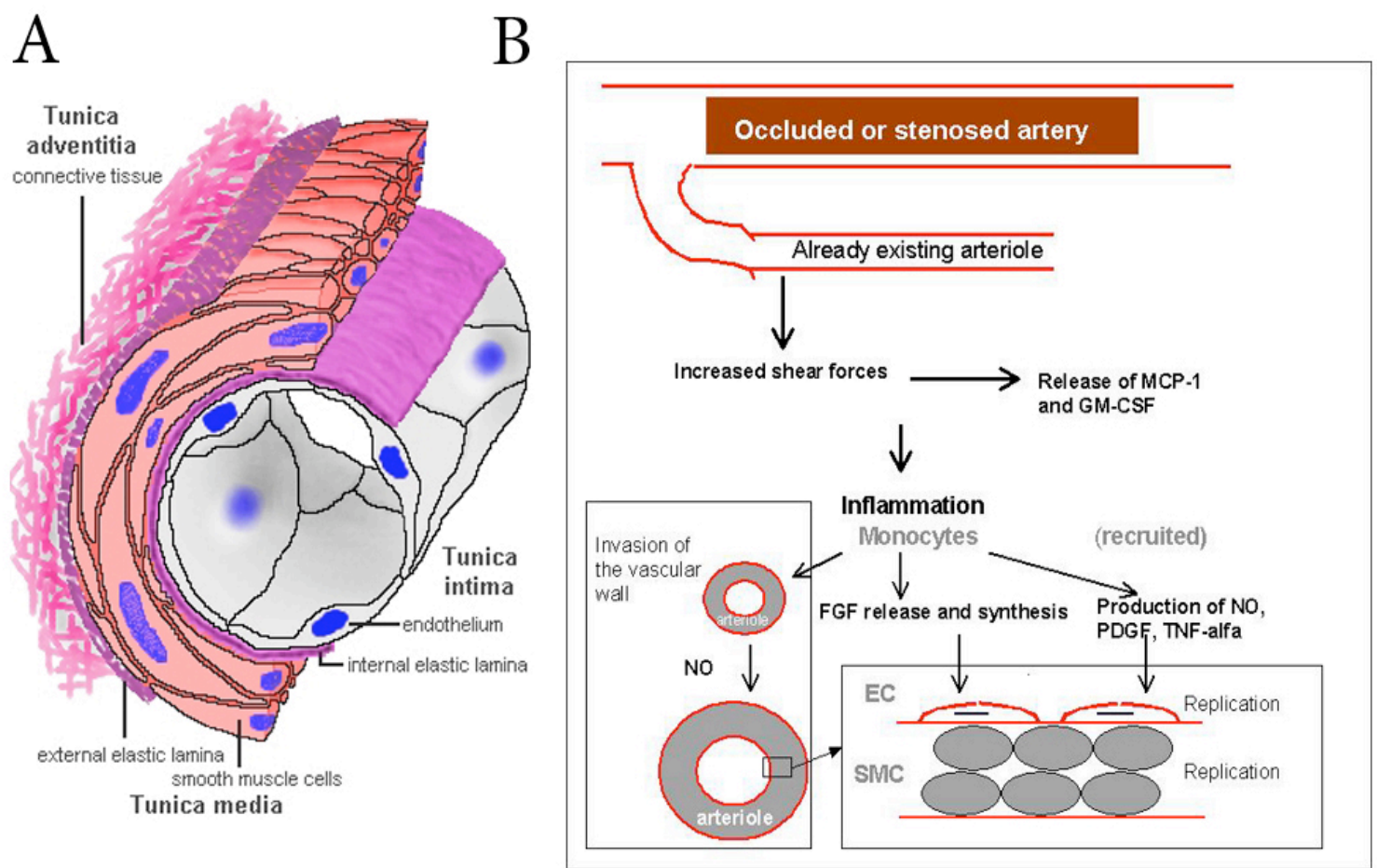

Figure 4. Arterial layers and outward remodeling mechanisms. The anatomical structure of an arteriole (A) [34]. Mechanisms underlying arteriogenesis (B) [35].

The monocytes and macrophages recruited to this vasculature site promote the previously described inflammatory response and release growth factors including basic fibroblast growth factor (bFGF) and tumor necrosis factor-alpha (TNF $\alpha$ ), which act to initiate cellular proliferation $[33,36]$. Specifically, endothelial cells in the intima and smooth muscle cells (SMCs) constituting the media of collateral arterioles begin to proliferate 
$22 \mathrm{hrs}$ following an arterial occlusion [37]. Further, SMCs proliferate to form a synthetically active (producing large amounts of growth promoting factors/proteins) neointima layer, which works to potentiate SMC growth and vessel remodeling [30, 38]. This layer is maturely developed in remodeled coronary arteries, but less developed in the periphery [30]. Another process vital for arterial remodeling is the proteolysis of the external and internal elastic lumina and basement membranes as well as apoptosis of SMCs, in order to expand the arterial lumen (equivalent to knocking down a wall to make a larger room) [30]. Macrophages and activated fibroblasts in the adventitia secrete matrix metalloproteinases (MMPs), which act to aid the remodeling process through proteolysis of the elastic lamina, which promotes migration of SMCs and arterial lumen expansion [30].

In general, SMC proliferation is the primary mechanism for arterial remodeling [30]. This SMC expansion is induced through increases in shear stress, which activate endothelialdependent pathways (previously described) and cause endothelial cell deformation [30]. Endothelial cell (EC) changes and the resultant EC release of various arteriogenic factors, following the specified deformation, are thought to be the primary mediators for remodeling. Nitric oxide (NO) and vascular endothelial growth factor (VEGF) have been implicated as promoters of collateralization likely induced by endothelial, shear-stressactivated signaling [39-41]. However, the arteriogenic sources and mechanisms for these factors have yet to be fully elucidated [30]. For example, endothelial nitric oxide synthase (eNOS) was long thought to be responsible for NO induced arteriogenisis when eNOS was observed to promote vasodilation during blood flow recovery, but was eventually 
demonstrated to be an ineffective mediator for collateral growth [42]. Still, multiple experiments have demonstrated that inhibition of $\mathrm{NO}$ is detrimental for collateral growth, suggesting that the key arteriogenic source for NO may be other NO donors such as inducible nitric oxide synthase (iNOS) [30, 40]. Much about arteriogenic mechanisms has been elucidated (as previously described), however, the role of NO or the specific mechanistic deviations from other repair processes have not been determined.

\subsubsection{Angiogenesis}

Although angiogenesis is not capable of renewing blood flow to the same degree as arteriogenesis, this distinct vascular growth mechanism has also been extensively researched for potential applications in PAD, cancer, age-related macular degeneration (AMD) and other vascular disorders [43]. Angiogenesis is described as capillary generation (Figure 5) and often occurs when tissues are subjected to a hypoxic environment (oxygen deficient) because of severe ischemia or other reasons. Newly formed capillaries function to supply blood to nutrient deficient (due to ischemia) tissues. The lack of oxygen in ischemic tissues activates various signaling pathways that upregulate key soluble factors required for endothelial cell migration, capillary sprouting and formation [44]. Angiogenesis inducing factors such as vascular endothelial growth factor (VEGF), placenta growth factor (PIGF) and NO have been identified through the use of transgenic and knockout mice [45, 46]. The most extensively examined factor, VEGF, has been shown to modulate endothelial cell function (protein/cytokine synthesis) and migration in the formation of new capillaries [47, 48]. Specifically, VEGF-C has been shown to not only induce the production of chemo-attractants to mediate endothelial 
cell migration, but also induces endothelial cells to release NO and increase vascular permeability to facilitate migration [49].

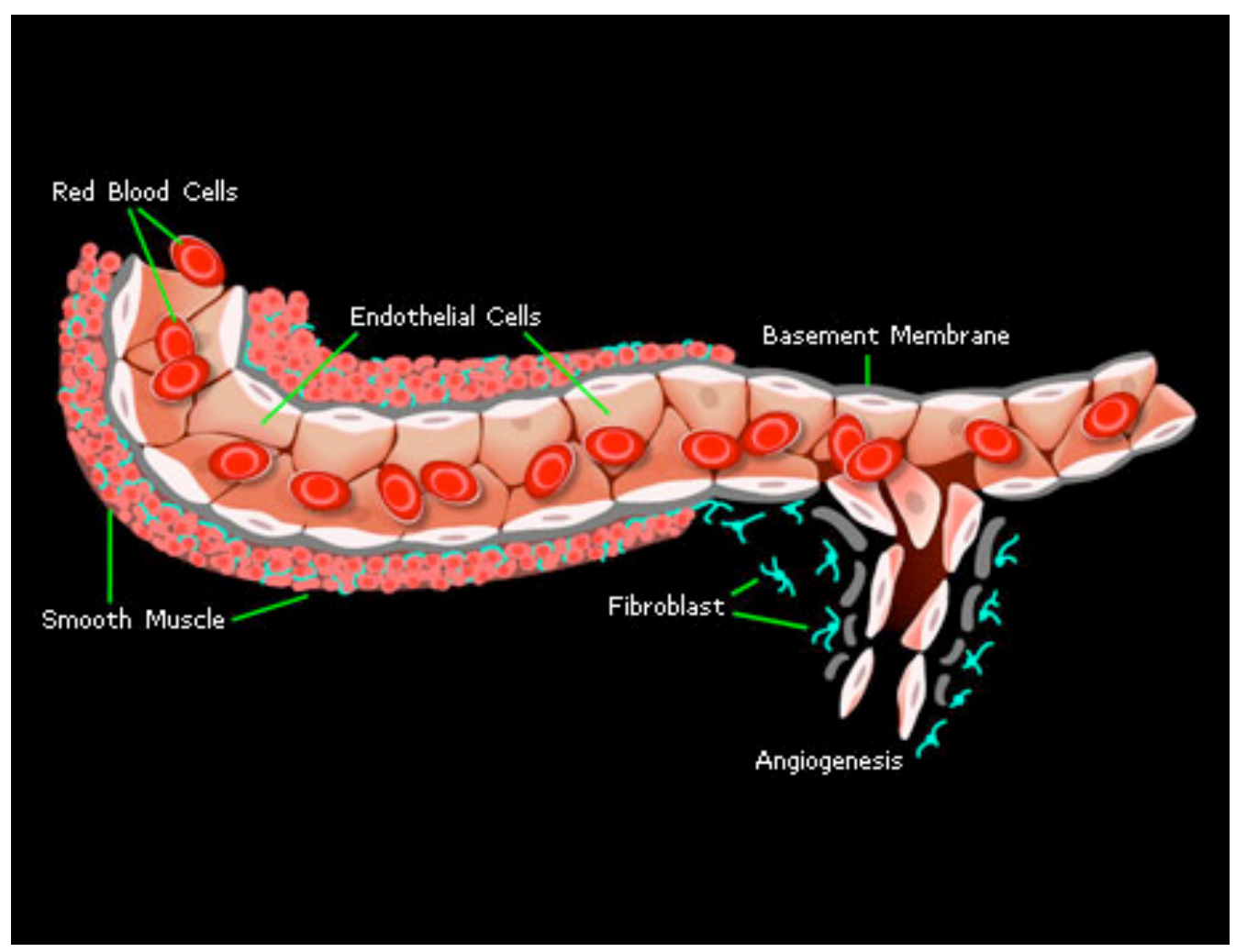

Figure 5. Angiogenesis of blood vessels [50].

There has been significant research done to identify angiogenic factors because these factors are also used by cancerous tumors to induce required blood perfusion. For this reason, the identification and targeting of these factors have been beneficial in slowing or ceasing tumor growth $[51,52]$. As discussed earlier, understanding angiogenesis is also important for the treatment of ischemia and peripheral artery disease, because inducing angiogenesis could facilitate the expansion of capillary networks and reduce diffusion distances thereby promoting nutrient exchange to hypoxic tissues. 


\subsubsection{Blood Flow Control Pathways}

While the aforementioned vascular growth mechanisms are critical for revascularization, recovery of vascular function is also important to insure the restoration of adequate tissue health. For example, the ability for remodeled vessels to vasodilate (luminal expansion) or vasoconstrict (luminal narrowing) according to downstream tissue demands is imperative to tissue function. As can be seen from Poiseuille's equation (Appendix A), these mechanisms can be used in tandem to effectively control blood flow (Figure 6). Specifically, in the case of ischemia, especially acute ischemia, vasodilation is utilized

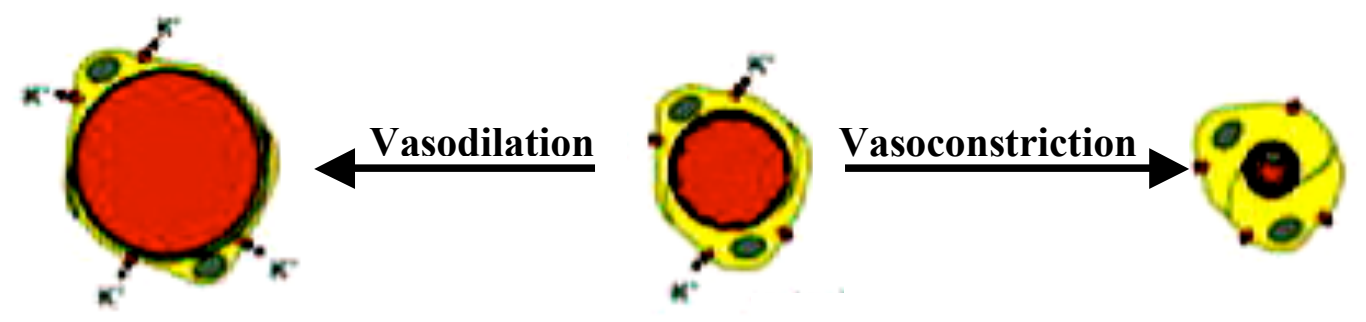

Figure 6. Arteriole blood flow control through vasodilation and vasoconstriction [53].

to return arterial blood flow to the level required by the tissue metabolism. For example, there are instances when arteries become occluded for a short time period and following the removal of this obstruction the downstream tissue responds through vasodilation to restore normoxia to tissues distal to the ischemic event, in a response known as reactive hyperemia [13]. Similarly, during exercise and increased skeletal muscle metabolism, arterioles will dilate to increase blood flow to exercising muscles in a process known as functional hyperemia [13]. 
Many factors that control arterial blood flow (i.e. during hyperemic events), such as various vasodilators and vasoconstrictors, have been identified through the use of various animal models. Nitric oxide (NO) is a significant vasodilator shown to play a key role in vasodilation as well as angiogenesis and arteriogenesis [46]. Additionally, acute increases in arterial shear stress due to blood flow has been shown to cause arterial vasodilation [5]. Moreover, arterial shear stress and vasodilators including acetylcholine and bradykinin can mediate dilation through the activation of eNOS to produce NO $[5,46]$.

Alternatively, various vasoconstrictors have also been identified, such as norepinephrine, vasopressin and angiotensin II [7]. These factors constitute some of the pathways for vascular diameter modulation. However, in conditions of chronic ischemia blood flow control mediated by vasodilators such as adenosine and bradykinin as well as vasoconstrictors such as norepinephrine is attenuated $[5,17]$. The mechanism by which these vaso-active agents fail during chronic ischemia would aid in our understanding and treatment of peripheral artery disease, because even if new treatments are able to promote vascular growth it remains imperative that these new or remodeled vessels be able to function according to the needs of skeletal muscle metabolism; proper vascular function is vital for adequate tissue function.

\subsubsection{Interrelation Between Vascular Growth and Function During Ischemic Repair}

To bring these concepts together let's quickly review repair progression following ischemia. After an ischemic insult (i.e. PAD) affected tissues normally respond to ameliorate insufficient blood flow by promoting vascular growth through arteriogenesis or angiogenesis, depending on their physiological environment (i.e. high shear stress or 
hypoxia). For reasons that we aim to discover, vascular function (ability for vasodilation/vasoconstriction) is impaired following ischemic repair [5, 17]. Certain arteriogenic or angiogenic physiological settings are expected to be the cause of this ischemia-induced vascular dysfunction. In this research we test the hypothesis that arteriogenic repair induces blood flow control impairment. To test this hypothesis we will utilize commonly used animal models of ischemia and revascularization.

\subsection{Mouse Models of Hindlimb Ischemia}

Animal models have often been used to analyze ischemic repair. One model that is compatible with both the project needs (in respect to ability to test our hypothesis) and facilities available (accommodations appropriate for animal housing, experimentation and survival), is a mouse model of hindlimb ischemia. In general, this model involves obstruction of hindlimb vasculature to cause insufficient blood flow and prompt an ischemic response. Within this animal model there are multiple surgical models (different sites or methods of arterial occlusion) with which we can utilize to induce distinct ischemic settings in the hindlimb.

To tease apart the potential reasons for vascular dysfunction it would be useful to use multiple surgical models for ischemia in which revascularization occurs under distinct physiological conditions. The previous studies, which observed impaired vasodilation to both metabolic and pharmacological stimuli were done using one rat model of hindlimb ischemia in which the iliac artery was ligated and vascular function was evaluated in the 
lower hindlimb $[5,17]$. Following ischemia-inducing surgery there are multiple factors that could influence vasculature dysfunction such as inflammation, oxygen delivery and average blood flow. In the previously referred to experiments involving iliac artery ligation, vascular function in the lower limb was evaluated and observed to be impaired under conditions of significant hypoxia, ischemia and inflammation $[5,17]$.

It remains unclear which specific factors influenced blood flow control impairment. So, by using two distinct mouse models of hindlimb ischemia (which create two distinct tissue environments) we may be able to identify which factors are correlated with the loss of appropriate vascular control. In one of the surgical models we will use, involving ligation of the femoral artery, it is anticipated that blood flow will be high, oxygen delivery will be normal and inflammation will be less severe in collateral arterioles than in the other model (involving resection of the femoral artery), where tissues are expected to be more hypoxic and ischemic (models will be more rigorously discussed later). In general, the idea is to examine vascular function of an arteriole "in series" and "out of series" of diverged blood flow following ischemia induced by arterial occlusion (Figure 7). To clarify, an arteriole "in series" with diverged blood flow experiences high shear stress, inducing outward remodeling/arteriogenesis, while an arteriole "out of series" experiences normal-low blood flow (as a major source of blood flow is cut off) and reduced collateralization (arteriogenesis). In another way, one model will examine blood flow control under a distinctly arteriogenic revascularization environment, while the other will evaluate the same vascular response in non-arteriogenic promoting conditions. To reiterate, the creation of distinct ischemic settings through the use of two surgical 
models should help to tease apart the underlying (potentially repair-related) causes of ischemia-induced vascular dysfunction. By identifying these causes, this study hopes pinpoint potential therapeutic targets for patients with PAD experiencing inadequate blood flow control and claudication.
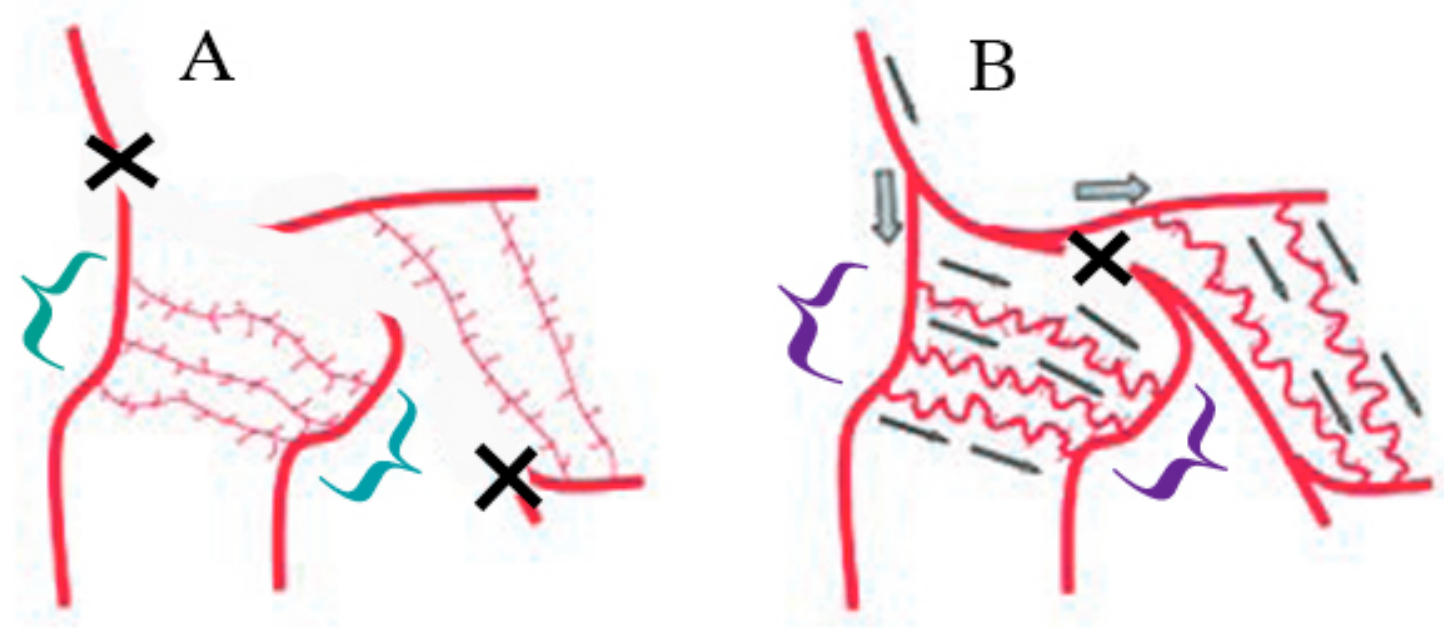

Figure 7. Generalized juxtaposition of surgical models. In general the idea is to examine the revascularization conditions of conduit arteries (brackets, gracilis arteries in the hindlimb) following distinct occlusion scenarios of the main blood supplying vessel (top vessel, femoral artery in the hindlimb). One model isolates arteries "out of series" (within green brackets) with diverged blood flow following ischemia inducing arterial occlusion (designated by crosses) (A). Another model examines arteries "in series" (within purple brackets) with diverged blood flow following ischemia inducing arterial occlusion (B) (adapted from [18]).

\subsubsection{Distinctions Between Animal Ischemia Models and PAD Etiology}

The mouse models used were not meant to model the exact etiology of PAD, but to model one aspect of the disease pathology, specifically chronic limb ischemia. Modeling and examining vascular repair/function in the context of ischemia should prove useful in 
the development of treatments for PAD, as insufficient blood flow is a significant source for poor tissue health in patients suffering from this disease. Even more, these models provide a simplified ischemic setting to examine dysfunction development. It is imperative to describe the differences between the mouse hindlimb ischemia models and PAD such that model limitations are elucidated and understood.

As discussed earlier, the models aim to parallel ischemic conditions in the extremities of patients with PAD. However, the causes for the ischemic conditions in the models are significantly different than those inducing ischemia in PAD. Ischemia with PAD is a result of atherosclerosis, which occurs chronically, via gradual build-up of plaque and narrowing of the arteries over many years [15]. Complete occlusion of arteries often results in limb necrosis without proper treatment (10-40\% have primary amputations and there is a mortality rate of $40-70 \%$ in 5 years) [54]. In contrast, both of the mouse arterial occlusion models do not occur gradually, as the femoral artery is completely occluded within the span of a few surgical motions.

Further, as stated above, complete arterial occlusion in the human disease likely results in limb necrosis with conservative (non-surgical) treatment (within 1 year $95 \%$ of surviving patients with tissue loss, not treated surgically require amputation) based on the population of patients that present in the hospital (there is likely an uncharacterized subset of patients that do not present in a hospital and are able to revascularize), whereas in the mouse, hindlimb revascularization occurs and the mouse is able to recover over a period of 2-4 weeks without any intervention or treatment [42]. This disparity is likely 
exaggerated by the presence of co-morbidities (i.e. heart disease, obesity, etc.) in the human condition. The gradual arterial narrowing of atherosclerosis also results in a slower impairment of blood flow and progression of hypoxia, which may result in a different timeline for vascular repair. Additionally, atherosclerosis is often found at multiple vascular sites in the circulatory system of the extremities [15]. However, the surgical mouse models result in arterial occlusion at one point in the femoral artery. Hence, while both PAD and the hindlimb ischemia models induce ischemia in the extremities (hindlimb), the mechanisms that each insult utilizes to affect that change are distinct. Furthermore, it is imperative to understand those differences such that it is possible to place any knowledge gained about vascular repair under ischemic conditions into the correct context. Knowing this, the mouse ischemia models can still provide important information in regards to vascular repair, as we expect to examine the process of revascularization and how this repair process may influence recovery of vascular function following ischemic insult.

The two mouse models of hindlimb ischemia focus on the medial aspect of the upper hindlimb under various methods of femoral artery occlusion. Revascularization following each occlusion model was evaluated in the vasculature of the upper hindlimb, specifically the muscular branch (for an overview of the hindlimb vascular structure prior to the upcoming more focused examination of each model see Figure 8). 


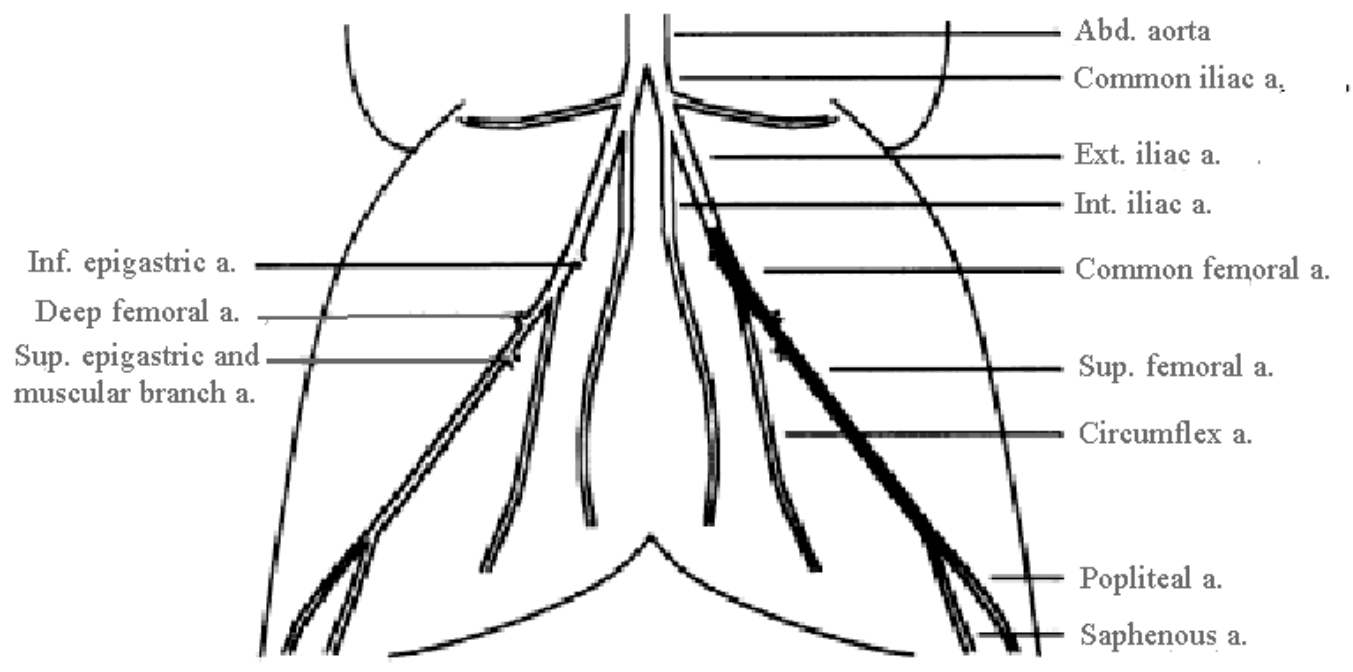

Figure 8. Arterial map of rabbit hindlimb (rats, mice and other animals have analogous structures) (adapted from [48]).

\subsubsection{Femoral Artery Ligation Model}

The first hindlimb ischemia model used to examine the revascularization of arteries feeding the gracilis muscle involved the ligation of the femoral artery downstream to the muscular branch and deep femoral arteries (Figure 8,9). This arterial occlusion model induces both blood flow changes and repair processes that we hope to relate to PAD. First, there will be a divergence of blood flow that previously traveled through the femoral artery and to the saphenous artery $[42,55]$. Instead, this blood will not be able to flow through the femoral artery and will flow through the deep femoral and muscular branch arteries, in addition to alternative conduits in the deep hindlimb muscle $[42,55]$. 


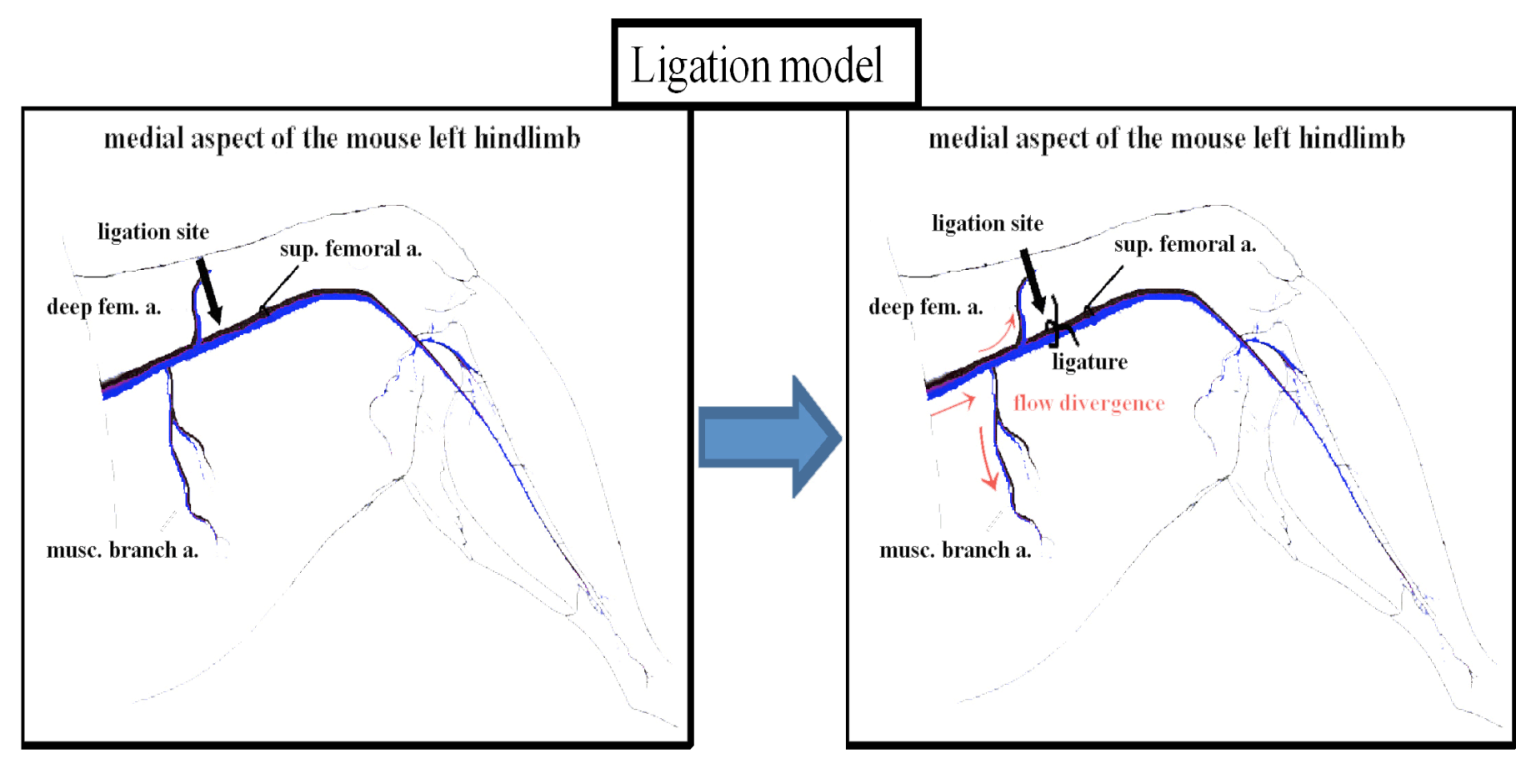

Figure 9. Diagram of the femoral artery ligation model. The red arrows show blood flow divergence following the surgery.

This arterial insult affects different areas of the mouse hindlimb in distinct ways.

Perfusion of the lower hindlimb or the calf is significantly decreased (Figure 10), because following femoral artery ligation there are essentially no more large arteries to feed this part of the extremity. Due to this severe ischemia, oxygen and other nutrients are not easily transported to the lower limb and the affected tissues experience hypoxia and inflammation, environmental factors that promote angiogenesis [42, 55]. Alternatively, the upper hindlimb likely experiences mild hypoxia to normoxia as blood flow is generally increased, because of the blood flow being directed away from the femoral occlusion (see Figures 9 and 10). An increase in wall shear stress due to the elevated blood flow will have both acute and chronic consequences. A sharp increase of shear wall stress will result in arterial vasodilation mediated through the activation of endothelial nitric oxide synthase (eNOS) (to produce NO) [5].Hence, an acute outcome will be 
vasodilation due to blood flow divergence and increased shear wall stress. As this occlusion will be permanent, chronic increases in wall shear stress will also be relevant to physiological recovery from the ligation surgery. As previously stated long lasting increases in shear wall stress induce arteriogenesis (refer back to Figure 5) [30, 32, 33]. Thus, arteries perfusing muscles of the upper hindlimb (i.e. gracilis muscle) will outwardly remodel (Figure 11) and act as the primary mediators for blood flow recovery $[55,56]$. This arteriogenic response as well as the relevant ligation-induced conditions (high shear stress, normoxia and modest inflammation) will be important to recall and contrast with the revascularization environment in the resection model. This juxtaposition will be important to illuminate the physiological causes for the impairment of vascular function following chronic ischemia and eventually improve our understanding of PAD. 

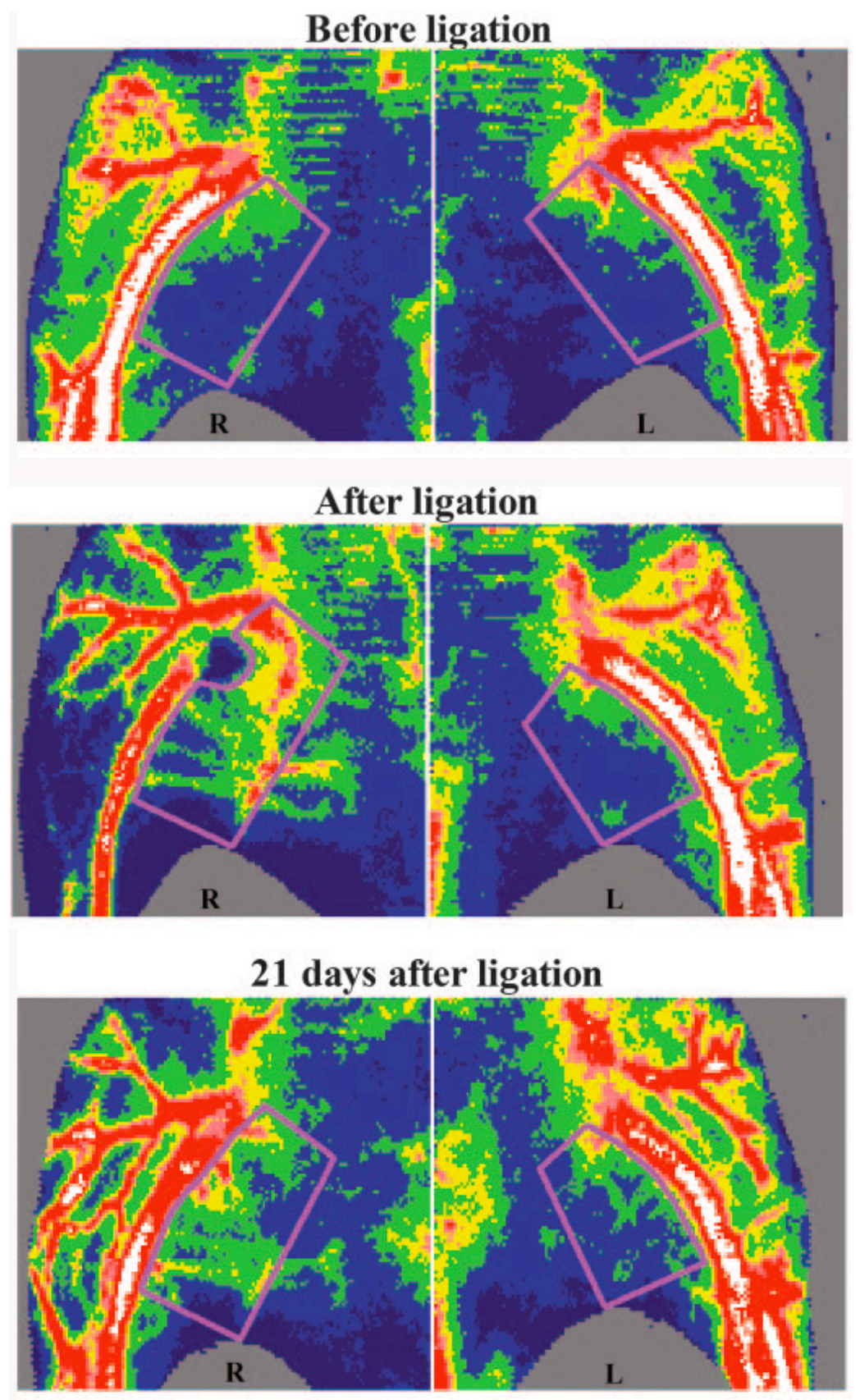

Figure 10. Hindlimb perfusion changes following femoral artery ligation. Scanning Laser-Doppler perfusion images of blood flow changes in the right ischemic limb immediately following and 3 weeks following femoral artery ligation compared to the perfusion changes in the nonischemic left limb [55]. 


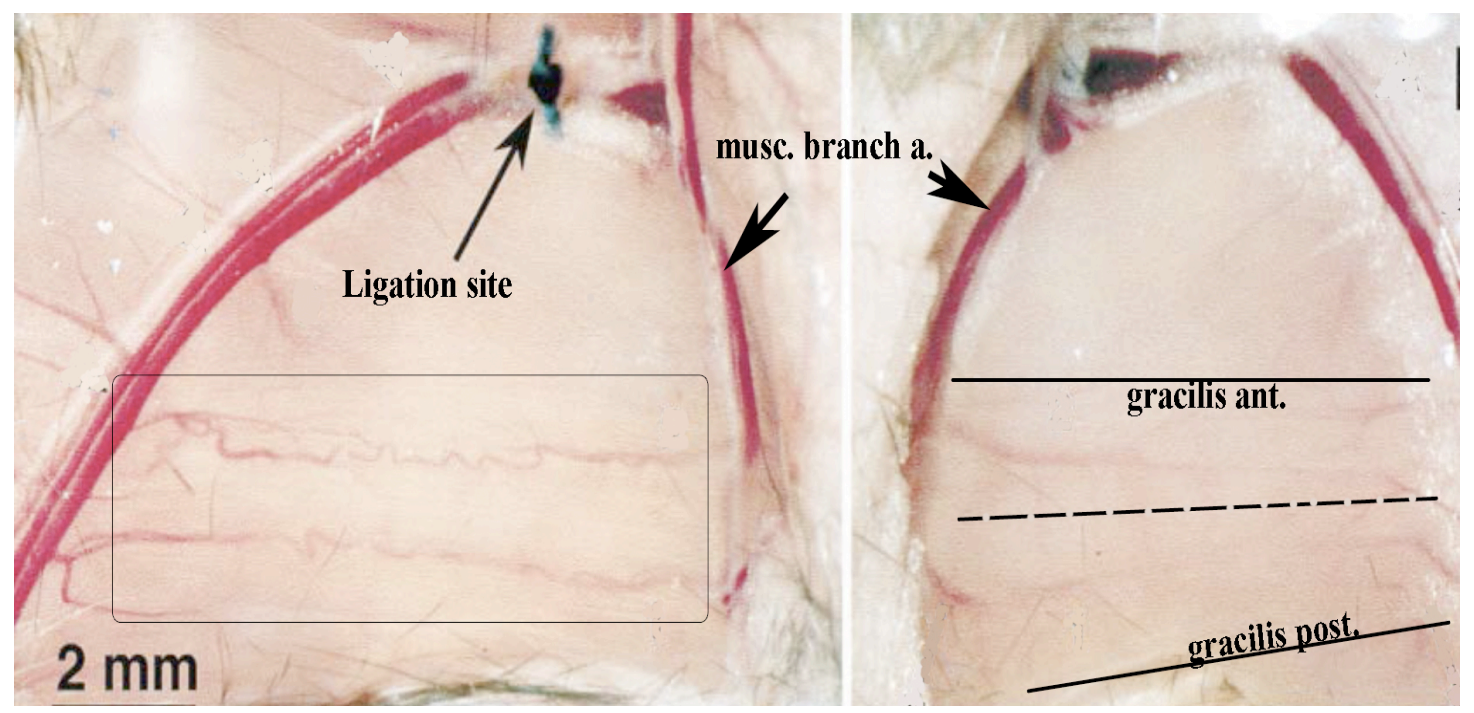

Figure 11. Arteriogenesis following femoral artery ligation. Collateralization of gracilis arterioles in the hindlimb (rectangular box) following ligation on left and a picture of the non-ischemic hindlimb on the right (adapted from [32]).

\subsubsection{Femoral Artery Resection Model}

As mentioned, a resection model of hindlimb ischemia will also be used in order to examine distinct revascularization conditions. In this resection model the femoral artery is removed from just proximal to the muscular branch artery to half way between the knee and the heel or soon after the saphenous artery branch point (Figure 12). Branching arteries are either cut or cauterized as the majority of the superficial femoral artery is removed. Based on previous research, this model impedes the majority of blood flow to the hindlimb, as blood flow can no longer be diverged to the muscular branch or deep femoral arteries directly from the femoral artery [44]. As in the femoral artery ligation model the lower and upper hindlimb experience and react to distinct tissue environmental conditions. In this model the tissues in the lower leg are again subjected to a hypoxic and ischemic environment similar to the femoral artery ligation model [44]. Further, 15 days 
following both the femoral artery ligation and resection models the perfusion of the lower ischemic hindlimb is approximately $60 \%$ of the nonischemic limb $[44,55]$. As a result, angiogenesis is significantly increased in the lower hindlimb of this model as well, since it has been demonstrated that hypoxia is a crucial driving factor for angiogenesis [57]. In the upper hindlimb, following resection of the femoral artery, blood flow will primarily come from other, smaller feed arteries, such as the medial circumflex, which will supply the muscular branch artery and gracilis microcirculation with needed nutrients [58].

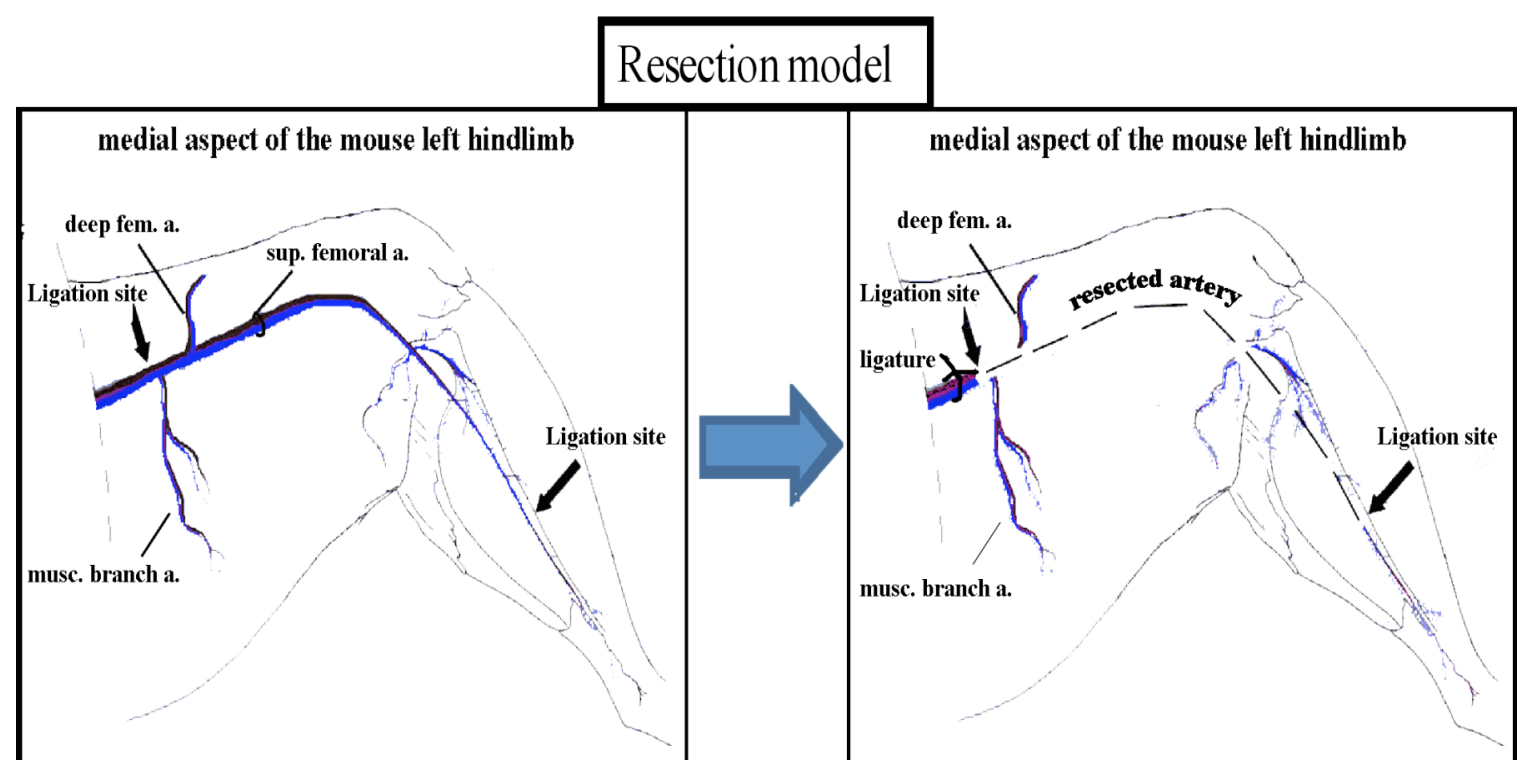

Figure 12. A diagram of the femoral artery resection model for hindlimb ischemia (the superficial femoral artery section between each ligation site was removed and one ligature was left in).

Following surgery, arteriogenesis will occur in the medial aspect of the upper hindlimb. However, it is anticipated that this remodeling will occur to a lesser extent in the gracilis muscles when compared to the ligation model and furthermore repair will occur under 
different conditions. For example, blood flow and thus shear stress in the arteries perfusing the upper hindlimb (specifically the gracilis muscles) should be reduced when compared to the same vessels following arterial ligation. Further, tissues of the upper hindlimb should be exposed to greater hypoxia in the femoral resection model. Relatively more severe hypoxia in the upper hindlimb following femoral artery resection should be expected based on the extensive arterial excision and insult to hindlimb perfusion when compared to the blood flow increase in the ligation model, as blood is redirected to the gracilis muscles directly through the muscular branch (impossible following resection) (Figure 11). A simple ligation of the femoral artery proximal to the muscular branch artery was not used due to the concern that blood flow through the muscular branch may be salvaged (inhibiting the contrast between models which is vital for comparison) by other feed arteries (the gracilis arcade), a consequence that is hindered by thorough excision of the majority of the femoral artery.

Separately, this model is also different, in that the extensiveness of the surgery should require a higher degree of invasive dissection, as the artery vein pair must be dissected from muscle, nerve and other blood vessels along a longer length. Given this and the more severe hypoxic and ischemic insult, it is expected that this surgery should induce more significant and ubiquitous inflammation of the hindlimb, promoting angiogenic revascularization. The fact that the resection model has stark distinctions in ischemic tissue conditions allows for the examination of vascular function following unique repair processes (predominantly arteriogenic vs. non-arteriogenic revascularization). 
Using both the femoral artery ligation and resection models, the aim of this study will be to examine the role arteriogenic revascularization processes/conditions (especially shear stress) may play in blood flow control impairment following chronic ischemia. The ultimate goal is to identify the mechanisms for vascular dysfunction following ischemia and utilize that knowledge to treat similar blood flow control impairments in PAD patients. The development of new technologies is aiding that aim. Unique microscopes that can evaluate blood flow in different ways as well as sensors to detect concentrations of key soluble factors such as NO and oxygen are helping to progress our understanding of revascularization mechanisms. In this project a novel video microscope with the capability to measure vasodilation in large arterioles and blood flow through complex microcirculation was used to evaluate vascular growth and vascular function following chronic hindlimb ischemia.

\subsection{SideSTREAM DARK FIELD IMAGING}

Sidestream dark field (SDF) imaging technology was used to asses the vascular repair process. This technology was developed following the advent of Orthogonal Polarization Spectral (OPS) imaging (Figure 14) [59]. Although analysis of microcirculatory function both clinically and experimentally is useful, investigation of the microcirculation was limited by the size and narrow scope of previous devices (prior to OPS) [59]. However, with the development of OPS and SDF imaging technology the size of vascular imaging devices has shrunk while the capabilities have expanded [59]. In SDF imaging, photons are emitted at a wavelength that is absorbed by hemoglobin molecules [59]. Meanwhile, 
photons not absorbed by hemoglobin scatter and reflect back to a detection lens (Figure 13) [59]. Light detected in this fashion appears light on the video, while blood vessels, which contain hemoglobin appear dark [59]. This creates a contrast, which allows for the distinction and visualization of red blood cell (hemoglobin is a primary constituent) carrying vessels (veins and arteries) [59]. Not only can these vessels be visualized, but blood flow can also be measured in many of the vessels utilizing computer software [59]. Clinically, this technology along with OPS imaging has seen increased use, as it has become a useful diagnostic tool for various diseases $[60,61]$. For our purposes, the SDF imaging microscope was used to evaluate vascular growth and function following revascularization of the muscular branch artery (which feeds the gracilis muscles) in vivo, by measuring changes in arterial diameter.

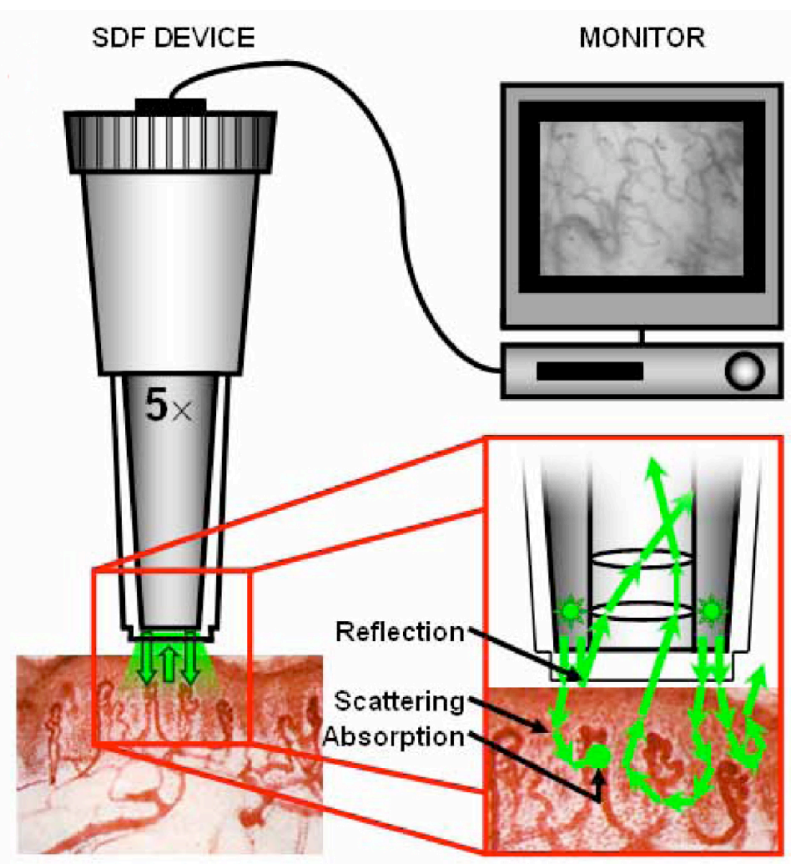

Figure 13. Diagram of SDF imaging mechanism. The SDF video microscope with and outer illuminating ring and a separate inner lens system. The microscope excites hemoglobin, absorbs emitted photons and a computer converts that information to a digital video (adapted from [59]). 


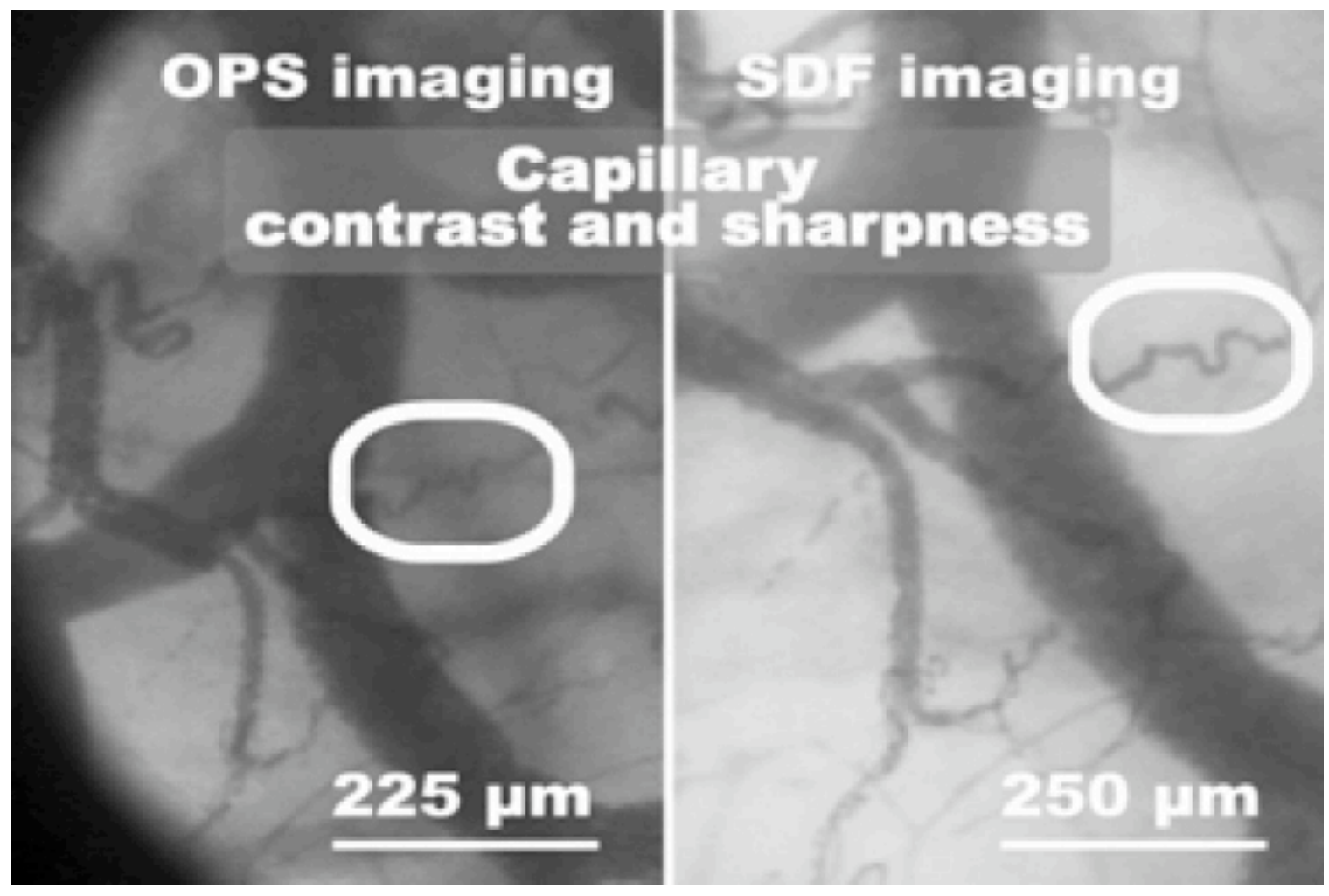

Figure 14. Digital video data examples from OPS and SDF imaging (adapted from [62]).

\subsection{OBJeCTIVES AND HyPOTHESES}

Experimentally, this study aims to examine collateral artery function following revascularization under arteriogenic and non-arteriogenic-inducing ischemic conditions. It is expected that this investigation will direct future research to the likely physiological factors (i.e. hypoxia, inflammation, etc.) that influence vascular function impairment. More specifically, there were three primary objectives that were set to direct this research to that ultimate goal: 
1. Establish a consistent protocol for the SDF imaging intravital microscope to examine muscular branch arterial changes during functional hyperemia induced by gracilis stimulation. The SDF microscope had not been used to image mouse vasculature in this way. Thus, there was a significant amount of troubleshooting early in the experiment.

2. Examine (using the established SDF imaging protocol) collateralization of the muscular branch artery two weeks after ischemic surgeries. This objective was established to validate a generally accepted concept based on previous research: femoral artery occlusion induces arteriogenesis in the upper hindlimb [55]. However, it would also be interesting to examine how collateralization of the muscular branch varies between the resection and ligation models.

3. Examine vascular functional hyperemia following arteriogenic and nonarteriogenic ischemic insult. The ultimate objective of this research, with the aim to direct future research.

It was initially hypothesized that the ligation model would induce vascular dysfunction compared to both the non-ischemic and resected limbs due to elevated arterial shear-wall stress (in the ligation model). 


\section{METHODS}

\subsection{HOUSING FOR MiCE}

Male C57/B6 mice were housed in the Cal Poly vivarium, with four mice to a microisolator cage. The room was maintained at $73 \pm 5.0^{\circ} \mathrm{F}$ and lights were on 12 hour light:dark cycles. Cages were changed at least once a week. Mice were provided food and water ad libitum in addition to the following enrichments: mouse house, nesting material, and plastic tube. During cage changes, lab members donned isolation outfits to preserve aseptic conditions. Further, mice were monitored on a daily basis, during which their health and any irregularities were recorded and/or addressed as necessary (see Appendix B).

\subsection{Vascular Function Experiments on Mice Not Surgically Altered}

Sidestream Dark Field imaging was used to study vascular function of the muscular branch in the mouse hindlimb (see Appendix $\mathrm{C}$ for experimental checklist). Experiments were performed on C57/B6 mice using isoflurane anesthesia. The mice were kept at a temperature of $35 \pm 1.0^{\circ} \mathrm{C}$ using a rectal-temperature controlled heating pad (Temperature Controller TR-200, FST). An initial incision was made on the medial aspect of the hindlimb, directly above the gracilis muscles. This incision was then widened to expose most of the medial aspect of the thigh, from the abdominal wall to approximately the insertion of the gracilis muscles into the tibia. Phosphate Buffered Saline (PBS) solution 
was applied to the exposed tissue to prevent desiccation of the skeletal muscles and vasculature.. Following the exposure of the medial aspect of the thigh, the fat pad perfused by the epigastric artery was blunt dissected posterior to the epigastric branch. This fat pad was then bisected at this dissection point using a heat cautery (Bovie, Aaron Medical). The two halves of the fat pad along with the abdominal wall were retracted to increase exposure of the muscular branch. The obturator nerve, which innervates the gracilis muscles, was identified and approximately $1-3 \mathrm{~mm}$ of its length was dissected proximal to its insertion into the gracilis muscles. The positive electrode was then inserted into a flap of skin near the knee to function as the ground (Figure 15). With the assistance of the $\# 5 / 45$ forceps the negative electrode was then inserted under the dissected aspect of the obturator nerve (Figure 16). The use of PBS solution was then replaced with mineral oil.

Mineral oil was used for several reasons. First, mineral oil does not permit oxygen exchange between the exposed hindlimb and outside air, unlike PBS. This is important, because if oxygen is allowed to be exchanged with the environment then it lowers that oxygen that the muscles demand from the circulatory system. Thus, arteries such as the muscular branch will likely be abnormally constricted because there is less oxygen delivery demanded from them. Plastic wrap was also considered, because it could also block oxygen exchange with the environment, however it was difficult to keep the wrap in place and adjust the SDF intravital microscope such that it was in an optimal position to visualize the muscular branch. 

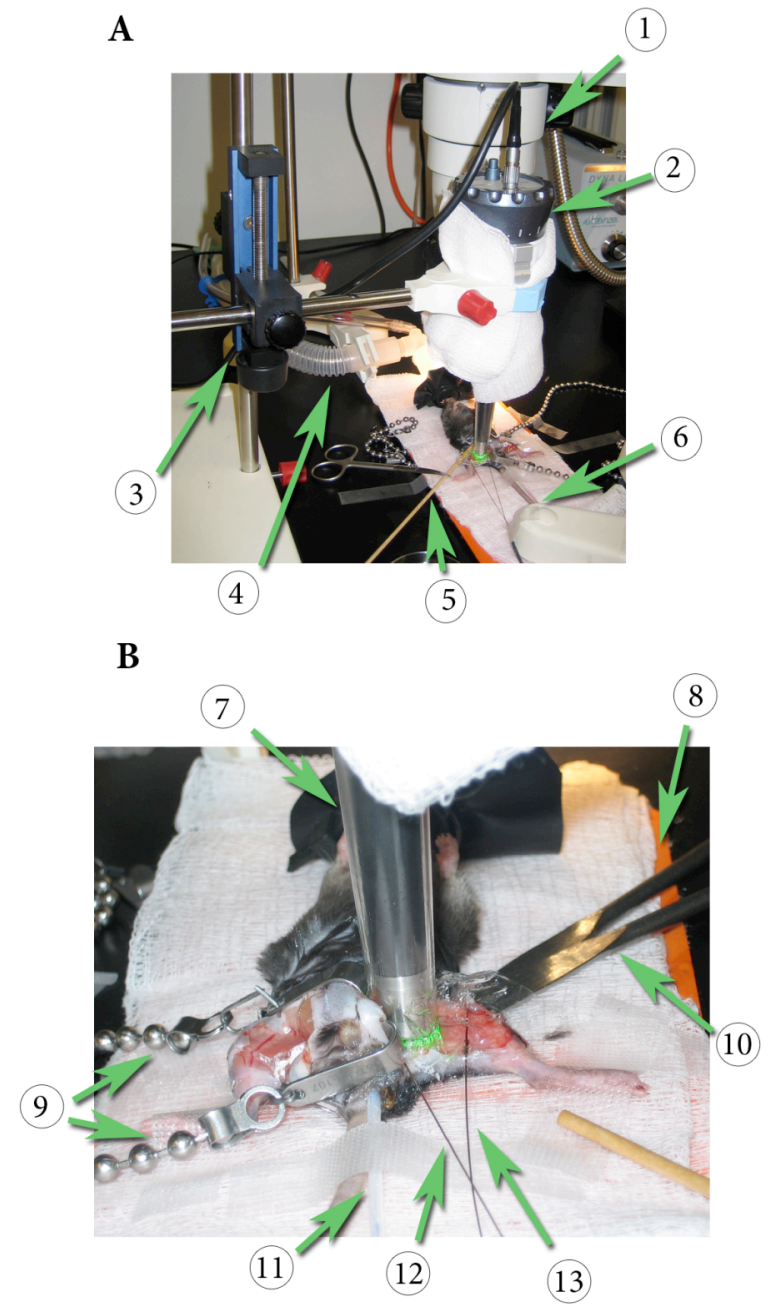

Figure 15. SDF imaging experimental setup. In the upper picture (A) the microscope used for dissection (1) (SMZ800 Zoom Stereo Microscope, Nikon) and SDF video microscope (2, also location of image focus and light control) are on either side of the animal to alleviate space issues such that they only need to rotate to switch positions. A chemistry stand with vertical fine adjustment (3) and clamp holding oxygen and anesthesia delivering tubes (4) aid experimental control. In the bottom picture (B) retractors (9) help to expose the muscular branch for visualization by the MicroScan probe (7), the heating pad (8) and rectal thermometer (11) maintain thermostasis, while improvisational instruments are used to raise (10) and lower (5) the hindlimb to be in the same horizontal plane as the probe. Finally, during stimulation, electrodes are used to stimulate gracilis contraction, such that the positive electrode (13) is inserted into a flap of skin and the negative electrode (12) is placed under the dissected aspect of the obturator nerve, with a chemistry clamp (6) used to adjust its height so it does not put too much tension on the obturator nerve and rip it. 


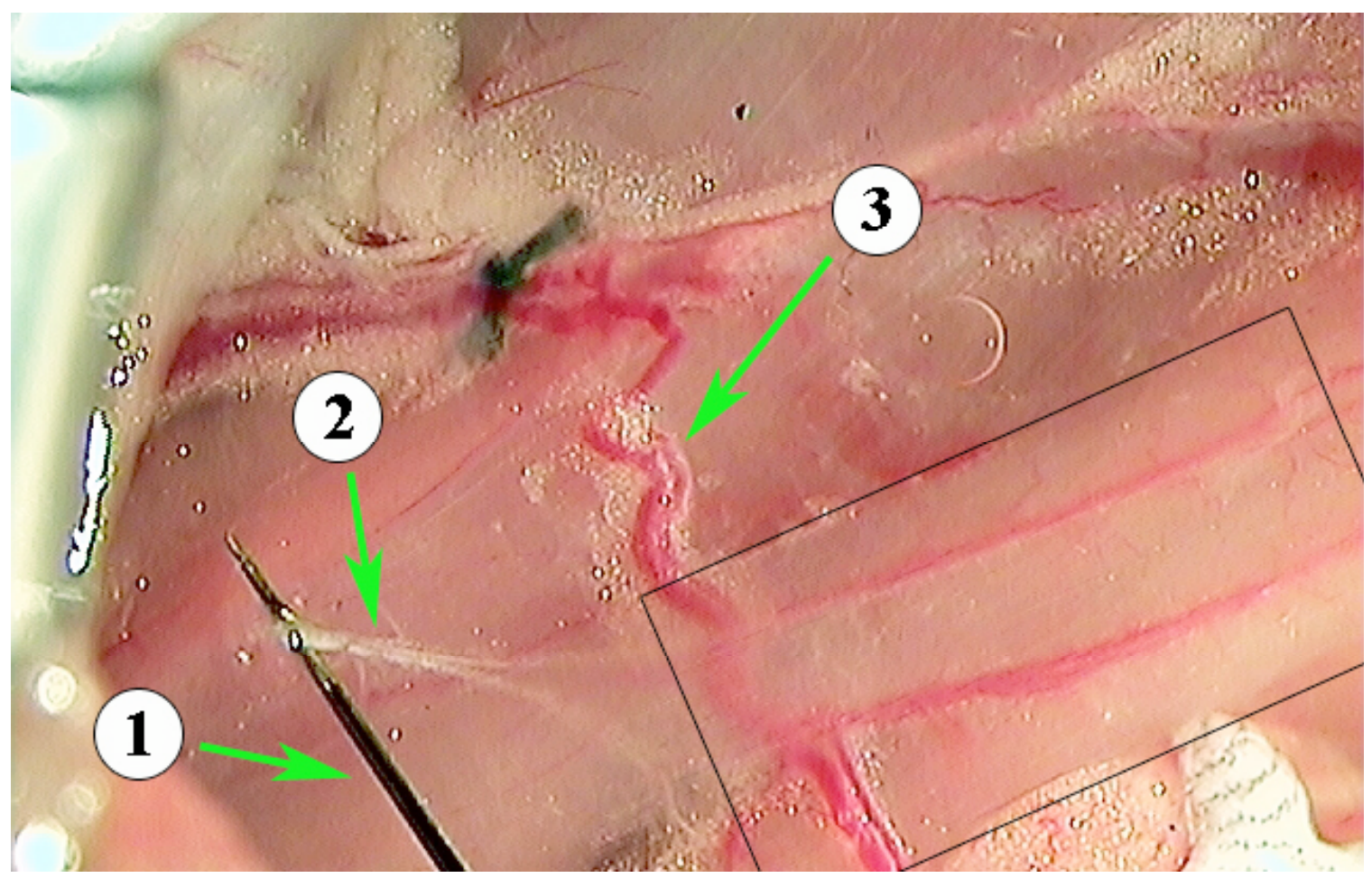

Figure 16. Placement of negative electrode. The negative electrode (1) was placed under the dissected portion of the obturator nerve (2), so that the SDF video microscope could image the muscular branch artery (3) following gracilis muscle (inside rectangular box) contraction.

The SDF intravital video microscope (MicroScan, Microvision Medical), attached to a chemistry clamp stand was then moved into an initial position to adequately image the muscular branch artery (Figure 15). This position was then finely adjusted while also adjusting the hindlimb to position it in the horizontal plane. Regions of the exposed muscles where the probe was not present were then covered with plastic wrap to reduce runoff of the oil. The hindlimb was then allowed to recover from the dissection and associated insults for 30 minutes. Following this thirty-minute rest/recovery period, a 10 second video using the inravital video microscope was taken. Subsequently, the obturator nerve was stimulated to contract the gracilis muscles at $8 \mathrm{~Hz}, 4 \mathrm{~Hz}$, or $2 \mathrm{~Hz}$, at a $500 \mu \mathrm{s}$ 
square pulse duration and 1.0mA pulse amplitude for 3 minutes. Immediately after stimulation another 10 -second video was recorded. Following stimulation the hindlimb was allowed to rest for 6 minutes such that the muscular branch artery was allowed to regain resting vascular tone. The stimulation and recovery process was repeated for each of the three frequencies. Frequency order was randomized for each mouse. Following all stimulations the hindlimb was covered in plastic wrap and the entire surgical and video recording procedure was repeated for the contralateral limb. After completion of the examination of both limbs, the mouse was euthanized by cervical dislocation. Mice were stored at $-20^{\circ} \mathrm{C}$ and disposed of with biohazard waste.

\subsection{Femoral Artery Ligation Surgical Protocol}

Surgeries were performed on C57/B6 mice under aseptic conditions and using Isoflurane anesthesia (see Appendix D for experimental checklist). The mice were kept at a temperature of $35 \pm 1.0^{\circ} \mathrm{C}$ using a rectal-temperature controlled heating pad pad. An initial incision was made on the medial aspect of the hindlimb, directly above the femoral neurovascular bundle. This incision was extended from just beyond the abdominal wall to the beginning of the saphenous artery along the neurovascular bundle. Sterile saline solution was used to irrigate the incision and to prevent muscle desiccation. Connective tissue was blunt dissected. In addition, the connective tissue underneath the fat pad covering the muscular branch was dissected to allow for movement and exposure. Following this dissection the fat pad was pushed aside to identify a site for ligation just distal to the deep femoral artery branch. At this site the nerve was carefully blunt 
dissected away from the femoral artery along a length of $1-2 \mathrm{~mm}$. The vein was meticulously blunt dissected away from the artery to a similar length. The dissected femoral artery was then ligated using sterile 6.0 silk suture. Following ligation, the skin incision was closed using 7.0 polyprepylene suture.

An equivalent incision was made to the contralateral hindlimb, above the femoral neurovascular bundle. Again, sterile saline solution was used to (irrigate) prevent tissue desiccation. Connective tissue was blunt dissected. Contact with the neurovascular bundle was avoided in order to simulate every aspect of the surgery except the vascular insult. Finally, the skin incision was closed up with 7.0 polypropylene suture. The mouse was then given a subcutaneous injection of buprenorphine $(0.075 \mathrm{mg} / \mathrm{kg})$ and allowed to recover on a hot pad until ambulatory. Mice were returned to the vivarium at the end of the day.

\subsection{Femoral Artery Resection Surgical Protocol}

Surgeries were performed on C57/B6 mice under aseptic conditions and using Isoflurane for anesthesia (see Appendix E for experimental checklist). The mice were kept at a temperature of $35 \pm 1.0^{\circ} \mathrm{C}$ using a using a rectal-temperature controlled heating pad. An initial incision was made on the medial aspect of the hindlimb, directly above the femoral neurovascular bundle. This incision was extended from just beyond the abdominal wall to near the ankle of the hindlimb (just below the midway point between the knee and the heel) (Figure 12). Sterile saline solution was used to prevent muscle desiccation. Connective tissue was blunt dissected. The fat pad covering the muscular junction was 
then blunt dissected away from both the muscular tissue below and the abdominal wall. The epigastric artery was then cauterized and a square of the fat pad was then removed to increase exposure of the muscular branch. The nerve within femoral neurovascular bundle was then carefully blunt dissected away from the artery vein pair along the entire length of the femoral artery. After complete separation of the nerve from the artery vein pair, the femoral artery vein pair was dissected from the tissue directly below. The femoral artery-vein pair was then ligated just proximal to the muscular branch with 6.0 silk suture. The muscular branch artery and vein were dissected at the junction from the femoral artery and cauterized. Then the saphenous artery vein pair was ligated at the point half way between the knee and the heel. The vessels were then cauterized below the ligature and the ligature was used to pull up the vascular pair to the first ligature, while cutting and cauterizing vascular branches as necessary. The artery vein pair was cut distal to the first ligature. Finally the skin incision was closed with 7.0 polypropylene suture.

The contralateral limb was then treated the same as the sham surgery for the ligated mice. The mouse was then taken off of anesthesia, given a subcutaneous injection of buprenorphine $(0.075 \mathrm{mg} / \mathrm{kg})$ and allowed to recover on a hot pad until ambulatory. Mice were returned to the vivarium at the end of the day.

\subsection{VASCUlar Function EXPERIMENTS on IschemiC Mice}

Experiments were performed on C57/B6 mice 14 days after each surgery in the same manner as the section, Vascular Function Experiments on Mice Not Surgically Altered 
(see Appendix C for experimental checklist), with a few amendments. For example, in resected mice, the fat pad of the ischemic limb had already been removed. Thus, minor, newly formed adipose only needed to be dissected. Furthermore in these mice, the muscular branch was refractory to vascular tone recovery following the cessation of stimulation. Thus, a frequency of $8 \mathrm{~Hz}$ was always executed first as it was the most important stimulation frequency, because this large frequency should yield a maximal vascular response. Aside from this, stimulation frequency was randomized. Finally, mice underwent ventricular catheterization and perfusion fixation following final video recordings.

\subsection{SDF Imaging ANalysis Protocol Using AVA (Automated VasculaR}

\section{ANALYSIS)}

The MicroScan was first calibrated according to the manufacturer's specifications to ensure accurate data collection. During data collection, the microscope probe was placed such that the planes of the probe and the hindlimb were parallel and that contact between the limb and probe was minimized to reduce any effects forceful physical contact may have on vascular function. To record a video AVA software version 3.0 was opened, calibration settings for the microscan peripheral were loaded and the "capture" option was selected (Figure 17). Mouse information was entered and the video duration was set at 10 seconds. Following either the 30 minutes of rest or 3 minutes of stimulation ten second videos were recorded and saved. 


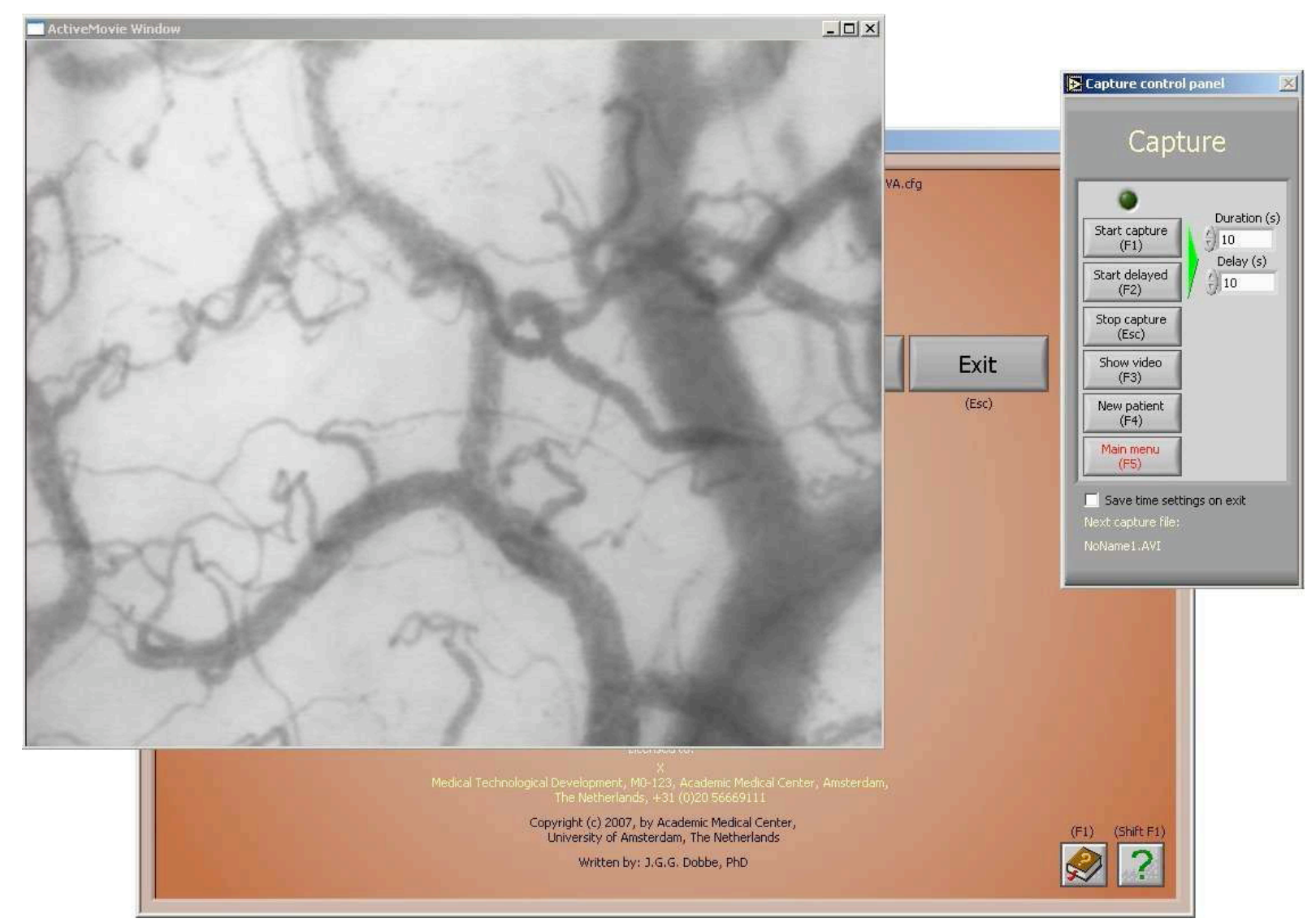

Figure 17. AVA capture workspace. Capture workspace, with capture options and current video recording [63].

After video collection AVA was again opened and the "analysis" option was selected (Figure 18). Once the analysis workbook appeared one of the videos recorded was opened. The video was then stabilized using the automated video stabilization button to reduce any movement that may have occurred during recording due to breathing (mouse) or any other causes. The muscular branch artery is considered a large vessel by the AVA software and large vessels are to be manually traced. Thus the manual tracing tool was selected and the muscular branch in each video was carefully traced segment by segment down the length of the vessel (vessel length varied due to tissue occasionally obscuring a portion of the vessel). Once this segmentation is completed the segments are linked and 
averaged to give an accurate measurement of the vessel diameter. To improve accuracy this process was repeated at 1 ( 30 frames) and 2 seconds ( 60 frames), and the three diameters were averaged.

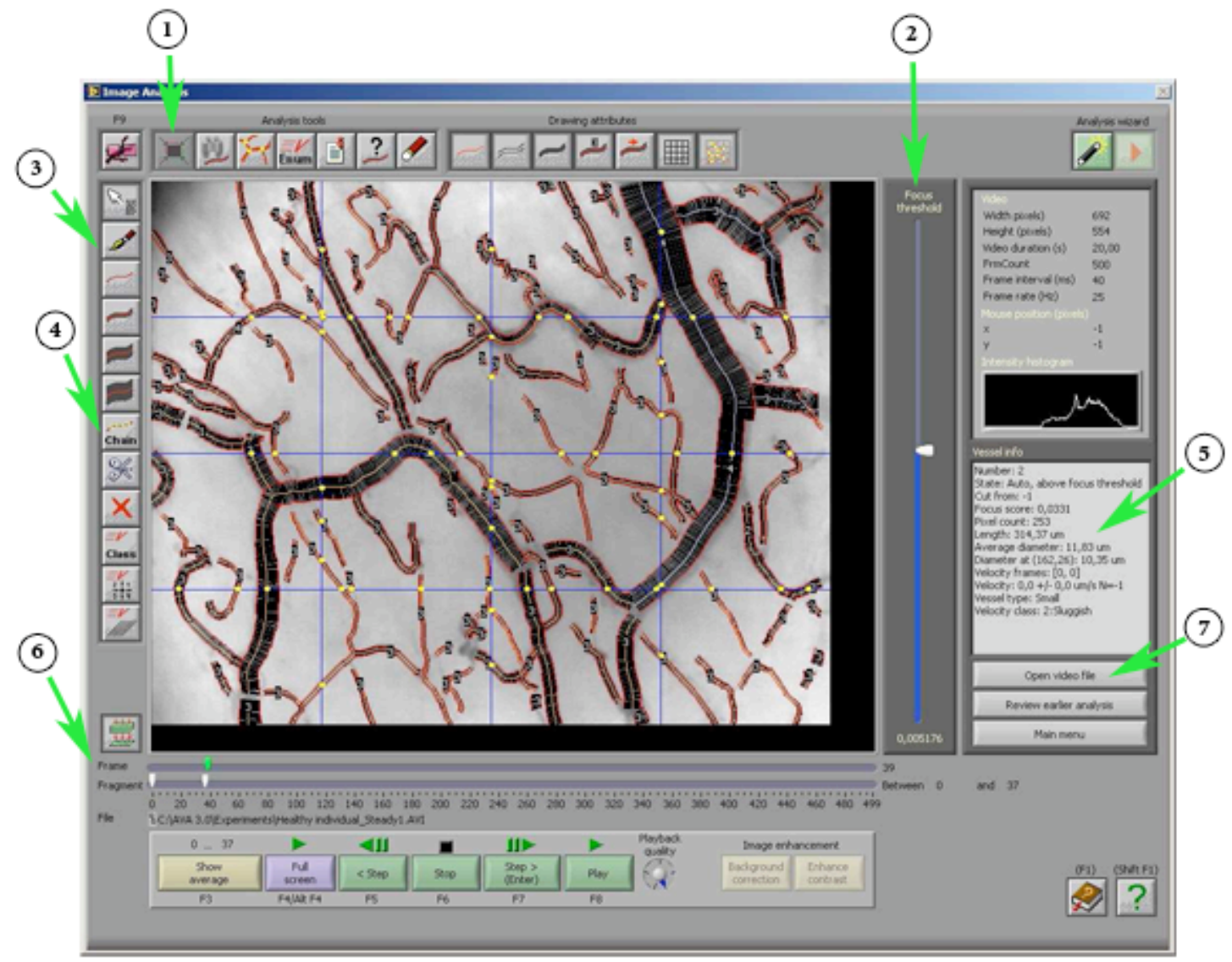

Figure 18. AVA analysis workspace. The workspace has controls for video stabilization (1), focus adjustment (2), manual tracing (3), connecting vessel segments (4), moving between video frames (6), opening video files (7). Vessel output is then displayed when each vessel segment is selected (5) (adapted from [63]). 


\subsection{STATISTICAL ANALYSIS}

Vasodilation in healthy animals was evaluated by examining muscular branch artery diameter changes after gracilis stimulation at various frequencies. Statistical significance between each condition in the same mouse was assessed using the Tukey-Kramer test. Factors such as mouse ID (i.e. the number that distinguishes each mouse), limb order and right/left limb were assessed using balanced ANOVA and a paired means t-test. Vascular function and repair after each surgery was evaluated by looking at both arterial diameter (analyze any remodeling) and vasodilation (see Equation 1). Percent diameter increase was used to demonstrate how much the artery could expand relative to its resting

$$
\text { Equation 1. } \quad \text { Vasodilation }_{\%_{\text {olncrease }}}=\left(\frac{\text { Diameter }_{\text {stimulated }}}{\text { Diameter }_{\text {rest }}}-1\right) \times 100
$$

diameter. Vasodilatory ability and remodeling comparisons between the sham, ligation and resection surgeries were analyzed using balanced ANOVA, a paired means t-test (limbs on the same mouse and exposed to the same stimulation frequency) and differences between each condition (surgery and frequency) were assessed using a t-test based on the differences between contralateral limbs. 


\section{RESULTS}

\subsection{FunCtional Hyperemia in Healthy Animals}

In accordance objective 1 (pg. 29), an SDF imaging protocol was established to evaluate functional vasodilation in the muscular branch artery. Stimulation of the obturator nerve and contraction of the gracilis muscles resulted in maximal vasodilation at a frequency of $8 \mathrm{~Hz}$ (Figure 19). Specifically, gracilis stimulation induced a significant increase in arterial diameter from $64.8 \pm 1.9 \mu \mathrm{m}$ to $118.4 \pm 4.7 \mu \mathrm{m}$ at $8 \mathrm{~Hz}, \mathrm{p}<0.001(\mathrm{n}=10)$. Furthermore, each stimulation frequency elicited significantly different levels of vasodilation, except between $4 \mathrm{~Hz}$ and $8 \mathrm{~Hz}$, where there was an increase in dilation, but not a statistically significant difference.

Further, this maximal vasodilation was statistically significant relative to the resting diameter as there was nearly a $100 \%$ increase in arterial diameter. The data demonstrates a relationship between frequency of muscle stimulation and vascular diameter.

Interestingly, the order in which the hindlimbs were evaluated was statistically significant to the corresponding levels of vasodilation (percentage increase) (Figure 20). The hindlimb examined first demonstrated enhanced vasodilation compared to that imaged later $(78 \%$ compared to $56 \%$ increase, $\mathrm{p}=0.006)$. This was important for future experiments as limb order continued to be randomized. 
A

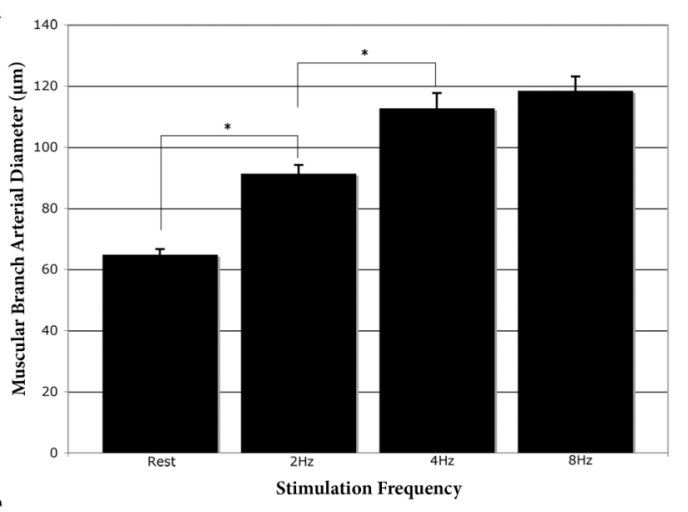

C

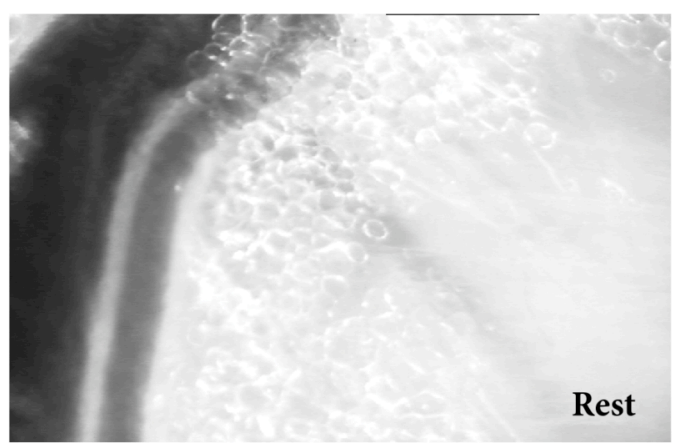

B

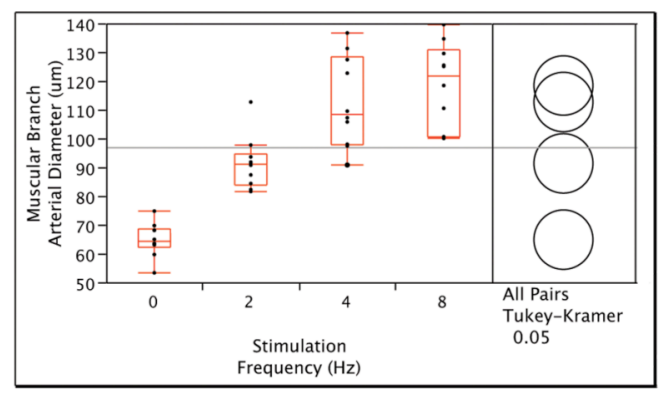

D

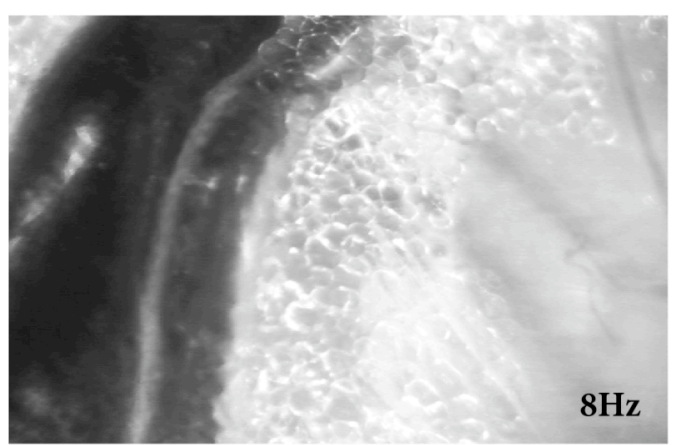

Figure 19. Vasodilation in healthy mice. A bar graph of the mean muscular branch artery luminal diameter following gracilis stimulation at $1 \mathrm{~mA}, 500 \mu \mathrm{s}$ and $2 \mathrm{~Hz}, 4 \mathrm{~Hz}$ or $8 \mathrm{~Hz}$ for $3 \mathrm{~min}(\mathrm{~A})\left({ }^{*} \mathrm{p}<0.001\right)$. A chart showing the quantiles and distribution of arterial diameters at each condition and a Tukey-Kramer diagram showing significant differences $(\mathrm{p}<0.05)$ where the circles do not overlap (B). Single frames taken by the MicroScan of the muscular branch artery during rest (C) and following stimulation of the gracilis muscle at a frequency of $8 \mathrm{~Hz}(\mathrm{D})$. 
A

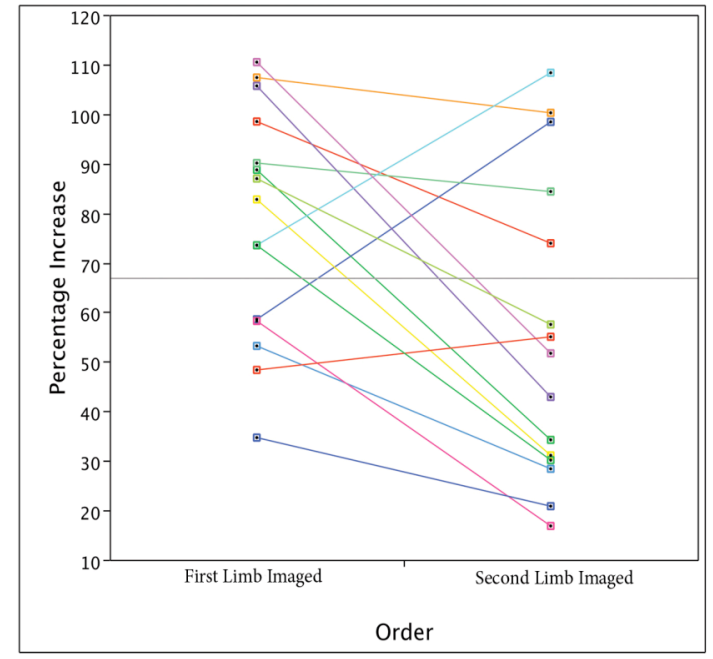

B

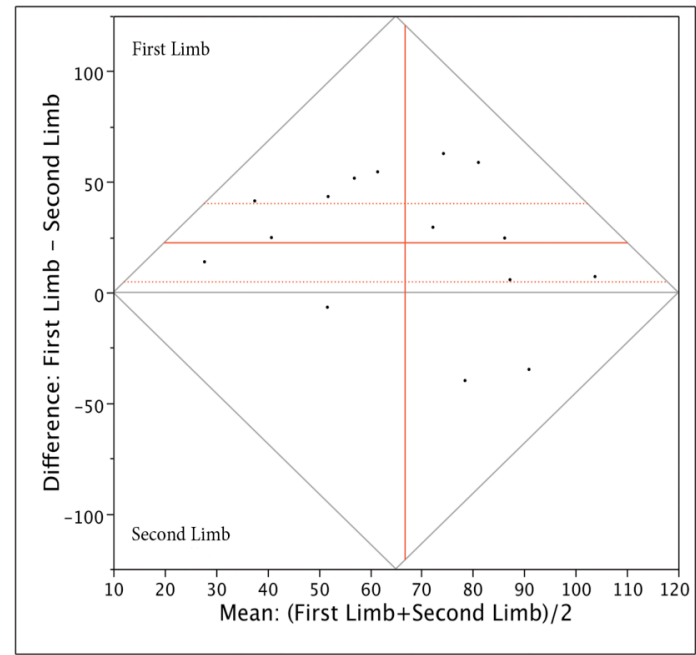

Figure 20. The importance of limb order. A distribution of vasodilation levels in the first and the second hindlimb imaged, which connects data points between limbs on the same mouse and vasodilation levels at the same frequency (A), showing that in most experiments vasodilation levels decreased between the first and second limb imaged. A depiction of a paired t-test done for vasodilation levels in the first vs. second limb imaged at a certain frequency (B). The horizontal dotted red lines show the $95 \%$ interval for the difference between the first and second limb, demonstrating that the higher levels of vasodilation in the first limb imaged was statistically significant $(\mathrm{p}<0.05)$.

\subsection{OUtWARd REMODELING AND VASODILATION FolLOWING ARTERIOGENIC ISCHEMIA}

As expected, the muscular branch artery luminal diameter was larger in the ligated hindlimb or the hindlimb that underwent femoral artery ligation. The resting diameters of ligated hindlimbs were $72.0 \pm 3.6 \mu \mathrm{m}$ compared to $61.3 \pm 2.1 \mu \mathrm{m}$ in the non-ischemic contralateral limbs (Figure 21). In addition, the arterial diameters following gracilis stimulation at $8 \mathrm{~Hz}$ were $120.0 \pm 4.8 \mu \mathrm{m}$ and $105.9 \pm 1.3 \mu \mathrm{m}$ in ligated and non-ischemic limbs respectively. 
A
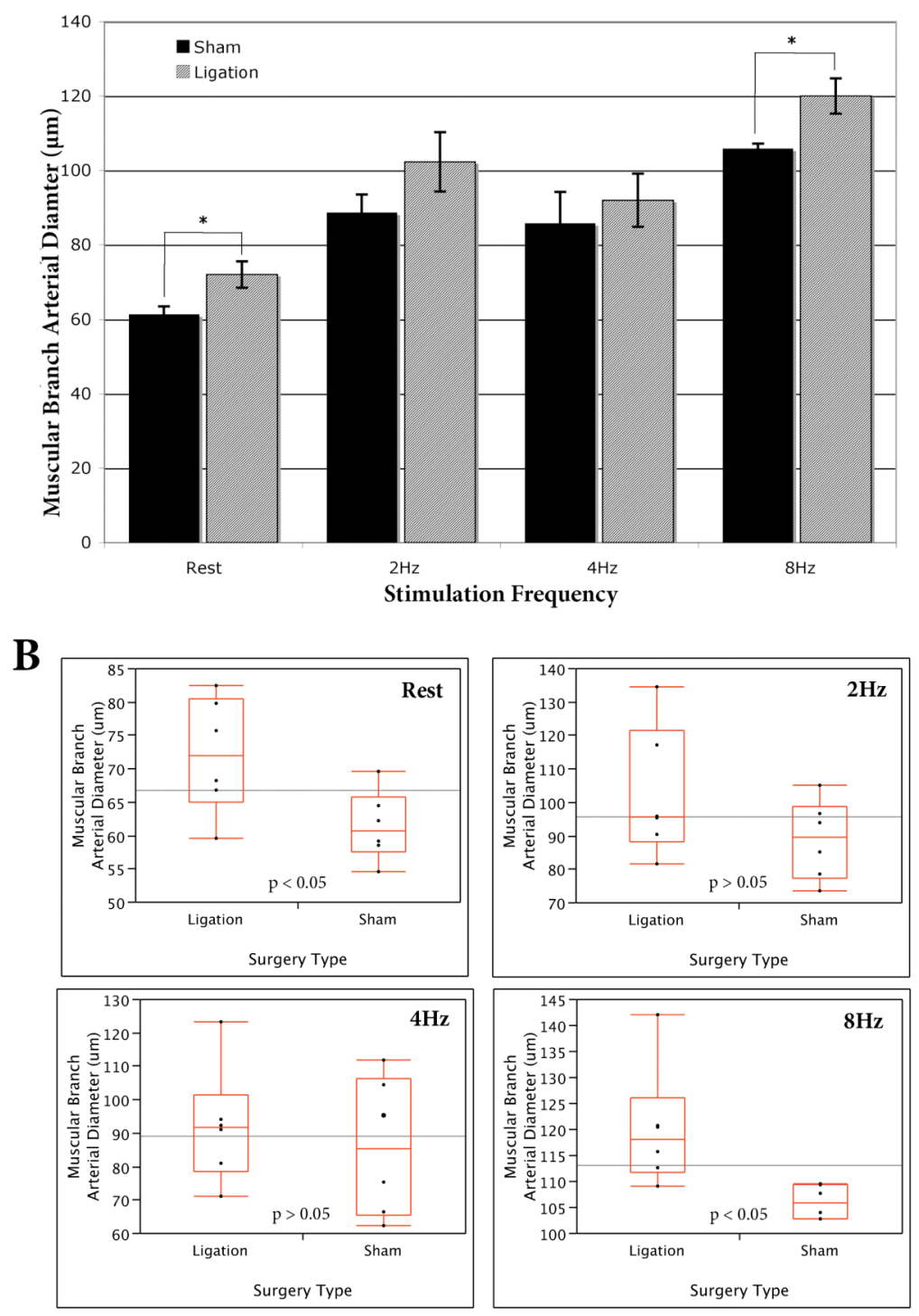

Figure 21. Arteriogenesis of the muscular branch artery in ligated mice. A bar graph comparing the muscular branch artery diameter in the ligated ischemic and non-ischemic hindlimb at rest and following stimulation at $1 \mathrm{~mA}, 500 \mu \mathrm{s}$ and $8 \mathrm{~Hz}$ for $3 \mathrm{~min}(\mathrm{n}=6)(\mathrm{A})$. These observations were recorded on day 14 following ligation and sham surgeries on contralateral limbs of C57/B6 mice. Charts showing the distribution of arterial diameter values following sham and ligation surgeries at various frequencies (B) demonstrate that the muscular arterial diameter following the ligation surgery is significantly larger than that of the sham surgery at rest and at maximal dilation (also see Figure 23 for examples of the videos this data was based on). 
Vascular function was assessed, in the muscular branch of the ligated mice and compared to the same vasculature in the non-ischemic hindlimb. There was no significant difference in vascular function of the remodeled muscular branch artery when compared to the non-repaired muscular branch (Figure 22).

Figure 22. Vasodilation of the muscular branch artery in ligated mice. A bar graph showing muscular branch artery diameter increase $(\%$ increase $)$ at $1 \mathrm{~mA}$, $500 \mu \mathrm{s}$ and $2 \mathrm{~Hz}, 4 \mathrm{~Hz}$ or $8 \mathrm{~Hz}$ for $3 \mathrm{~min}$, in limbs sustaining either a sham or femoral artery ligation surgery (A). Also shown, a chart of the distribution of arterial vasodilation in paired limbs (i.e. same mouse, same stimulation, one ligated and one sham), which shows no significant change in vasodilation between limbs receiving a sham surgery and those receiving a ligation
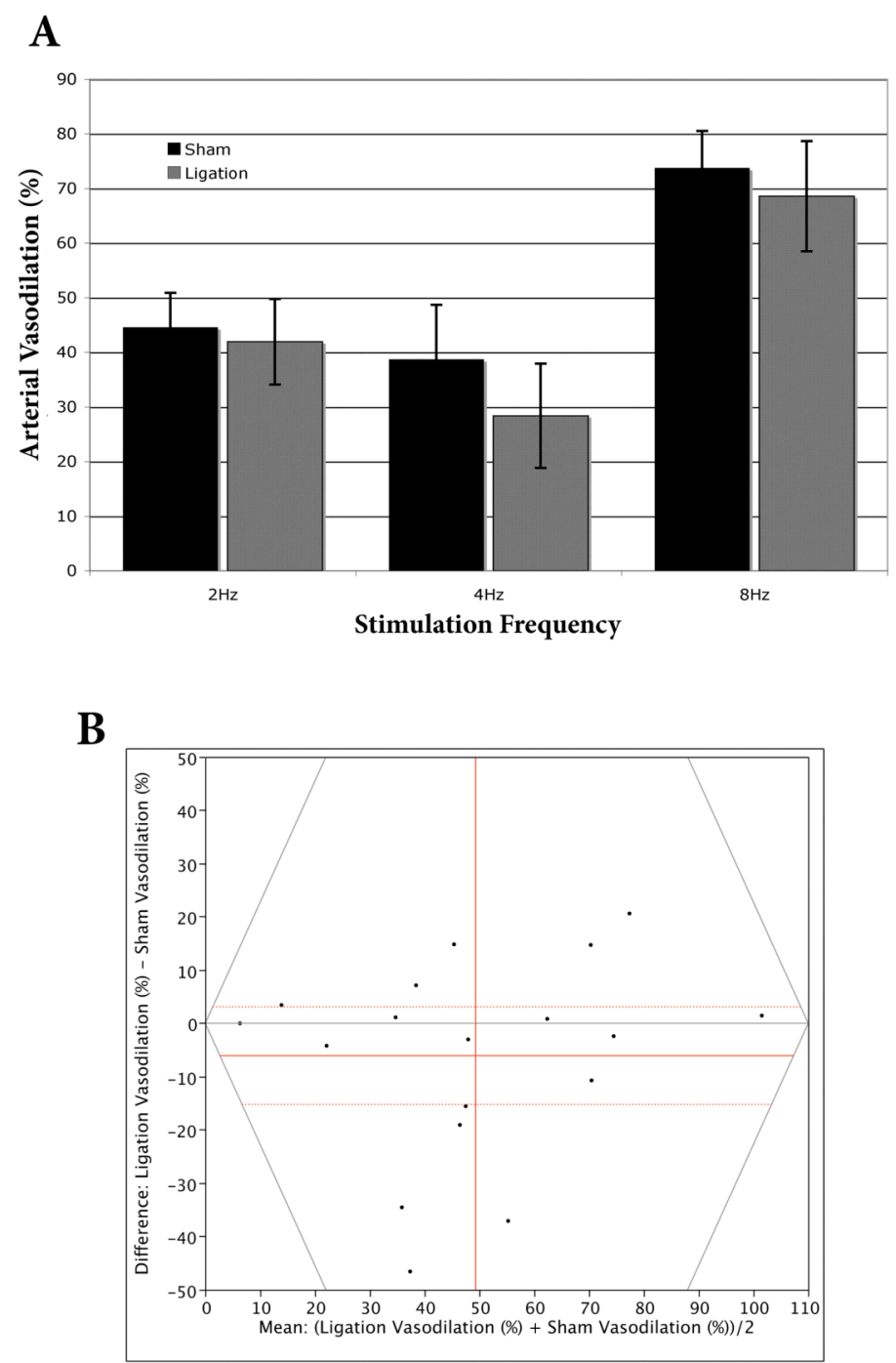
surgery (dotted red lines indicate $95 \% \mathrm{CI}$ ). This data was evaluated on day 14 following surgery on both the ligated and non-ischemic hindlimbs (also see Figure 23 for examples of the videos this data was based on). 
A

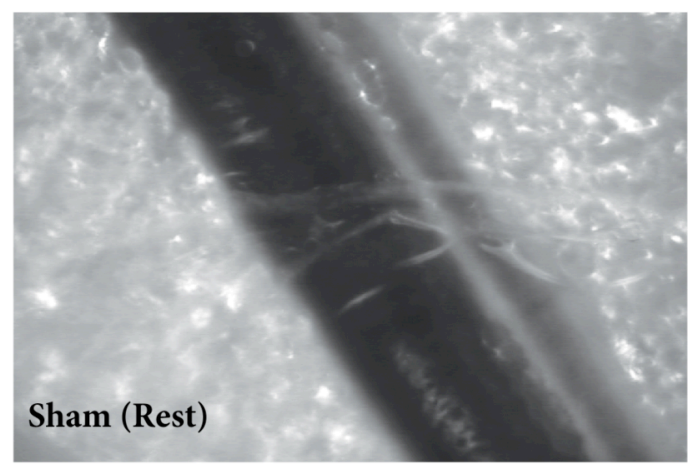

C

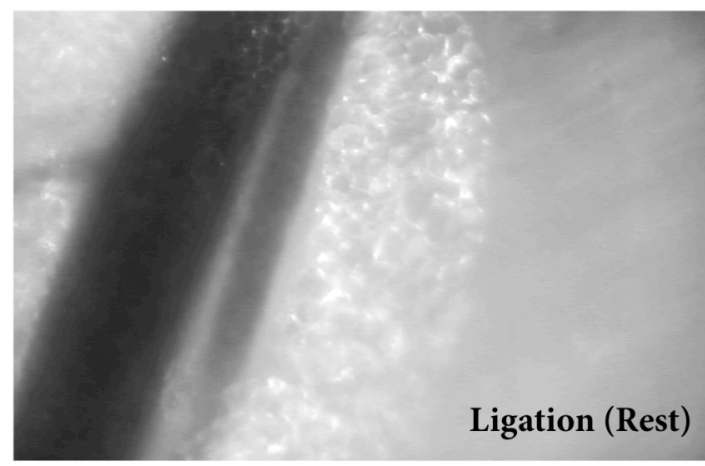

B

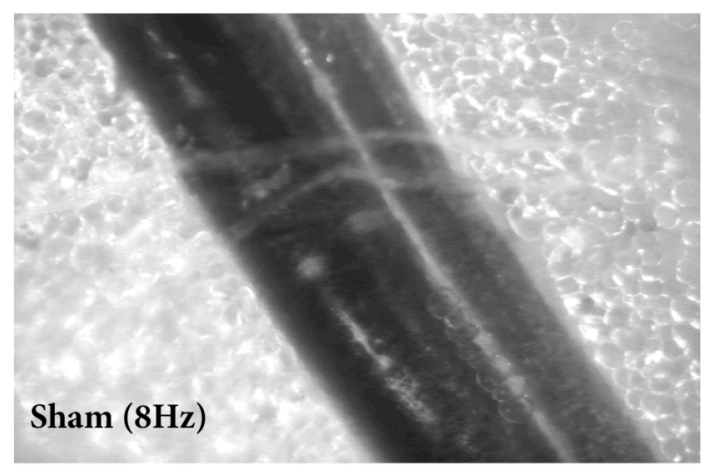

D

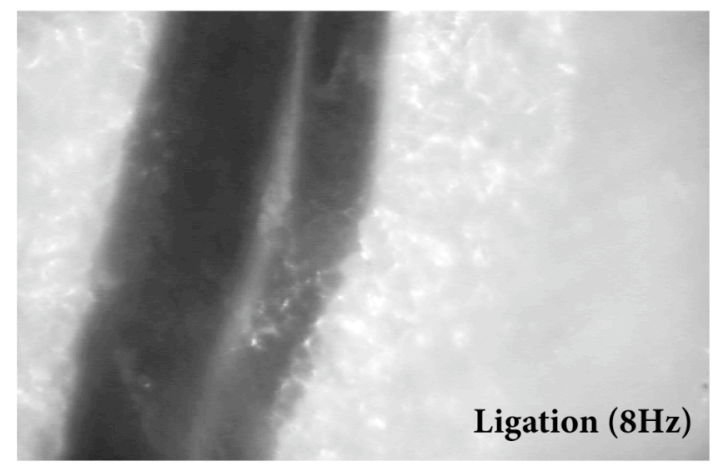

Figure 23. Images of muscular branch artery changes in ligated mice. Samples freeze frames from videos recorded of the muscular branch artery of a non-ischemic hindlimb at rest (A) and following a gracilis stimulation of $8 \mathrm{~Hz}(\mathrm{~B})$, in addition to freeze frames from videos recorded of the muscular branch artery of the ligated contralateral hindlimb at rest (C) and following a gracilis stimulation of $8 \mathrm{~Hz}(\mathrm{D})$.

\subsection{Arterial Resting Tone and Vasodilation Following Non-Arteriogenic}

\section{ISCHEMIA}

In contrast, in those mice that experienced femoral artery resection, the muscular branch arterial diameter was not statistically different from the diameter of the equivalent artery on the contralateral non-ischemic limb (Figure 24). 
A

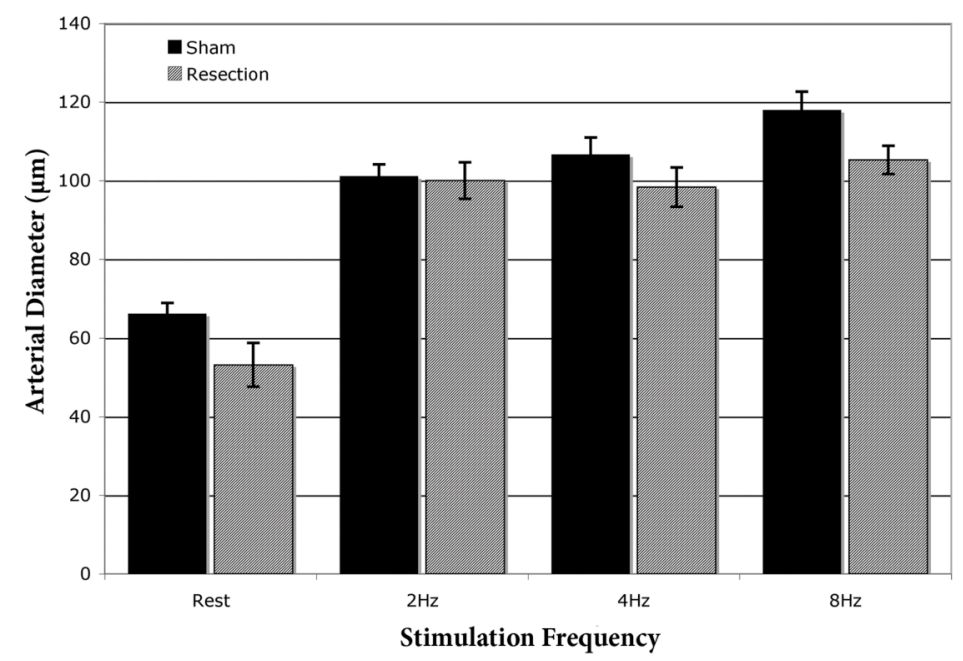

B

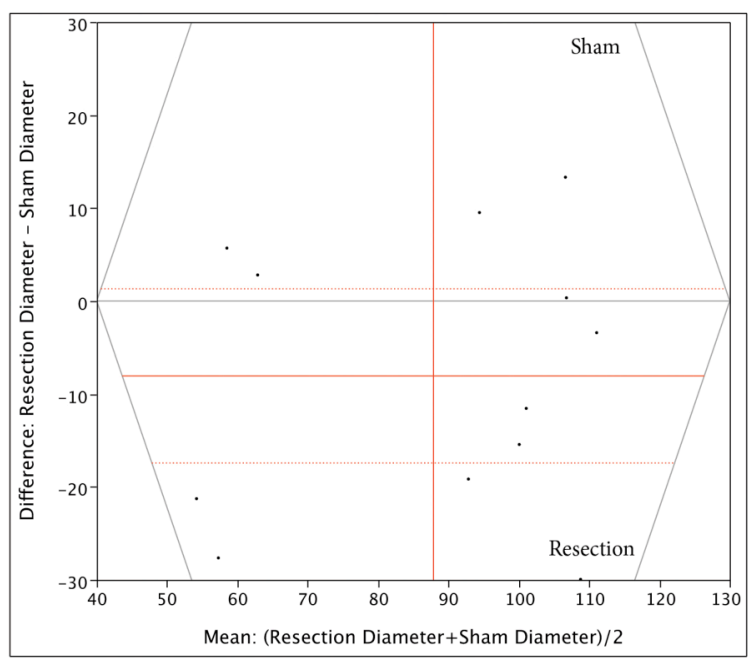

Figure 24. Neutral remodeling of the muscular branch artery in resected mice. Muscular branch artery luminal diameters in the resected ischemic and non-ischemic hindlimb at rest and following stimulation at $1 \mathrm{~mA}, 500 \mu \mathrm{s}$ and $2 \mathrm{~Hz}, 4 \mathrm{~Hz}$ or $8 \mathrm{~Hz}$ for $3 \mathrm{~min}(\mathrm{n}=4)(\mathrm{A})$. At each condition there is not a significant difference in diameter. Also shown, a chart of the distribution of arterial diameters in paired limbs (i.e. same mouse, same stimulation, one resected and one sham), which shows no significant change in arterial diameters between limbs receiving a sham surgery and those receiving a resection surgery (dotted red lines indicate $95 \% \mathrm{CI}$ ). This data was evaluated on day 14 following surgery on both the resected and nonischemic hindlimbs (also see Figure 26 for examples of the videos this data was based on). 
Vascular function was also analyzed in the muscular branch artery of resected mice and compared to that in the non-ischemic contralateral limb. There appeared to be no significant trend indicating a change in vascular function in the repaired resected hindlimb compared to the non-ischemic hindlimb (Figure 25). However, during the experiments it was clear that the muscular branch artery in the resected hindlimb was refractory to regaining resting vascular tone following the cessation of stimulation. Normally, a wait of 25-30 minutes, if not longer, was needed for the muscular branch arterial diameter to return close to its resting diameter. This is a significant increase in time when compared to the 4-8 minutes that were required for the muscular branch artery to constrict in both the ligated and non-ischemic hindlimbs. Unfortunately, this observation was only observed qualitatively during experiments and thus there are not any data on a potential impairment of vascular function, specifically concerning the recovery of resting vascular tone. This may be an observation that deserves further examination. 


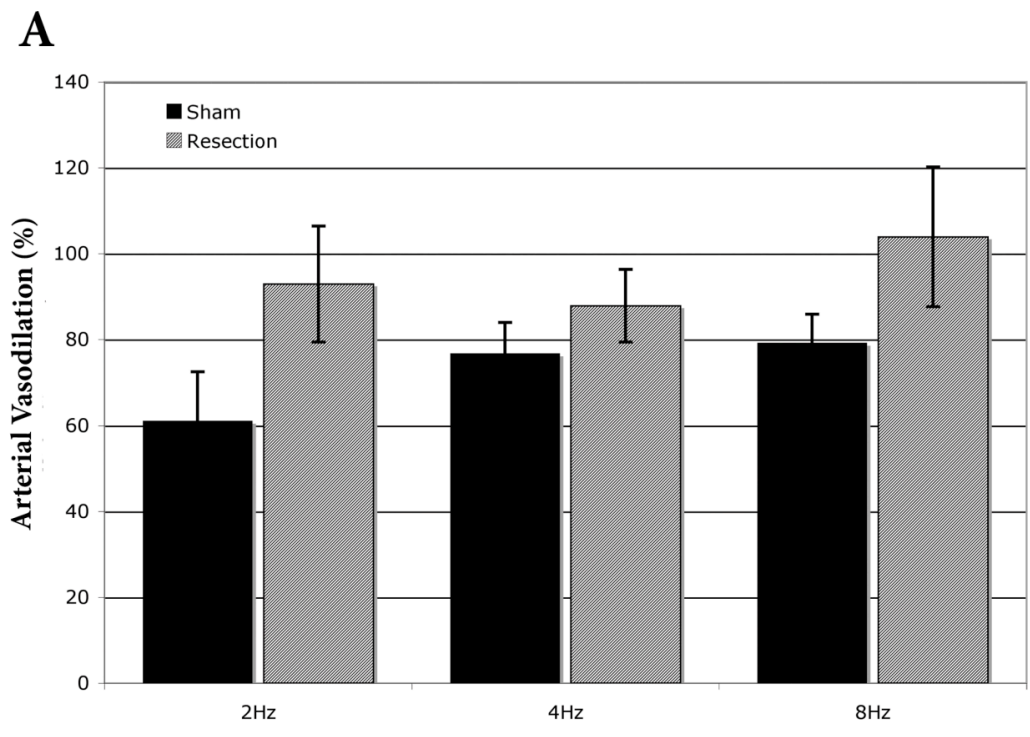

Stimulation Frequency

B

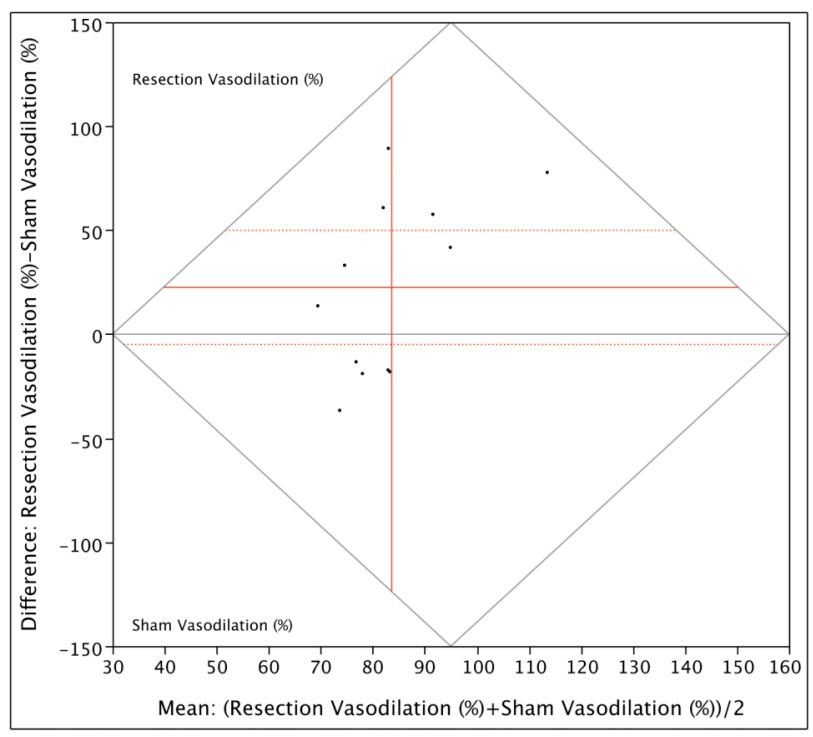

Figure 25. Vasodilation of the muscular branch artery in resected mice. Muscular branch artery diameter increase (percentage increase relative to resting diameter) at $1 \mathrm{~mA}, 500 \mu \mathrm{s}$ and $2 \mathrm{~Hz}, 4 \mathrm{~Hz}$ or $8 \mathrm{~Hz}$ for $3 \mathrm{~min}$, in limbs sustaining either a sham or femoral artery resection surgery (A). Also shown, a chart of the distribution of arterial vasodilation in paired limbs (i.e. same mouse, same stimulation, one resected and one sham), which shows no significant change in vasodilation between limbs receiving a sham surgery and those receiving a resection surgery (dotted red lines indicate 95\% CI). This data was evaluated on day 14 following surgery on both the resected and non-ischemic hindlimbs (also see Figure 26 for examples of the videos this data was based on). 
A

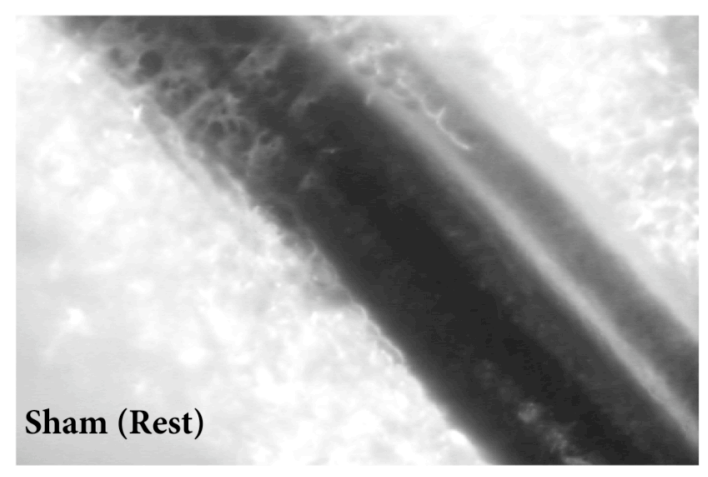

C

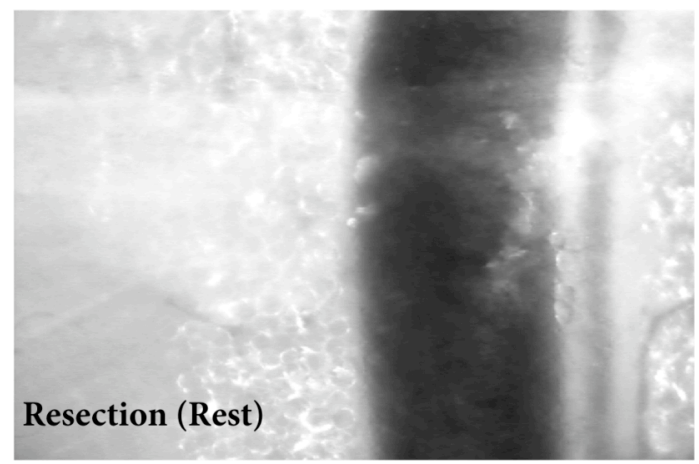

B

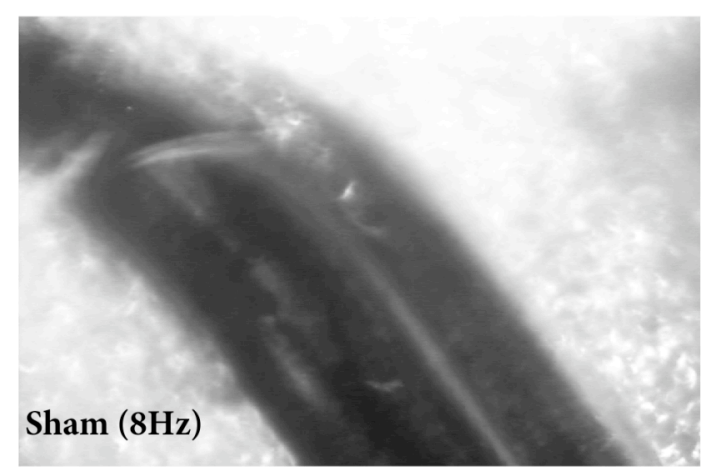

D

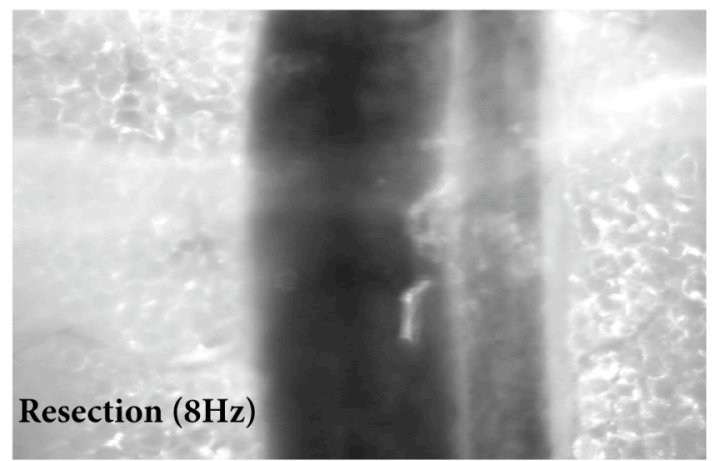

Figure 26. Images of muscular branch artery changes in resected mice. Samples freeze frames from videos recorded of the muscular branch artery of a non-ischemic hindlimb at rest (A) and following a gracilis stimulation of $8 \mathrm{~Hz}(\mathrm{~B})$, in addition to freeze frames from videos recorded of the muscular branch artery of the resected contralateral hindlimb at rest (C) and following a gracilis stimulation of $8 \mathrm{~Hz}(\mathrm{D})$. 


\section{DISCUSSION}

\subsection{RESTATEMENT OF EXPERIMENTAL OBJECTIVES}

To guide the discussion of experimental results, recall the primary goals:

1. Establish a consistent protocol for the SDF imaging intravital microscope to examine muscular branch arterial changes during functional hyperemia induced by gracilis stimulation.

2. Examine (using the established SDF imaging protocol) collateralization of the muscular branch artery two weeks after ischemic surgeries.

3. Examine vascular functional hyperemia following arteriogenic and nonarteriogenic ischemic insult.

\subsection{Vasodilatory Response in Healthy Mice}

An SDF imaging protocol was established to consistently evaluate the hindlimb vasculature (and will be discussed). Functional vasodilation in the muscular branch artery as a result of gracilis muscle contraction was examined following stimulation of the obturator nerve. As expected, isometric gracilis muscle contraction induced an increase in the diameter of the muscular branch artery, so to augment blood flow and satiate metabolic demands. Furthermore, there was a stepwise enhancement of the vasodilatory response when the stimulation frequency was increased from $2 \mathrm{~Hz}$ to $4 \mathrm{~Hz}$ to $8 \mathrm{~Hz}$. A similar response was observed in previous examinations of the rat spinotrapezius muscle 
(as well as other muscles), where an increasing electrical stimulation frequency elicited progressively elevated distension of vasculature diameter in the aforementioned muscle [64]. This reaction observed both in the gracilis and spinotrapezius muscles of the mouse and rat, respectively, is practically relevant to muscle and circulatory function during exercise; in these experiments increasing the frequency of muscle contractions was analogous to escalating the intensity of exercise in vivo. In this way, amplification of muscle activity results in elevated metabolic demand as muscles both consume and require more nutrients. To quench this demand, perfusing arteries dilate in order to increase nutrients and oxygen supplied to contracting skeletal muscle via blood flow augmentation. The evaluation of vasodilatory function in the muscular branch artery was necessary to ensure that the established dissection, stimulation and imaging protocol was capable of acquiring consistent results and observing the expected arterial response like that observed in the rat spinotrapezius [64].

\subsection{SDF Imaging Protocol Modifications}

Following some protocol revisions, the SDF imaging microscope was capable of consistently observing and recording arterial diameter changes in the muscular branch artery. Initially, vasodilation data acquired yielded high variability and error, thus a number of key modifications were needed to address these problems (Figure 27). The most important procedural consideration was to increase the allowed recovery time following tissue dissection and prior to muscle stimulation and SDF video imaging from 15 to 30 minutes. This time was imperative to allow for the recovery of vascular tone following dissection and exposure. If this time was not allotted a number of problems 
occurred. The resting diameter of the muscular branch artery was not always reached, as the artery did not fully regain resting vascular tone. In addition, there were instances when the artery was refractory to the stimulus of muscle contraction and would not vasodilate to meet the metabolic demands of the gracilis muscles. Specifically, referring to instances involving the muscular branch of non-ischemic and resected hindlimbs, the muscular branch artery only dilated from $71.94 \mu \mathrm{m}$ (resting) to $90.11 \mu \mathrm{m}(8 \mathrm{~Hz})$ and $76.160 \mu \mathrm{m}$ (resting) to $87.09 \mu \mathrm{m}(8 \mathrm{~Hz})$, respectively. This level of dilation was especially low when compared to other observed results. Furthermore, these instances did not appear to occur during conditions when the force of gracilis contraction were was seemingly depressed. These instances of abnormal vasodilation combined with the more common inability for the muscular branch to regain resting diameter following dissection led to inconsistent and inconclusive results.

While extending the recovery time prior to nerve stimulation from 15 minutes to 30 minutes ameliorated these negative results, the protocol also needed to be adapted to address the difficulties involved with placing this large (relative to hindlimb) SDF probe such that the horizontal imaging plane was parallel to the hindlimb surface in order to reduce contact with the muscular branch artery. Given the aforementioned large size of the probe and the modest flexibility of the abdominal wall, it was slightly problematic to position the microscope in the correct location for muscular branch artery visualization without the probe placing pressure on the hindlimb. To remedy this problem, special attention was placed to probe positioning and the hindlimb was leveled in the horizontal plane as much as possible using a combination of cotton swabs, tongue depressors and 
metal spatulas. This was approached with two objectives in mind: to ensure that the probe would not contact the muscular branch artery or other hindlimb microcirculation and that the probe would not interfere

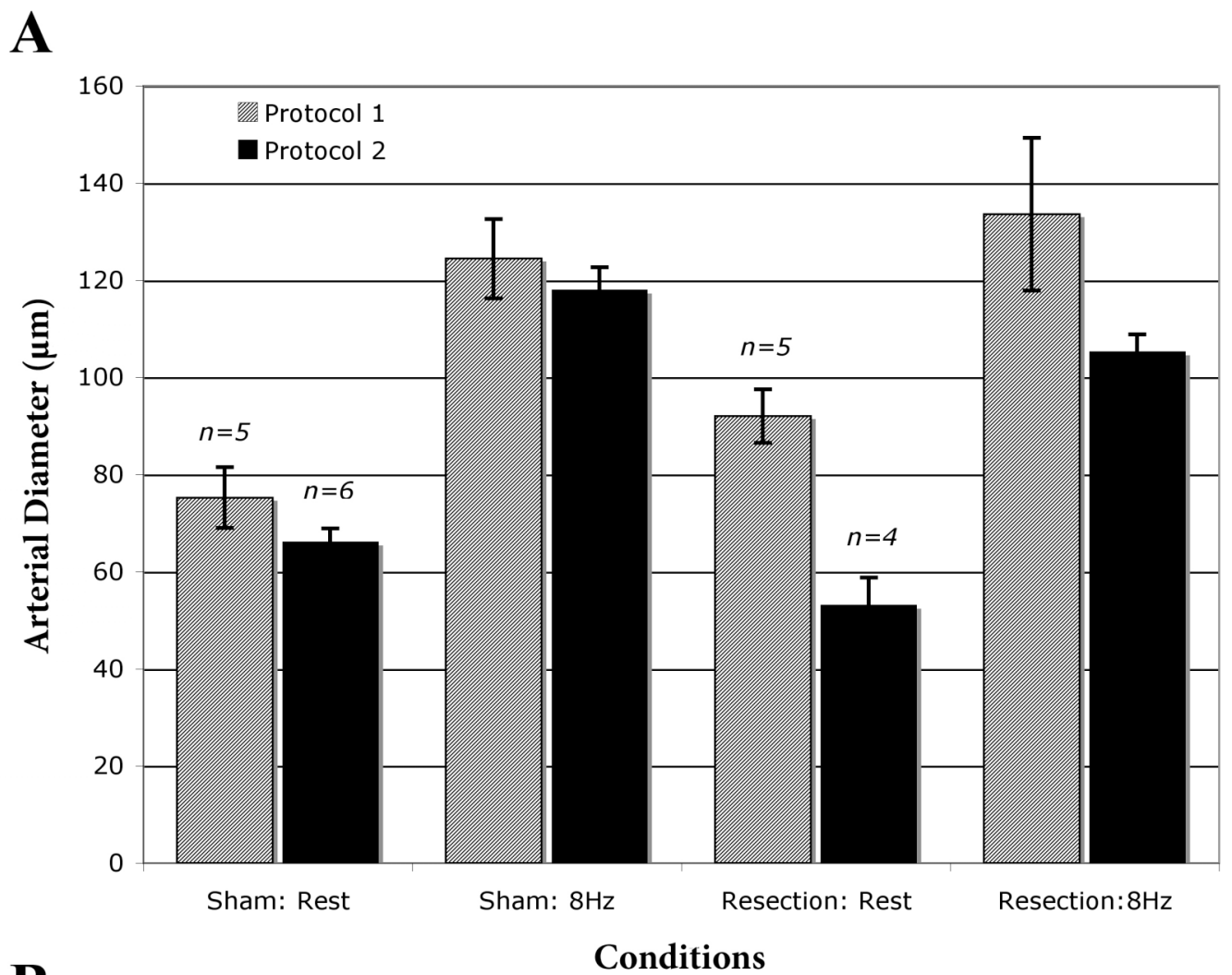

B

\begin{tabular}{|l|c|c|c|c|c|c|c|c|}
\hline $\begin{array}{l}\text { Surgery } \\
\text { Type }\end{array}$ & \multicolumn{4}{|c|}{ Ligation } & \multicolumn{5}{c|}{ Resection } \\
\hline Hindlimb & \multicolumn{2}{|c|}{ Sham } & \multicolumn{2}{c|}{ Ischemic } & \multicolumn{3}{c|}{ Sham } & \multicolumn{2}{c|}{ Ischemic } \\
\hline $\begin{array}{l}\text { Stimulation } \\
\text { Frequency }\end{array}$ & $0 \mathrm{~Hz}$ & $8 \mathrm{~Hz}$ & $0 \mathrm{~Hz}$ & $8 \mathrm{~Hz}$ & $0 \mathrm{~Hz}$ & $8 \mathrm{~Hz}$ & $0 \mathrm{~Hz}$ & $8 \mathrm{~Hz}$ \\
\hline $\begin{array}{l}\text { Arterial } \\
\text { Diameter } \\
(\mu \mathrm{m})\end{array}$ & 71.94 & 90.11 & 77.80 & 114.03 & 84.77 & 153.33 & 100.42 & 106.32 \\
\cline { 2 - 10 } & 67.89 & 107.38 & 88.89 & 115.70 & 78.36 & 104.96 & 91.37 & 162.57 \\
\cline { 2 - 10 } & 74.89 & 115.34 & 69.70 & 153.27 & 64.98 & 116.68 & 76.16 & 87.09 \\
\cline { 2 - 10 } & 71.72 & 105.58 & 66.37 & 94.07 & 90.98 & 129.12 & 107.17 & 146.20 \\
\cline { 2 - 9 } & 65.30 & 125.13 & 83.60 & 114.07 & 57.31 & 117.96 & 84.77 & 165.77 \\
\hline
\end{tabular}

Figure 27. Inconsistent data from original imaging protocol. (A) Muscular branch artery vasodilation measurements prior to protocol modifications (Protocol 1) and after protocol modifications (Protocol 2). The standard error during most conditions was reduced by at least half of the previous error when the new protocol was utilized. (B) A table of a collection of data collected when using the initial protocol. 
with gracilis muscle contractions. While this was effective, methods were considerably improvisational. Thus, creating a more uniform method to address these concerns would be in the interest of future experiments with the SDF intravital microscope. Specifically a microscope stand made for the SDF microscope would be especially useful as a replacement for the chemistry clamp stand that only had fine adjustment capability in the z-direction. A stand would better secure the microscope and allow for improved fine adjustments when trying to navigate the hindlimb without interfering with the tissues and vasculature.

\subsubsection{Potential Protocol Adaptations yet to be Addressed}

There were other inconsistent aspects of the protocol that were identified and considered but have yet to be addressed. First, while the induction of gracilis muscle contractions through stimulation of the obturator nerve improved contraction strength consistency compared to simply piercing the muscle groups and stimulating them directly, muscle contraction strength variation was still observed. It was clear during the experiment that the same stimuli would at times induce stronger muscle contractions where the entire hindlimb would move and at other times induce weaker gracilis muscle contractions generally only visible through the microscope. For example in one healthy control mouse the arterial diameter was $112.6 \mu \mathrm{m}$ in the left hindlimb and $90.7 \mu \mathrm{m}$ in the right hindlimb following the same stimulus of $2 \mathrm{~Hz}$. In another healthy control mouse the muscular branch arterial diameter was $122.7 \mu \mathrm{m}$ in the left limb and $90.7 \mu \mathrm{m}$ in the right limb at a frequency of $4 \mathrm{~Hz}$. Often, it appeared as though the limb containing the muscular branch artery that dilated more experienced more forceful gracilis contractions. 
This data was likely not caused by which limb was being imaged, because the limb being imaged (left or right) did not have a significant influence on vasodilation ( $\mathrm{P}=0.432)$ (this is distinct from the order limbs were imaged: first or second). When the stimulation frequency was at $8 \mathrm{~Hz}$ this variation in vasodilation was observed with a lesser degree of incidence. This is thought to be, because at a frequency of $8 \mathrm{~Hz}$ it was likely that the stimulus was inducing the maximal dilatory response from the vessel. Previous research demonstrated that arterioles in the hamster cremaster muscle exhibited maximal dilation in response to electrical stimulus at $8 \mathrm{~Hz}$ [65]. Based on Figure 28 taken from the aforementioned hamster study, slight inconsistencies in the applied stimuli during low frequency and long-term stimulation would result in more significant diameter changes compared with variations induced by inconsistencies during higher frequency stimulation. In terms of the protocol, changes in the effective stimulation elicited by the electrode may have been due to variation in electrode placement, specifically, contact with the obturator nerve may have influenced the ability for the same frequency and amplitude to excite the nerve to the same degree. Additionally, it is unclear to what degree the obturator nerve was damaged upon dissection and any variation in the amount of tissue damage may have also led to inconsistencies. Again, based on Figure 28, these inconsistencies would likely be less obvious at $8 \mathrm{~Hz}$ when a maximal vasodilatory response is elicited. 


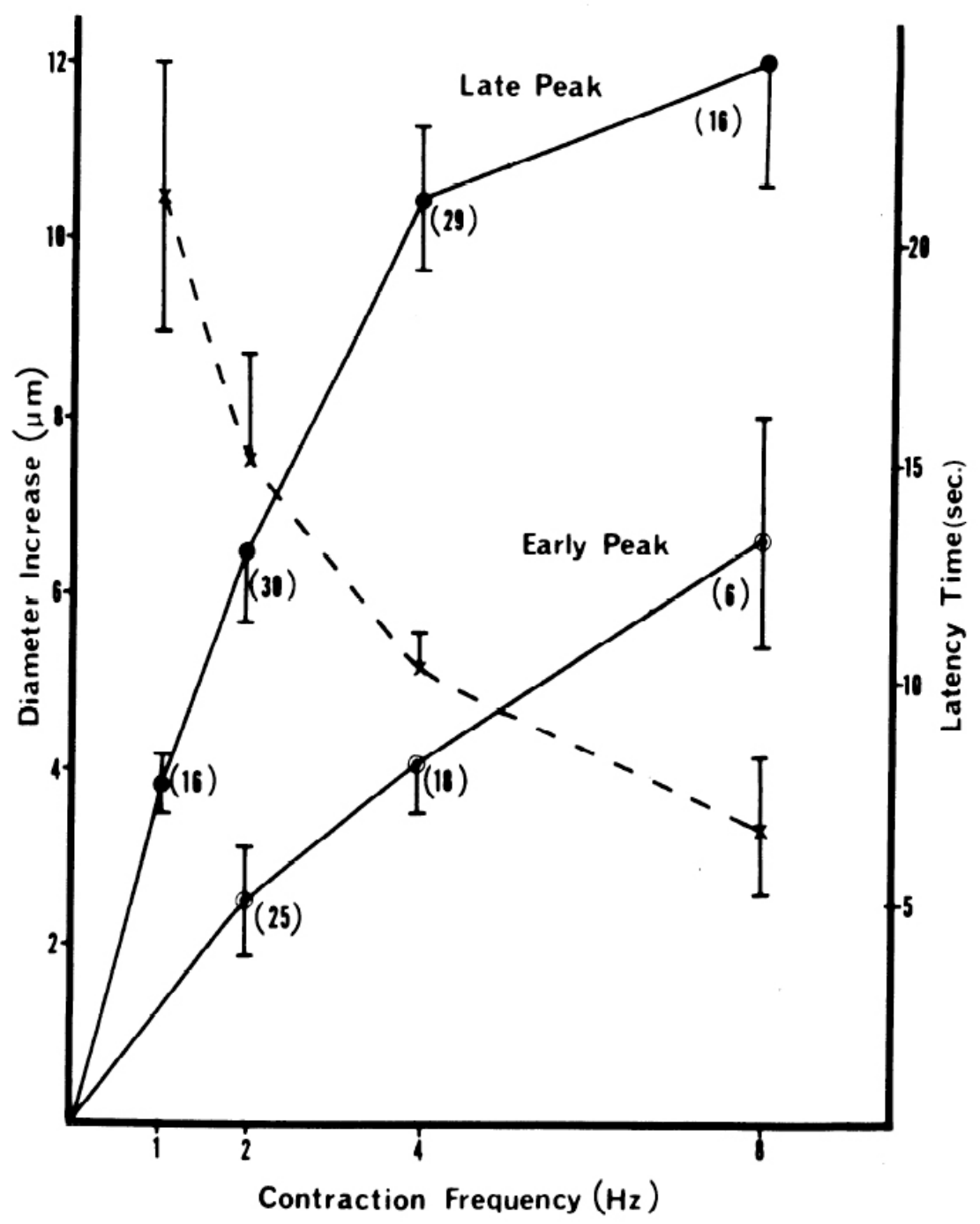

Figure 28. Arteriole vasodilation in the cremaster. The late peak occurred on average after 100 seconds of stimulation and thus is most analogous to the mouse hindlimb observations where the gracilis was stimulated for 180 seconds [65].

Muscle fatigue also appeared to affect the magnitude of these contractions. Even when the contractions were large at the beginning of stimulation and analysis as the experiment progressed there appeared to be a marked decrease in the strength of the muscle contractions. This fatigue was also apparent when evaluating vascular function. At times a weaker stimulation frequency induced a larger change in arterial diameter than a higher 
stimulation frequency if the weaker frequency was executed first. For example, in one normal mouse the muscular branch arterial diameter was $109.5 \mu \mathrm{m}$ following a stimulation frequency of $4 \mathrm{~Hz}$ compared to $100.0 \mu \mathrm{m}$ following a stimulation frequency of $8 \mathrm{~Hz}$ when the $4 \mathrm{~Hz}$ stimulation was completed first.

\subsubsection{Future Protocol Improving Modifications}

To remedy these inconsistencies in muscle stimulation and resultant arterial vasodilation a number of these matters can be addressed. A parallel bipolar electrode with a curved tip to hook the obturator nerve, instead of a straight microelectrode would help to make nerve stimulation and the resultant muscle contraction more consistent. Specifically the hooked aspect of the electrode would limit any movement caused by the placement of the intravital microscope, as the probe and the electrode essentially need to occupy the same space in the hindlimb. With consideration of this problematic spacing, it may be useful to test the hypothesis that stimulation of the activating nerve induces more consistent muscle contractions than does direct stimulation of the muscle group. Should this hypothesis not be supported, suggesting that there is no benefit to direct nerve stimulation, then directly stimulating the muscle would alleviate some spacing issues as the electrodes could be placed at a position along the muscle farther from the muscular branch and the SDF intravital microscope.

To address other inconsistencies in the induced muscle contraction due to fatigue, it may be advantageous to consider the following options: only evaluate the microcirculation following stimulation at one frequency or increase the time allowed for recovery (6min), 
while decreasing the stimulation duration (3min). Based on subjective observation during experiments, the 3-minute stimulation time was unnecessary, as the muscular branch artery often reached its maximal diameter between 45 and 90 seconds. Thus reducing the 3-minute stimulation duration could ameliorate muscle fatigue.

\subsubsection{The Importance of Limb Order and Potential Considerations}

A final significant observation was that the order in which the limbs were imaged (first or second) had a significant effect on the vasodilatory response. The limb that was imaged first had a $78 \%$ increase in diameter compared to limbs imaged second, which had a $56 \%$ increase in diameter $(\mathrm{p}=0.006)$ (Figure 20). This was addressed during subsequent experiments by continuing to vary the order in which the ischemic and non-ischemic limbs were evaluated. However, this is not the optimal method for addressing this variance. It is assumed that this decrease in vascular function was due to low arterial pressure, which likely decreased during the experiment as a result of blood volume loss.

The oxygen gas used in combination with the inhalant anesthetic is very dry. Therefore, it is presumed that the animal likely lost blood volume due to excessive evaporation of extracellular water, subsequently inducing a decrease in arterial blood pressure and thus reduced hydrodynamic force to cause arterial distension. Previous studies have demonstrated that anesthesia induces a decrease in heart rate, blood pressure and tissue oxygen [66]. This issue has been addressed in other experiments involving prolonged anesthesia use, by carotid catheterization to monitor arterial blood pressure and address any significant changes [5]. 
With these considerations and variables withstanding, the protocol for evaluating vascular function using SDF imaging was suitable for subsequent experiments. Still, potential improvements can be made.

\subsection{Flow Control/Arterial Vasodilation During Remodeling of the Muscular} BRANCH ARTERY

Following verification of experimental protocols, the objective (as previously stated) was to examine vascular function following differing conditions of ischemia and remodeling. Thus, remodeling and then the collateral artery vasoactivity was evaluated with and without arteriogenesis (ligation and resection models respectively).

\subsubsection{Arteriogenesis}

As expected, the hindlimb ischemia caused by ligation of the femoral artery induced arteriogenesis of the muscular branch artery. Specifically, this collateralization was induced by the enhanced shear stress caused by the divergence of blood flow away from the superficial femoral artery and into the muscular branch and deep femoral arteries. This result is in support of previous research, which has demonstrated that in the same ligation model, collaterals in the gracilis muscle increased in diameter by $60 \%$ after 3 weeks [55]. In this experiment the muscular branch artery in the ischemic hindlimb was $17.4 \%$ larger than the same artery in the non-ischemic contralateral limb at rest after 2 weeks ( $\mathrm{p}=0.006)$. Further, the muscular branch artery in the ischemic hindlimb was $13.3 \%$ larger than the same artery in the non-ischemic contralateral limb following $8 \mathrm{~Hz}$ 
stimulation. It is expected that given another week for revascularization the artery may have expanded more, thought it would likely not experience the $60 \%$ increase in diameter observed in previous studies. However, the cited research that observed a $60 \%$ increase, examined smaller vessels within the gracilis compared to the large muscular branch arteriole, which likely normalized its response to elevated shear stress with a smaller percent increase in diameter [55].

\subsubsection{Vasodilation in Arteriogenic Conditions (Femoral Artery Ligation)}

Based on the aforementioned data it appears (based on previous research) that collateralization of the muscular branch was induced by the femoral artery ligation conditions (primarily high shear stress, normoxia and likely mild inflammation) [32]. Additionally it was expected that in this model, the conditions of high shear stress, would cause endothelial dysfunction and a loss of vasodilatory ability. While there appeared to be a trend towards impaired vascular function in the muscular branch artery, no significant $(\mathrm{p}>0.05)$ vasodilatory dysfunction was observed. This result was in contrast to what was hypothesized and previous research that demonstrated that hindlimb ischemia induced vascular dysfunction $[5,17]$. This previous research used a rat hindlimb ischemia model involving ligation of the common iliac artery; it was demonstrated that in the tibialis anterior (TA), 2 weeks after ligation, mild stimulation induced slight vasoconstriction in the muscle perfusing arterioles $[5,17]$. Further research demonstrated that in the extensor digitorum longus (EDL) arterioles constricted when exposed to acetylcholine (ACh), normally an agent that induces endothelial dependent vasodilation [5]. Another endothelial-dependent vasodilator, bradykinin, was also shown to have an 
impaired effect on the same vasculature following ligation [5]. Additionally it was demonstrated that endothelial-independent vasodilators also had an impaired effect on vascular function, but not until week 5 [5]. This seems to indicate that under ischemic conditions (following iliac artery ligation) in the lower limb endothelial regulation of vascular function is severely damaged as early as 2 weeks after ligation [5]. Other studies have confirmed the importance of endothelial integrity to vasoactivity, by damaging (through denudation) the inimal (EC) layer in the pig iliac artery and observing a constrictive response to ACh exposure (noramally a vasodilator) [67]. While it was hypothesized that similar vascular function impairment would occur in the collateral arteries of the thigh following femoral artery ligation (when elevated shear stress would cause endothelial deformation), this was not the case. Thus it is imperative to understand the differences in the tissue and blood flow conditions between the femoral artery ligation model, which this experiment used and the iliac ligation model that earlier experiments utilized.

\subsubsection{A Comparison of Hindlimb Ischemia Models to Clarify Vasodilation Results}

The most fundamental difference between these studies, but likely the most difficult to account for, was that the iliac ligation model was used in a rat and the femoral ligation model was used in a mouse. Thus, when considering differing results it is important to understand that even though both models were used in rodents, experimental conclusions may not always translate (similarly breakthroughs in animal disease models may not always work for humans or successful therapies may only help a subset of patients). With that in mind there are a number of other significant distinctions between each model. In 
the iliac artery ligation model resting blood flow to the entire hindlimb was reduced [5]. Specifically in the TA and EDL muscles, blood flow decreased by $40 \%$ [5]. These muscles also experienced significant hypoxia as a result of the insufficient blood flow [5, 17]. Gradual collateralization only induced a mild increase in blood flow by week 5 [5]. Thus, in the iliac artery ligation model it appeared that outward remodeling was an unlikely cause for the impairment of endothelial-dependent vascular control that occurred by week $2[5,17]$.

An important distinction between the vasculature studied by two models should be made, in that the muscular branch is a large arteriole, while the vessels examined in the previous models were smaller. Given that, it seems that the causes of endothelial-dependent vascular dysfunction in small, muscle perfusing vessels was not due to high blood flow, but instead factors that may include significant hypoxia and inflammation.

In the femoral artery ligation, however, it is expected that blood flow through the muscular branch artery is relatively undiminished. In fact, there should be an increase in blood flow and shear stress through the muscular branch artery as blood is diverged away from the occluded femoral artery. Blood perfusion through the ventral adductor muscle of the ischemic limb was approximately 1.3 and 1.5 times higher than the non-ischemic limb on days 3 and 7 respectively [55]. In the gracilis muscle, collateral diameter had increased $60 \%$ by week 3 [55]. Thus, collateralization was not gradual in the upper limb of the femoral artery ligation model as it was in the lower limb in the iliac artery ligation model. Further, hypoxia in the upper limb of the femoral artery ligation model was less 
severe than in the lower limb of the iliac ligation model or even the lower limb of the same model $[5,17,42]$.

Thus, based on the iliac artery ligation model hypoxia and low blood flow appear to be more likely causes for endothelial cell dysfunction compared to collateralization and high shear stress; therefore, the lack of vascular impairment that was observed in the femoral artery ligation model may have been expected. However, it is difficult to extend this conclusion necessarily to the larger arterioles examined in this mouse model. There are so many distinctions between these models that deducing the reason for the lack of vascular impairment requires more research. It could be that shear stress does not induce vascular dysfunction as speculated. However, it is possible that shear stress may induce dysfunction in smaller vessels like those evaluated in the iliac model rather than a vessel as large as the muscular branch. Perhaps low blood flow and its associated insults (hypoxia, nutrient shortage) are the primary cause for dysfunction and that is why in the femoral ligation model, where blood flow was high, impairment was not observed.

Inflammation severity may also play a role in dysfunction, and if so inflammation is expected to be higher in the lower leg where observations following iliac artery ligation were made. Further investigations will be required to continue to sort out the potential physiological causes for vascular dysfunction, but the observations of vascular remodeling and function following femoral artery resection may help further elucidate these factors. 


\subsubsection{Remodeling Following Non-Arteriogenic Ischemia}

In contrast to the femoral artery ligation model, the muscular branch artery in resected mice experienced minimal increases in shear stress and did not significantly outwardly remodel. Instead, there was neutral remodeling that occurred in this artery (Figure 24). The average resting diameter of the muscular branch artery was $63.3 \mu \mathrm{m}$ in the nonischemic contralateral hindlimb and $53.2 \mu \mathrm{m}$ in the ischemic hindlimb ( $>0.05)$. The average diameter for the muscular branch artery following $8 \mathrm{~Hz}$ stimulation was $113.1 \mu \mathrm{m}$ in the non-ischemic contralateral hindlimb and $105.3 \mu \mathrm{m}$ in the ischemic hindlimb $(p>0.05)$. Thus there appeared to be a slight but not significant decrease in the luminal diameter of the muscular branch following femoral artery resection and subsequent revascularization after 2 weeks. Previous studies have stressed the importance of shear stress in the induction of outward remodeling [30,32, 33]. This would imply that since there was neutral arterial remodeling that there was no increase in shear stress or blood flow following the ischemic insult. Based on observation, using the intravital microscope, blood flow through the muscular branch artery appeared particularly low/turbulent (qualitatively) (Figure 29). As stated previously, SDF imaging measures blood flow based on the contrast between dark hemoglobin containing red blood cells and white nonhemoglobin containing blood and tissue constituents. It was not possible to measure the blood flow through the muscular branch artery for two reasons: it was too large (diameter) and blood flow was too high. Thus, the contrast between black and white (red blood cells and plasma/white blood cells respectively) was not discernable. However, in some of the video samples taken with the SDF microscope of resected mice, contrast was distinguishable (Figure 28). Moreover, based on observation the contrast appeared to be 
swirling around and seemed to indicate that blood flow here was significantly lower and possibly turbulent. Previous research and the observation that this turbulent flow was predominantly directed from the gracilis to the superficial femoral artery (although it did appear to shift at times) indicate that blood flow to the gracilis vasculature and muscular branch artery, post resection, is being supplied by arteries such the medial circumflex, which connect with the muscular branch vasculature below the section of artery that was

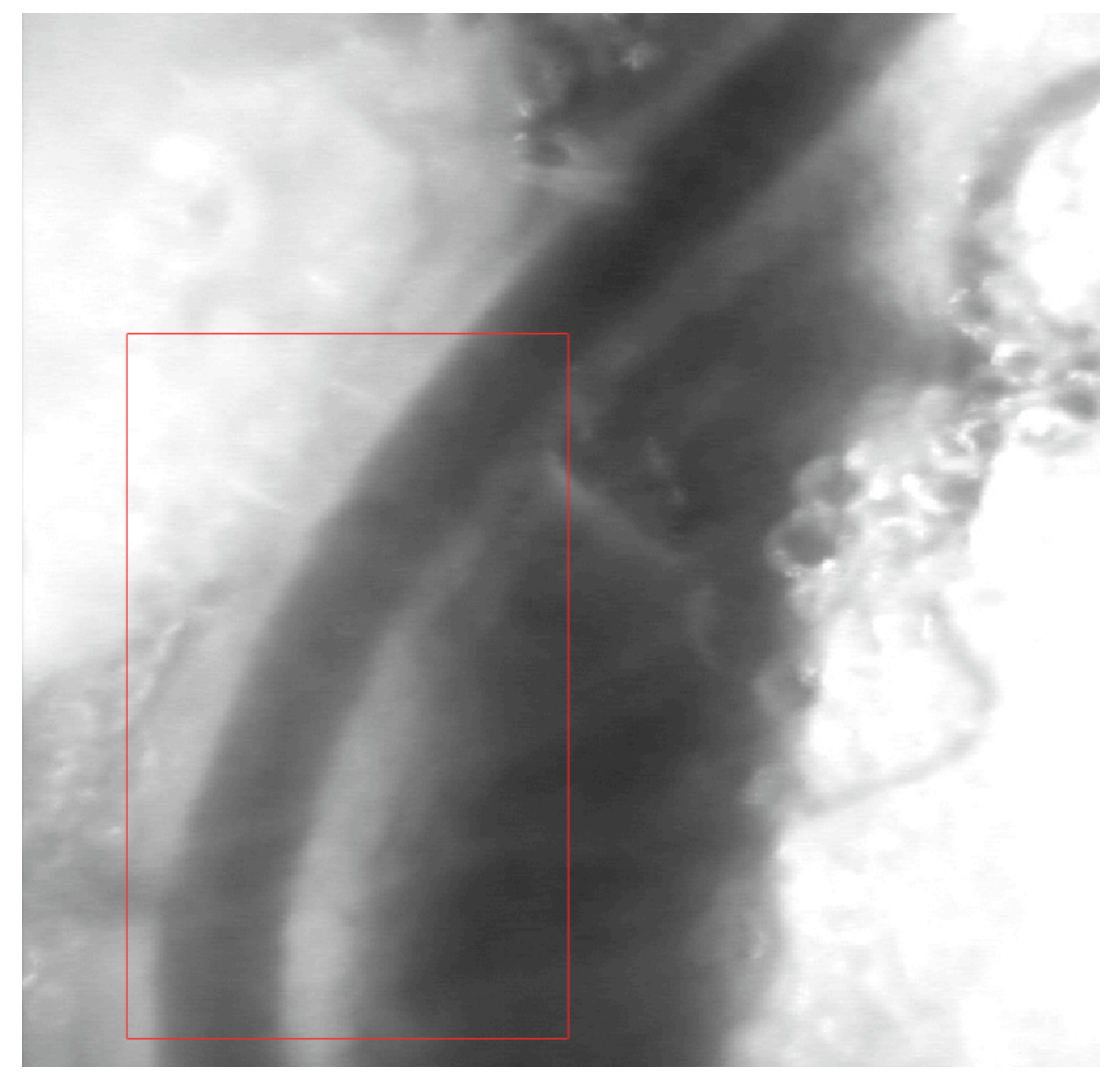

Figure 29. Image of turbulent flow in the muscular branch artery of resected mice. An SDF imaging snapshot of turbulent and low flow in the muscular branch artery. In the video it also appears that at times blood flow is moving up toward the cauterization site and thus not flowing to the gracilis muscle. Although during other time segments it seems as though blood flow may shift in the other direction.

imaged [58]. Thus, the low and turbulent flow occured likely because blood was having difficulty traveling past the muscular branch artery's now stump-like end with only small 
downstream vessels and high resistance (Figure 30). Both steep increases in resistance and obstructions (cauterized end of the larger muscular branch artery) are known to cause turbulent flow [68]. Interestingly, observations of the gracilis muscles in the hindlimb indicated that collateralization did occur throughout the vasculature of the muscle (Figure 31). This observation is consistent with previous data from a group that examined gracilis vasculature using the same model for hindlimb ischemia and found that the microcirculation of the ischemic gracilis muscles increased in diameter almost by a factor of 1.5 compared to the non-ischemic limb [18]. Thus, based on observation and previous data it appears that the impairment of vascular tone and potential decrease in shear stress may be exclusive to the muscular branch artery due to its abrupt increase in resistance following separation from the femoral artery blood supply during resection.

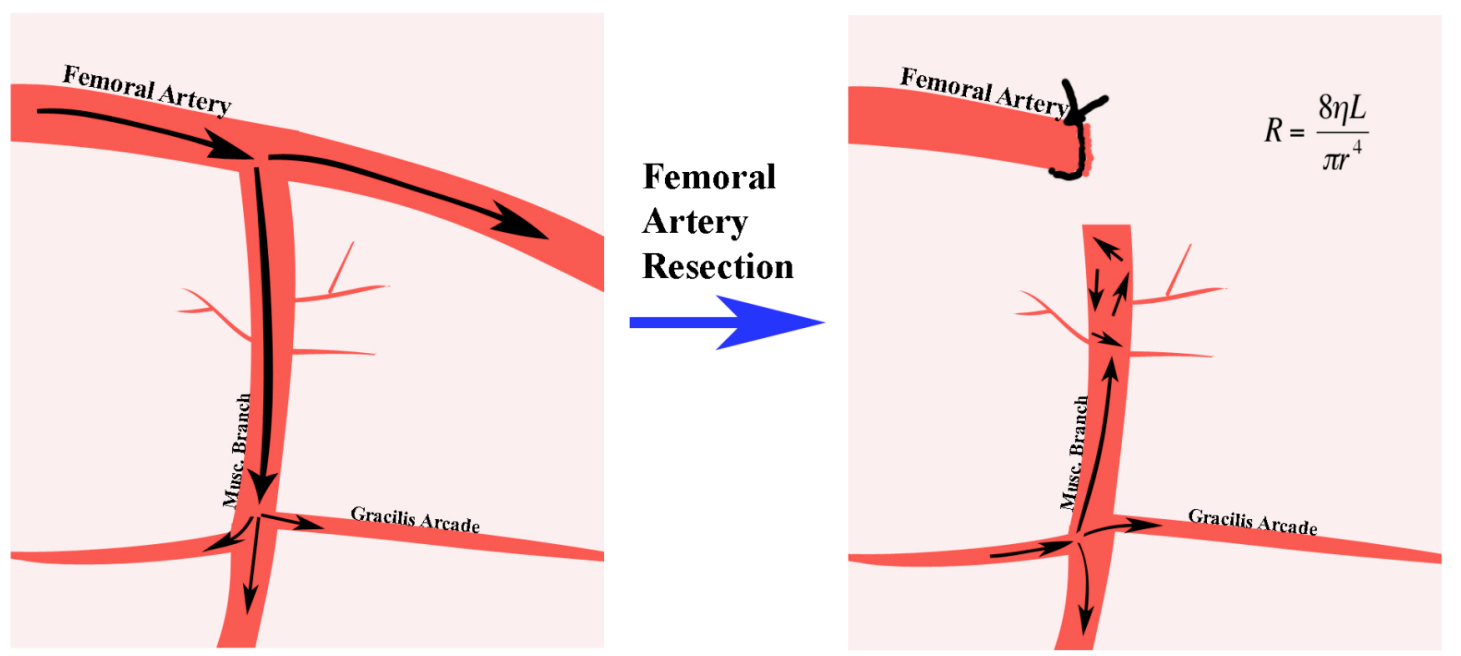

Figure 30. Blood flow changes following femoral artery resection. This diagram indicates how blood flow in the muscular branch artery becomes turbulent following femoral artery resection. Both the cauterized end of the muscular branch and the high resistance of small arteries connected to the muscular branch (recall that resistance is inversely related to the radius to the power of four) promote turbulent flow. 


\subsubsection{Vasodilation Following Non-Arteriogenic Ischemia}

Vasodilation in the muscular branch artery was slightly but not significantly enhanced in the resected ischemic compared to the non-ischemic hindlimb $(\mathrm{P}>0.05)$. In the resection model, no outward remodeling of the muscular branch artery occurred and the resting luminal diameter was slightly but not significantly smaller, as previously mentioned. Furthermore, the vessel was able to vasodilate to a luminal diameter not significantly

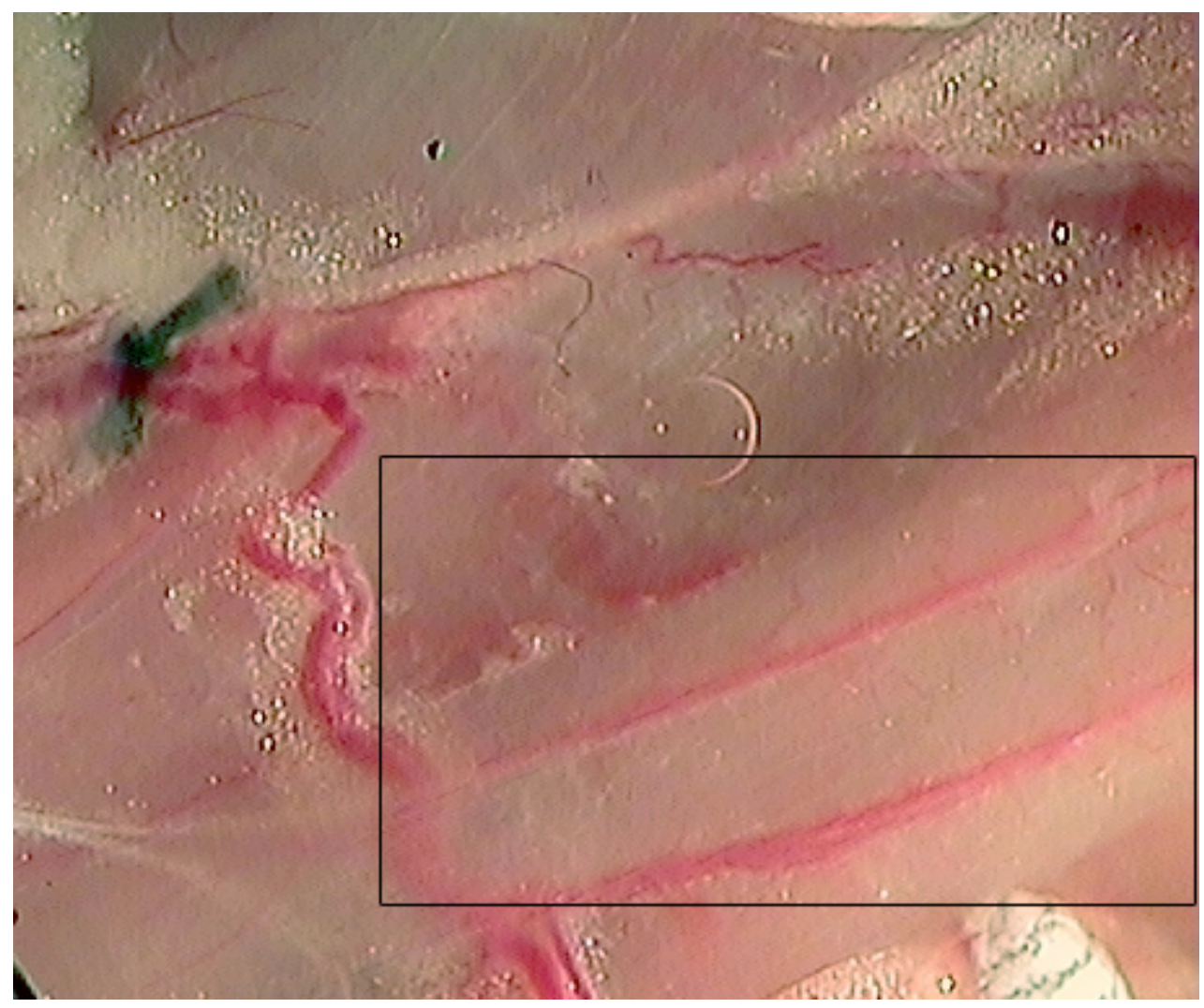

Figure 31. Remodeling of gracilis-perfusing vessels following femoral artery resection. Unfortunately no pictures happened to be taken of the contralateral non-ischemic hindlimb. However, based on observation these collaterals are significantly larger.

different than the non-ischemic artery. Thus it implies that the smaller resting diameter in the resection model yielded a slightly, but not significantly larger capacity for 
vasodilation; in the non-ischemic contralateral himdlimb the muscular branch artery dilated $79.3 \%$ following a stimulus frequency of $8 \mathrm{~Hz}$, while in the resected limb the same artery dilated $103.9 \%$ in response to an equivalent stimulation frequency ( $>0.05)$. While vasodilation was not impaired, it was clear through observation that the muscular branch artery in the resected limb had impaired ability to restore resting vascular tone. Following gracilis muscle contraction, the muscular branch artery required an excess of 30 minutes to even come close to the resting diameter. This was significantly larger when compared to the 5 minutes that were usually required for the same artery in sham and ligated mice to restore vascular tone. In a related but not identical response, an impairment of vasoconstriction was observed in the hindlimb of iliac artery ligated mice [5]. Following superfusion of noradrenaline and 5 weeks after iliac artery ligation arterioles in the EDL were not able to vasoconstrict effectively [5]. While the restoration response in the resection model is different, these observations do suggest that the tissue environment following resection may induce a related loss of vascular function. This is interesting in that our hypothesis that endothelial-deforming arteriogenesis would induce vascular dysfunction was unsupported, while non-arteriogenic factors such as inflammation and hypoxia may have induced blood flow control impairment similar to observations in the iliac artery ligation model. It is difficult to quantify this dysfunction, because no data was collected for the restoration of resting vascular tone. However, this is a potential area for future research of a unique aspect of vascular function. 


\subsection{ModificAtions For SubSequent Non-ARTERIOGENic Ischemia Models}

While this femoral artery resection model did generate some useful data, the observations of turbulent and low blood flow in the muscular branch artery demonstrate that blood flow through the muscular branch artery is not representative of blood flow through the gracilis vasculature (Figure 29, 30). Given this and some of the previously mentioned issues associated with analyzing the muscular branch artery following femoral artery resection, it would be potentially useful to identify new ways to analyze vascular function in vessels that do not undergo arteriogenesis following arterial occlusion with redirected blood flow after occlusion. One method would be to avoid the turbulence problems associated with the cauterization of the muscular branch artery and image the smaller vasculature that perfuses the gracilis muscle. Unfortunately, in the past, imaging these vessels using the SDF intravital microscope has proven difficult. Another potential option to better evaluate non-arteriogenic ischemia, given that it has been difficult to image gracilis vasculature, may be to use a different model or a slightly modified version of the resection model of hindlimb ischemia. One option would be to use the previously discussed model used to demonstrate impaired vascular function following chronic ischemia in the lower limb (EDL). This rat model involved the ligation of the common iliac artery, which significantly reduced blood flow in the hindlimb, as the common iliac artery serves as the main feed vessel for the hindlimb (recall circulation from Figure 8) $[69,70]$. In this model the muscular branch artery would be exposed to non-arteriogenic conditions, as the vessel does not experience increased blood flow following common iliac artery ligation. Importantly, blood flow through the muscular branch would not be abruptly occluded (no cauterization required) and velocity through that vessel would be 
consistent with the vasculature in the gracilis muscle. Thus, SDF imaging could be used to evaluate the only artery in the ischemic hindlimb that has been successfully visualized and examined. Another potential model, which appears to not have been used as of yet, would actually be a modification of the current resection model of hindlimb ischemia. This model would involve the resection of the femoral artery vein pair from near the saphenous bifurcation to just distally of the deep femoral artery (Figure 32). Then the femoral artery vein pair would be ligated proximal to the muscular branch artery (Figure 32). This model would be nearly identical to the current resection model, except blood would be able to flow from the muscular branch artery to the deep femoral artery. Thus, there would be no abrupt resistance increase to the muscular branch artery and theoretically blood flow would be more representative of that through the vasculature of the ischemic hindlimb and specifically the gracilis muscles.

These models could also be used to examine what factors, other than shear stress, induce arteriogenesis. Previous research has demonstrated that enhanced shear stress is the driving factor for arteriogenesis [32]. However, as stated above, in this low shear stress 


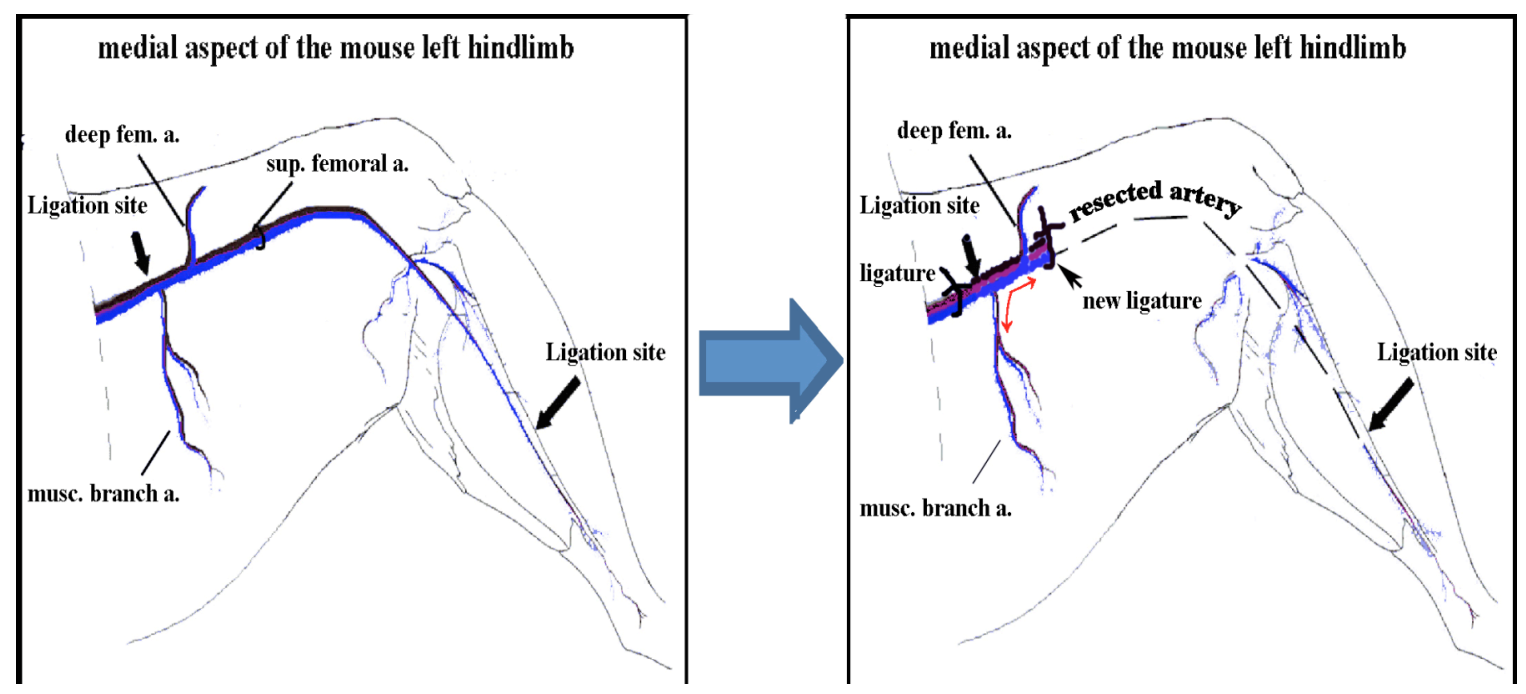

Figure 32. A modified femoral artery resection model. This surgery would allow for blood flow to flow through the muscular branch artery without high resistance following occlusion, yet still keep the vessel "out of series" with the diverged blood flow.

resection model, significant collateralization was still observed throughout the gracilis (Figure 31). Even more, this outward remodeling often seemed more ubiquitous and extensive than in the ligation model of ischemia (no pictures taken, only subjectively observed). Understanding what conditions led to this significant collateralization would be useful to understanding revascularization.

\subsection{FUtURE INVESTIGATION}

It remains unclear what specific conditions or processes impair vascular function in chronic ischemia. Based on this investigation and previous work it appears that collateralization and increased shear stress do not effectuate vascular function impairment in arterioles. However, to definitively make this conclusion and identify the cause of vascular dysfunction following chronic ischemia, it would be useful to examine 
conditions of increased shear stress in different arterial types. Furthermore, it would be of interest to get a more direct measure of arterial blood flow, either by developing a protocol to observe smaller vasculature where SDF imaging is capable of measuring blood flow or by taking blood pressure measurements (combining these with luminal diameter measurements). It may also be useful to use the proposed modified resection model when examining the muscular branch artery, so that the resistance to flow in this artery is not abnormally high. A revised resection model to ensure accurate evaluation of the muscular branch artery may result in different and interesting results. Finally, it may be useful to examine vascular function in the lower hindlimb, where ischemia, hypoxia and inflammation are more severe, in order to compare this with data acquired in the upper hindlimb. This comparison would carry more validity than that made between the ligation model and the iliac model previously discussed because the same model would be used in the same species (rather than rat vs. mouse). Ultimately, the identification of which factors (shear stress, hypoxia, inflammation, etc.) cause vascular dysfunction would be useful for application in PAD. Patients who experience intermittent claudication and have difficulty exercising, even if they have gone through successful revascularization (surgical or therapeutic) would greatly benefit from therapies addressing impaired blood flow control, so that they can exercise and work to reverse the progression of this disease. 


\section{REFERENCES}

1. Stein, J.H., ed. Internal Medicine, Stein - 5th Ed. Internal Medicine. 1998, Mosby, Inc.

2. Johnson, L.R. and B.J. H., Essential Medical Physiology. 2003, San Diego: Academic Press.

3. Dietrich, H.H., et al., Red blood cell regulation of microvascular tone through adenosine triphosphate. Am J Physiol Heart Circ Physiol, 2000. 278(4): p. H1294-8.

4. Duza, T. and I.H. Sarelius, Conducted dilations initiated by purines in arterioles are endothelium dependent and require endothelial Ca2+. Am J Physiol Heart Circ Physiol, 2003. 285(1): p. H26-37.

5. Kelsall, C.J., M.D. Brown, and O. Hudlicka, Alterations in reactivity of small arterioles in rat skeletal muscle as a result of chronic ischaemia. J Vasc Res, 2001. 38(3): p. 212-8.

6. Rhoades, R.A. and D.R. Bell, eds. Medical Physiology. 3rd ed. 2009, Lippincott Williams \& Wilkins: China.

7. Ganong, W.F., Review of Medical Physiology. 2003, New York: McGraw-Hill.

8. Human Body: Circulatory System. 2007, Dorling Kindersley.

9. Casiday, R. and R. Frey. Hemeglobin and the Heme Group: Metal Complexes in the Blood for Oxygen Transport. Inorganic Synthesis Experiment 1998 [cited Dec. 1, 2008]; Available from: http://www.chemistry.wustl.edu/ courses/genchem/Tutorials/Hemoglobin/151_T 3 hemoglobin.htm. 
10. GCSE Science/The Heart. [cited Dec. 1, 2008]; Available from: http://en.wikibooks.org/wiki/GCSE_Science/The_Heart.

11. Freudenrich, C. How Your Lungs Work. How Stuff Works 2000 [cited Dec. 1, 2008]; Available from: http://health.howstuffworks.com/lung2.htm.

12. Internal and External Respiration. [cited Dec. 1, 2008]; Available from: http://www.coolschool.ca/lor/BI12/unit11/U11L05.htm.

13. Guyton, A.C. and J.E. Hall, Textbook of Medical Physiology 11th Ed. 2006: Elsevier Inc.

14. van Weel, V., et al., Vascular growth in ischemic limbs: a review of mechanisms and possible therapeutic stimulation. Ann Vasc Surg, 2008. 22(4): p. 582-97.

15. Shamoun, F., N. Sural, and G. Abela, Peripheral artery disease: therapeutic advances. Expert Rev Cardiovasc Ther, 2008. 6(4): p. 539-53.

16. Humes, H.D., et al., eds. Kelley's Textbook of Internal Medicine, 4th Ed. 2000, Lippincott Williams \& Wilkins: Philadelphia.

17. Hudlicka, O., et al., Effect of long-term electrical stimulation on vascular supply and fatigue in chronically ischemic muscles. J Appl Physiol, 1994. 77(3): p. 131724.

18. He, Y., et al., Critical function of Bmx/Etk in ischemia-mediated arteriogenesis and angiogenesis. J Clin Invest, 2006. 116(9): p. 2344-55.

19. Halperin, J.L., Evaluation of patients with peripheral vascular disease. Thromb Res, 2002. 106(6): p. V303-11.

20. Braun, L.T., Cardiovascular disease: strategies for risk assessment and modification. J Cardiovasc Nurs, 2006. 21(6 Suppl 1): p. S20-42; quiz S43-5. 
21. Ismail, H.M., K. Jackson, and D. Smith, Diagnosis and treatment of peripheral arterial disease compared with other atherosclerotic vascular diseases in a university primary care clinic. J Investig Med, 2006. 54(5): p. 255-61.

22. Novo, S., G. Coppola, and G. Milio, Critical limb ischemia: definition and natural history. Curr Drug Targets Cardiovasc Haematol Disord, 2004. 4(3): p. 219-25.

23. Berger, A. Developmental process of atherosclerosis. 2008 [cited 2008; Available from:

http://www.nlm.nih.gov/medlineplus/ency/imagepages/18020.htm.

24. Dagher, N.N. and J.G. Modrall, Pharmacotherapy before and after revascularization: anticoagulation, antiplatelet agents, and statins. Semin Vasc Surg, 2007. 20(1): p. 10-4.

25. Nikol, S., Therapeutic angiogenesis for peripheral artery disease: gene therapy. Vasa, 2007. 36(3): p. 165-73.

26. De Sanctis, J.T., Percutaneous interventions for lower extremity peripheral vascular disease. Am Fam Physician, 2001. 64(12): p. 1965-72.

27. Taniyama, Y., et al., Therapeutic angiogenesis induced by human hepatocyte growth factor gene in rat and rabbit hindlimb ischemia models: preclinical study for treatment of peripheral arterial disease. Gene Ther, 2001. 8(3): p. 181-9.

28. Merriam-Webster's Medical Dictionary. 2009 [cited Dec. 1, 2008]; Available from: http://www2.merriam-webster.com/cgi$\underline{\text { bin } / \text { mwmedsamp?book=Medical } \& v a=\text { sample. }}$.

29. Luttun, A., et al., Revascularization of ischemic tissues by PlGF treatment, and inhibition of tumor angiogenesis, arthritis and atherosclerosis by anti-Flt1. Nat Med, 2002. 8(8): p. 831-40. 
30. Cai, W. and W. Schaper, Mechanisms of arteriogenesis. Acta Biochim Biophys Sin (Shanghai), 2008. 40(8): p. 681-92.

31. Schaper, W. and I. Buschmann, Arteriogenesis, the good and bad of it. Cardiovasc Res, 1999. 43(4): p. 835-7.

32. Scholz, D., et al., Contribution of arteriogenesis and angiogenesis to postocclusive hindlimb perfusion in mice. J Mol Cell Cardiol, 2002. 34(7): p. 77587.

33. Scholz, D., et al., Ultrastructure and molecular histology of rabbit hind-limb collateral artery growth (arteriogenesis). Virchows Arch, 2000. 436(3): p. 25770.

34. Blue Histology - Vascular System. 2006 [cited Dec. 1, 2008]; Available from: http://www.lab.anhb.uwa.edu.au/mb140/CorePages/Vascular/Vascular.htm.

35. Wahlberg, E. Angiogenesis and Arteriogenesis - the Basics. 2008 [cited Dec. 1, 2008]; Available from:

http://www.vascularweb.org/professionals/Research/Basic_Articles/Angiogenesis Wahlberg.html.

36. Arras, M., et al., Monocyte activation in angiogenesis and collateral growth in the rabbit hindlimb. J Clin Invest, 1998. 101(1): p. 40-50.

37. Pasyk, S., et al., DNA synthesis in coronary collaterals after coronary artery occlusion in conscious dog. Am J Physiol, 1982. 242(6): p. H1031-7.

38. Helisch, A. and W. Schaper, Arteriogenesis: the development and growth of collateral arteries. Microcirculation, 2003. 10(1): p. 83-97.

39. Brevetti, L.S., et al., Overexpression of endothelial nitric oxide synthase increases skeletal muscle blood flow and oxygenation in severe rat hind limb ischemia. J Vasc Surg, 2003. 38(4): p. 820-6. 
40. Cai, W.J., et al., Expression of endothelial nitric oxide synthase in the vascular wall during arteriogenesis. Mol Cell Biochem, 2004. 264(1-2): p. 193-200.

41. Clayton, J.A., D. Chalothorn, and J.E. Faber, Vascular endothelial growth factorA specifies formation of native collaterals and regulates collateral growth in ischemia. Circ Res, 2008. 103(9): p. 1027-36.

42. Mees, B., et al., Endothelial nitric oxide synthase activity is essential for vasodilation during blood flow recovery but not for arteriogenesis. Arterioscler Thromb Vasc Biol, 2007. 27(9): p. 1926-33.

43. Ho, Q.T. and C.J. Kuo, Vascular endothelial growth factor: biology and therapeutic applications. Int J Biochem Cell Biol, 2007. 39(7-8): p. 1349-57.

44. Couffinhal, T., et al., Mouse model of angiogenesis. Am J Pathol, 1998. 152(6): p. $1667-79$.

45. Cao, Y., et al., In vivo angiogenic activity and hypoxia induction of heterodimers of placenta growth factor/vascular endothelial growth factor. J Clin Invest, 1996. 98(11): p. 2507-11.

46. Yu, J., et al., Endothelial nitric oxide synthase is critical for ischemic remodeling, mural cell recruitment, and blood flow reserve. Proc Natl Acad Sci U S A, 2005. 102(31): p. 10999-1004.

47. Lee, J., et al., Vascular endothelial growth factor-related protein: a ligand and specific activator of the tyrosine kinase receptor Flt4. Proc Natl Acad Sci U S A, 1996. 93(5): p. 1988-92.

48. Takeshita, S., et al., Therapeutic angiogenesis. A single intraarterial bolus of vascular endothelial growth factor augments revascularization in a rabbit ischemic hind limb model. J Clin Invest, 1994. 93(2): p. 662-70. 
49. Witzenbichler, B., et al., Vascular endothelial growth factor-C (VEGF-C/VEGF2) promotes angiogenesis in the setting of tissue ischemia. Am J Pathol, 1998. 153(2): p. 381-94.

50. Tissue Growth and Repair. [cited Dec. 1, 2008]; Available from: http://www.gene.com/gene/research/focusareas/tgr/.

51. Folkman, J., Angiogenesis in cancer, vascular, rheumatoid and other disease. Nat Med, 1995. 1(1): p. 27-31.

52. Plate, K.H., et al., Up-regulation of vascular endothelial growth factor and its cognate receptors in a rat glioma model of tumor angiogenesis. Cancer Res, 1993. 53(23): p. 5822-7.

53. Jackson, W.F., Potassium channels in the peripheral microcirculation. Microcirculation, 2005. 12(1): p. 113-27.

54. Dormandy, J., L. Heeck, and S. Vig, The fate of patients with critical leg ischemia. Semin Vasc Surg, 1999. 12(2): p. 142-7.

55. Chalothorn, D., et al., Catecholamines augment collateral vessel growth and angiogenesis in hindlimb ischemia. Am J Physiol Heart Circ Physiol, 2005. 289(2): p. H947-59.

56. Chalothorn, D., et al., Heparin-binding epidermal growth factor-like growth factor, collateral vessel development, and angiogenesis in skeletal muscle ischemia. Arterioscler Thromb Vasc Biol, 2005. 25(9): p. 1884-90.

57. Ryzhov, S., et al., Role of adenosine receptors in the regulation of angiogenic factors and neovascularization in hypoxia. J Pharmacol Exp Ther, 2007. 320(2): p. $565-72$. 
58. Sullivan, C.J., T. Doetschman, and J.B. Hoying, Targeted disruption of the Fgf2 gene does not affect vascular growth in the mouse ischemic hindlimb. J Appl Physiol, 2002. 93(6): p. 2009-17.

59. Goedhart, P., et al., Sidestream Dark Field (SDF) imaging: a novel stroboscopic LED ring-based imaging modality for clinical assessment of the microcirculation. Optics Express, 2007. 15(23): p. 15101-15114.

60. Dondorp, A.M., et al., Direct in vivo assessment of microcirculatory dysfunction in severe falciparum malaria. J Infect Dis, 2008. 197(1): p. 79-84.

61. Nieuwdorp, M., et al., Measuring endothelial glycocalyx dimensions in humans: a potential novel tool to monitor vascular vulnerability. J Appl Physiol, 2008. 104(3): p. 845-52.

62. Bezemer, R., et al., Sidestream dark-field imaging versus orthogonal polarization spectroscopic imaging: a comparative study. Critical Care, 2008. 12: p. P63.

63. Dobbe, J.G.G., Automated Vascular Analysis. 2007, Academic Medical Center, University of Amsterdam: Amsterdam.

64. Proctor, K.G. and S.L. Bealer, Skeletal muscle vasodilation during electrical stimulation of the preoptic recess. Am J Physiol, 1986. 250(2 Pt 2): p. H221-5.

65. Gorczynski, R.J., B. Klitzman, and B.R. Duling, Interrelations between contracting striated muscle and precapillary microvessels. Am J Physiol, 1978. 235(5): p. H494-504.

66. Hirata, K. and T. Nagasaka, Calorigenic and cardiovascular responses to norepinephrine in anesthetized and unanesthetized control and cold-acclimated rats. Jpn J Physiol, 1981. 31(3): p. 305-16.

67. Ohwada, T., et al., Specificity of vascular reactivity and remodeling after repeated endothelial injury in a swine model. Int Heart J, 2006. 47(2): p. 297-310. 
68. Batchelor, G.K., An Introduction to Fluid Mechanics. 2000, New York:

Cambridge University Press.

69. Brown, M.D., et al., Remodeling in the microcirculation of rat skeletal muscle during chronic ischemia. Microcirculation, 2003. 10(2): p. 179-91.

70. Hudlicka, O. and S. Price, The role of blood flow and/or muscle hypoxia in capillary growth in chronically stimulated fast muscles. Pflugers Arch, 1990. 417(1): p. 67-72. 


\section{APPENDIX A}

\section{VASCULAR FUNCTION AND BLOOD FLOW EQUATIONS}

The relation between arterial diameter and blood flow can be described using an extension of Ohm's Law for circuits which describes the relationship between electrical current (I), applied electromotive force (E) and resistance (R), and is analogous to the relationship between blood flow $(\mathrm{F})$, blood pressure $(\mathrm{P})$ and vessel resistance (Equation 1a and $1 \mathrm{~b}$ respectively). Blood vessel resistance can be related to fluid viscosity ( $)$, vessel length (L) and vessel radius (r, Equation 1c). Combining these equations (with the knowledge that vessel pressure equals the pressure difference between vessel endpoints, $\mathrm{P}_{\mathrm{A}}-\mathrm{P}_{\mathrm{B}}$ ) yields the Poisseuille-Hagen formula (Equation 2).

a)

$$
I=\frac{E}{R}
$$

b)

$$
F=\frac{P}{R}
$$

c)

$$
R=\frac{8 \eta L}{\pi r^{4}}
$$

Equation 2. a) Ohm's Law for an electrical circuit. b) Ohm's Law as it applies to blood flow. c) The equation describing blood flow resistance (adapted from [12]).

$$
F=\frac{\Delta P}{R}=\frac{\left(P_{A}-P_{B}\right) \pi r^{4}}{8 \eta L}
$$

Equation 3. The Poisseuille-Hagen formula, a combination of the formulas from Equation 1 (adapted from [12]). 


\title{
APPENDIX B
}

\author{
HUSBANDRY PRACTICES
}

\section{Daily}

1. Prior to entering room, apply booties \& disinfect booties with Clidox. If changing cages, apply bonnet, isolation mask, and isolation gown.

2. Record room temperature

3. Check cleanliness of water, which should not be cloudy or opaque

4. Ensure adequate levels of feed \& water

5. Perform cage census

6. Observe behavior within cage

a. On cage activity form, record unusual injury*, illness**, and fighting

b. If animals are found dead or moribund (near death), contact Trevor Cardinal (6-6244, 503-9837)

7. Record notes and activities in notebook

\section{Weekly (or more frequently if needed***)}

1. Complete all steps outlined in Daily Husbandry Practices

2. To a clean cage apply:

a. Bedding

b. Clean mouse house

c. Clean wire lid

i. Within wire lid place:

1. Feed

2. Clean water bottle containing distilled water

d. Clean microisolator lid with a fresh filter

3. Weigh mice and record by ear-tag number

4. Transfer mice to the clean cage

5. Transfer cage card to the clean cage

6. Transport soiled cages outside the room to sink or dishwasher (using sanitize cycle)
a. Hand-wash cages and cage accessories with Liquinox detergent
b. Rinse with Clidox disinfectant
c. Rinse with ultra-purified water
d. Place cages on bench cover, on cage rack and allow to air dry
e. Once dry, reassemble cages with a new filter and leave on cage rack 


\section{APPENDIX C}

\section{SDF IMAGING EXPERIMENTAL CHECKLIST}

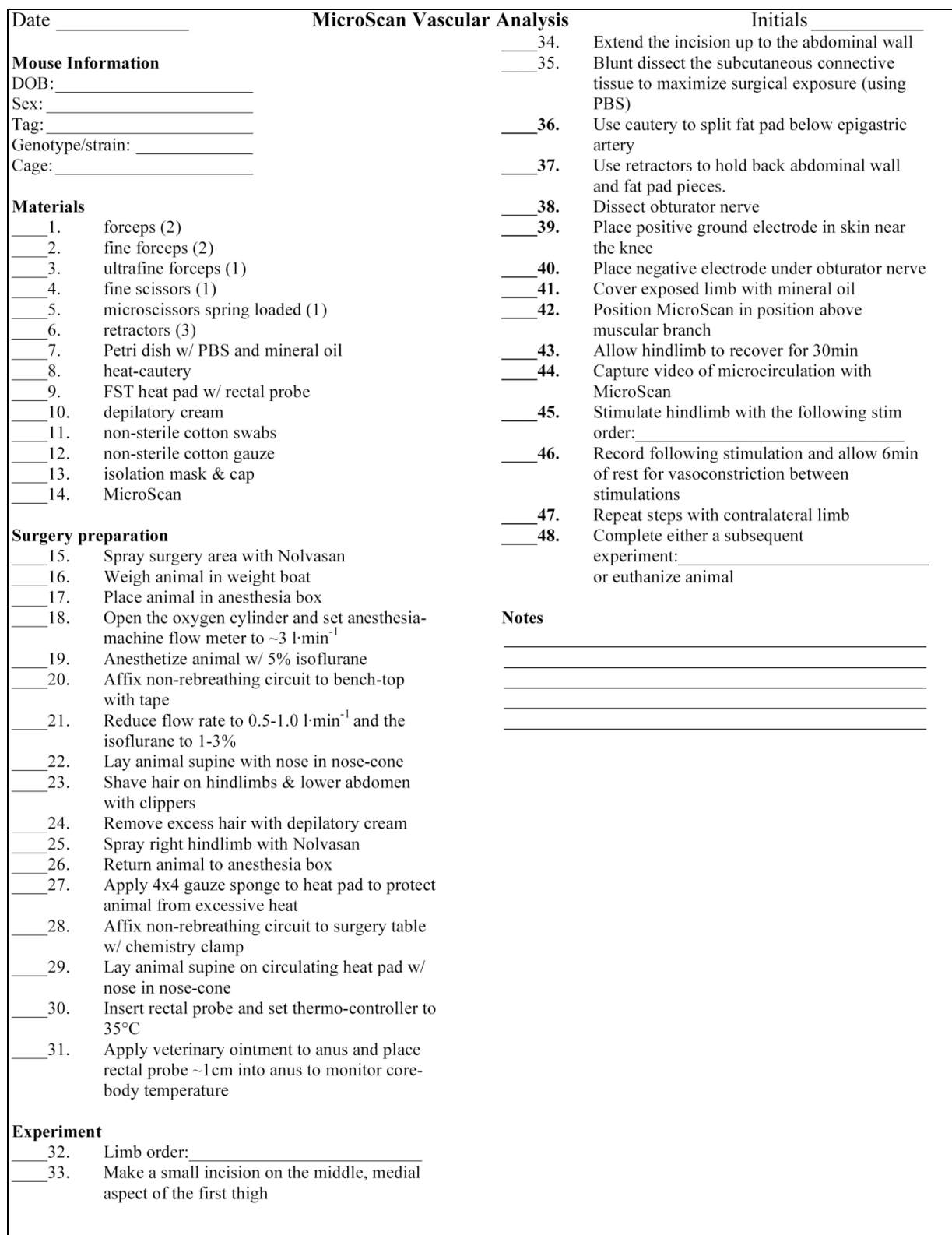

Figure 33. SDF imaging experimental checklist. This checklist was used when recording muscular branch artery functional hyperemia in healthy and ischemic mice. 


\section{APPENDIX D}

\section{Femoral ARTERy Ligation CHeCKList}

\begin{tabular}{|c|c|c|c|}
\hline \multicolumn{4}{|c|}{ Hindlimb Ischemia Surgery - Ligation } \\
\hline \multicolumn{4}{|c|}{ Mouse Information } \\
\hline \multicolumn{2}{|c|}{ DOB: } & 35. & Apply $4 \times 4$ gauze sponge to heat pad to \\
\hline \multicolumn{2}{|l|}{ Sex: } & & protect animal from excessive heat \\
\hline \multicolumn{2}{|l|}{ Tag: } & 36. & Affix non-rebreathing circuit to surgery \\
\hline \multicolumn{2}{|c|}{$\begin{array}{l}\text { Genotype/strain: } \\
\text { Cage: }\end{array}$} & & table w/ chemistry clamp \\
\hline & 37. & $\begin{array}{l}\text { Lay animal supine on circulating heat pad } \\
\text { w/ nose in nose-cone }\end{array}$ \\
\hline \multicolumn{2}{|c|}{ Materials } & 38. & Insert rectal probe and set thermo-controller \\
\hline \multicolumn{2}{|c|}{ Sterilize- autoclave or flash autoclave } & & to $37^{\circ} \mathrm{C}$ \\
\hline 1. & forceps (2) & 39. & Apply veterinary ointment to eyes to avoid \\
\hline 2. & fine forceps (2) & & drying during procedure \\
\hline 3. & ultrafine forceps (1) & 40 . & Apply veterinary ointment to anus and place \\
\hline 4. & fine scissors (1) & & rectal probe $\sim 1 \mathrm{~cm}$ into anus to monitor core- \\
\hline 5 . & microscissors spring loaded (1) & & body temperature \\
\hline \multicolumn{2}{|c|}{ Pre-sterilize in autoclave } & Surgery & \\
\hline 6. & cotton gauze (2) & 41. & Make a small incision on the middle, medial \\
\hline 7. & cotton swabs (12) & & aspect of the left thigh \\
\hline 8. & 6.0 silk suture $(2 \times 1$-inch $)$ & 42. & Extend the incision up to the abdominal wall \\
\hline 9. & needle holder (1) & 43. & Blunt dissect the subcutaneous connective \\
\hline \multicolumn{2}{|c|}{ Obtained in surgery suite } & & $\begin{array}{l}\text { tissue and fat pad to maximize surgical } \\
\text { exposure }\end{array}$ \\
\hline & sterile Petri dish w/ sterile saline & 44. & Blunt dissect the femoral artery from the \\
\hline \multicolumn{2}{|c|}{$\begin{array}{ll}\text { 10. } \\
\text { 11. } \\
\text { 11. } \\
\text { sterile gloves }\end{array}$} & & neurovascular bundle just downstream from \\
\hline \multicolumn{2}{|c|}{ 12. sterile 7.0 prolene suture } & & the deep femoral branch \\
\hline \multirow{2}{*}{\multicolumn{2}{|c|}{$\begin{array}{ll}\text { 13. } & \text { heat-cautery } \\
& \text { 14. }\end{array}$}} & 45. & Tie off the femoral artery \& vein with 6.0 \\
\hline & & & silk suture, just downstream to the deep \\
\hline 15 & heat pad & & femoral branch \\
\hline \multirow{7}{*}{$\begin{array}{r}16 . \\
17 . \\
18 . \\
19 . \\
20 . \\
21 .\end{array}$} & recovery bin \& weigh boat & 46. & Use 7.0 polypropylene suture to close the \\
\hline & depilatory cream & & skin \\
\hline & non-sterile cotton swabs & 47. & Make a small incision on the middle, medial \\
\hline & non-sterile cotton gauze & & aspect of the right thing \\
\hline & isolation mask \& cap & 48. & Extend the incision up to the abdominal wall \\
\hline & analgesic (Buprenorphine) & 49. & Blunt dissect the subcutaneous connective \\
\hline & & & tissue to maximize surgical exposure \\
\hline \multicolumn{2}{|c|}{ Surgery preparation } & 50 . & Use 7.0 polypropylene suture to close the \\
\hline 22. & Spray surgery area with Nolvasan & & skin \\
\hline & Weigh animal in weight boat & & \\
\hline 24. & Place animal in anesthesia box & Post-Sur & \\
\hline 25 . & $\begin{array}{l}\text { Open the oxygen cylinder and set } \\
\text { anesthesia-machine flow meter to } \sim 3 \mathrm{l} \cdot \mathrm{min}^{-1}\end{array}$ & & $\begin{array}{l}\text { Give the animal an subcutaneous injection } \\
\text { of buprenorphine }(0.075 \mathrm{mg} / \mathrm{kg})\end{array}$ \\
\hline 26. & Anesthetize animal w/ 5\% isoflurane & 52. & Place the animal in the recovery bin, on a \\
\hline 27. & $\begin{array}{l}\text { Affix non-rebreathing circuit to bench-top } \\
\text { with tape }\end{array}$ & & $\begin{array}{l}\text { blue bench cover, above a heat pad and } \\
\text { allow to recover }\end{array}$ \\
\hline 28. & $\begin{array}{l}\text { Reduce flow rate to } 0.5-1.01 \cdot \mathrm{min}^{-1} \text { and the } \\
\text { isoflurane to } 1-3 \%\end{array}$ & & $\begin{array}{l}\text { Turn flow meter down to } 0 \text {, turn off } \\
\text { isoflurane, and close the oxygen cylinder }\end{array}$ \\
\hline 29. & Apply ear tag high on left ear & 54. & Indicate surgery on cage card \\
\hline & Lay animal supine with nose in nose-cone & & \\
\hline 31. & $\begin{array}{l}\text { Shave hair on the right hindlimb \& lower } \\
\text { abdomen with clippers }\end{array}$ & Notes & \\
\hline 32. & Remove excess hair with depilatory cream & & \\
\hline 33. & Spray right hindlimb with Nolvasan & & \\
\hline & Return animal to anesthesia box & & \\
\hline
\end{tabular}

Figure 34. Femoral Artery Ligation Checklist. Checklist used during femoral artery ligation surgeries. 


\section{APPENDIX E}

\section{FEMORAL ARTERY ReSECTION CHECKLIST}

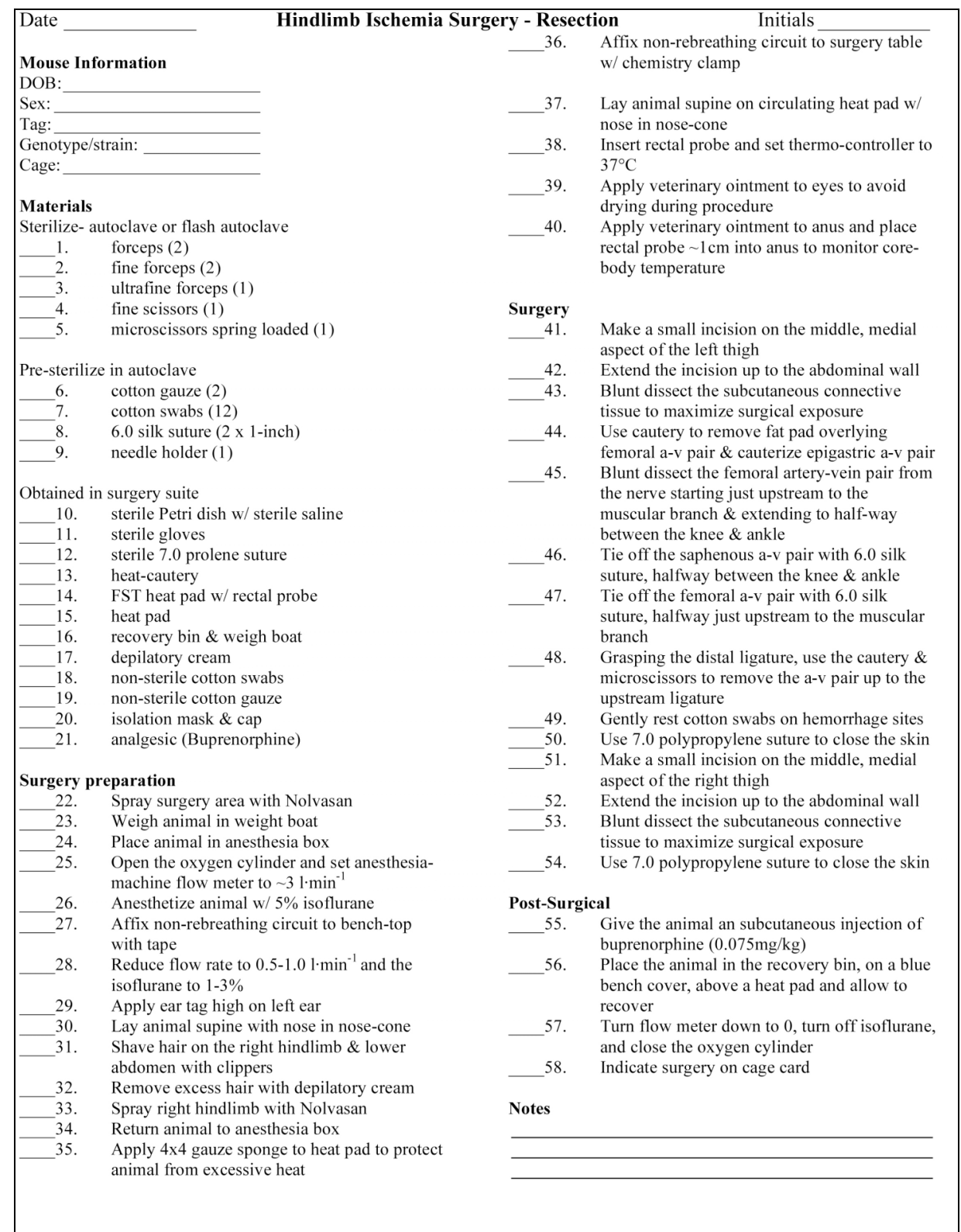

Figure 35. Femoral Artery Resection Checklist. Checklist used during femoral artery resection surgeries. 


\section{APPENDIX F}

\section{Muscular Branch Functional Hyperemia Data for Healthy Mice}

Table 1. Muscular branch functional hyperemia data for healthy mice.

\begin{tabular}{|c|c|c|c|c|}
\hline Mouse & Order Imaged & Limb & $\begin{array}{c}\text { Stimulation } \\
\text { Frequency }(\mathrm{Hz})\end{array}$ & $\begin{array}{c}\text { Muscular Branch } \\
\text { Arterial Diameter }(\boldsymbol{\mu} \mathrm{m})\end{array}$ \\
\hline 1 & First & Left & 0 & 59.637 \\
\hline & Second & Right & 0 & 63.503 \\
\hline 2 & First & Right & 0 & 53.277 \\
\hline 2 & Second & Left & 0 & 74.71 \\
\hline & First & Right & 0 & 63.07 \\
\hline & Second & Left & 0 & 63.53 \\
\hline 4 & First & Left & 0 & 64.91 \\
\hline 4 & Second & Right & 0 & 69.68 \\
\hline 5 & First & Right & 0 & 68.12 \\
\hline 5 & Second & Left & 0 & 68.02 \\
\hline 1 & First & Left & 2 & 91.36 \\
\hline 1 & Second & Right & 2 & 81.53 \\
\hline & First & Right & 2 & 84.3 \\
\hline & Second & Left & 2 & 87.3 \\
\hline 3 & First & Right & 2 & 93.56 \\
\hline 3 & Second & Left & 2 & 97.67 \\
\hline & First & Left & 2 & 112.62 \\
\hline 4 & Second & Right & 2 & 90.69 \\
\hline 5 & First & Right & 2 & 91.75 \\
\hline 5 & Second & Left & 2 & 82.2 \\
\hline 1 & First & Left & 4 & 122.66 \\
\hline 1 & Second & Right & 4 & 90.74 \\
\hline 2 & First & Right & 4 & 97.37 \\
\hline & Second & Left & 4 & 97.95 \\
\hline & First & Right & 4 & 109.45 \\
\hline & Second & Left & 4 & 131.25 \\
\hline 4 & First & Left & 4 & 136.62 \\
\hline 4 & Second & Right & 4 & 105.71 \\
\hline 5 & First & Right & 4 & 127.36 \\
\hline 5 & Second & Left & 4 & 107.15 \\
\hline 1 & First & Left & 8 & 118.373 \\
\hline 1 & Second & Right & 8 & 110.443 \\
\hline 2 & First & Right & 8 & 100.55 \\
\hline 2 & Second & Left & 8 & 100.283 \\
\hline 3 & First & Right & 8 & 100.05 \\
\hline 3 & Second & Left & 8 & 125.13 \\
\hline 4 & First & Left & 8 & 134.6 \\
\hline 4 & Second & Right & 8 & 139.53 \\
\hline & First & Right & 8 & 129.5 \\
\hline 5 & Second & Left & 8 & 125.4 \\
\hline
\end{tabular}




\section{APPENDIX G}

\section{Muscular Branch Functional HyPeremia Data For Ligated Mice}

Table 2. Muscular branch functional hyperemia data for ligated mice.

\begin{tabular}{|c|c|c|c|c|}
\hline Mouse & Surgery Type & $\begin{array}{c}\text { Stimulation } \\
\text { Frequency }(\mathrm{Hz})\end{array}$ & $\begin{array}{c}\text { Muscular Branch } \\
\text { Arterial Diameter } \\
(\mu \mathrm{m})\end{array}$ & $\begin{array}{l}\text { Vasodilation } \\
\text { (\% Increase) }\end{array}$ \\
\hline 1 & Sham & 0 & 58.45 & \\
\hline & Sham & 0 & 69.52 & \\
\hline 3 & Sham & 0 & 59.08 & \\
\hline 4 & Sham & 0. & 62.14 & \\
\hline & Sham & 0 & 54.5 & \\
\hline 6 & Sham & 0 & 64.4 & \\
\hline 1 & Sham & 2 & 78.42 & 34.16595381 \\
\hline 2 & Sham & 2 & 93.77 & 34.88204833 \\
\hline & Sham & 2. & 73.4 & 24.23832092 \\
\hline 4 & Sham & 2 & 96.49 & 55.2784036 \\
\hline & Sham & 2 & 85.01 & 55.98165138 \\
\hline 6 & Sham & 2 & 104.95 & 62.96583851 \\
\hline 1. & Sham & 4 & 62.15 & 6.330196749 \\
\hline & Sham & 4 & 111.69 & 60.65880322 \\
\hline 3 & Sham & 4 & 66.28 & 12.18686527 \\
\hline 4 & Sham & 4 & 95.16 & 53.13807531 \\
\hline & Sham & 4 & 75.19 & 37.96330275 \\
\hline 6 & Sham & 4 & 104.31 & 61.97204969 \\
\hline 1 & Sham & 8 & 102.7 & 75.70573139 \\
\hline 2 & Sham & 8 & 103.91 & 49.46777906 \\
\hline & Sham & 8 & 102.68 & 73.79823968 \\
\hline 4 & Sham & 8 & 109.26 & 75.82877374 \\
\hline 5 & Sham & 8 & 109.44 & 100.8073394 \\
\hline & Sham & 8 & 107.6 & 67.08074534 \\
\hline & Ligation & 0 & 66.72 & \\
\hline & Ligation & 0 & 82.38 & \\
\hline 3 & Ligation & 0 & 79.72 & \\
\hline & Ligation. & 0 & 68.15 & \\
\hline 5 & Ligation & 0 & 59.5 & \\
\hline 6 & Ligation & 0 & 75.63 & \\
\hline 1 & Ligation & 2 & 90.26 & 35.28177458 \\
\hline & Ligation & 2 & 116.99 & 42.01262442 \\
\hline 3 & Ligation & 2 & 95.68 & 20.02007025 \\
\hline 4 & Ligation & 2 & 95.22 & 39.72120323 \\
\hline 5 & Ligation & 2 & 81.46 & 36.90756303 \\
\hline 6 & Ligation & 2 & 134.37 & 77.66759223 \\
\hline 1 & Ligation & 4 & 70.94 & 6.324940048 \\
\hline 2 & Ligation & 4 & 93.97 & 14.06894877 \\
\hline 3 & Ligation & 4 & 92.16 & 15.60461616 \\
\hline 4 & Ligation & 4 & 80.82 & 18.59134263 \\
\hline 5 & Ligation & 4 & 90.91 & 52.78991597 \\
\hline 6 & Ligation & 4 & 123.13 & 62.80576491 \\
\hline 1 & Ligation & 8 & 115.62 & 73.29136691 \\
\hline & Ligation & 8 & 120.64 & 46.44331148 \\
\hline & Ligation & 8 & 108.98 & 36.70346212 \\
\hline 4 & Ligation & 8 & 112.52 & 65.10638298 \\
\hline 5 & Ligation & -8 & 120.34 & 102.2521008 \\
\hline 6 & Ligation & 8 & 141.95 & 87.69007008 \\
\hline
\end{tabular}




\section{APPENDIX H}

\section{Muscular Branch Functional Hyperemia Data for Resected Mice}

Table 3. Muscular branch functional hyperemia data for resected mice.

\begin{tabular}{|c|c|c|c|c|}
\hline Mouse & Surgery Type & $\begin{array}{c}\text { Stimulation } \\
\text { Frequency }(\mathrm{Hz})\end{array}$ & $\begin{array}{c}\text { Muscular } \\
\text { Branch Arterial } \\
\text { Diameter }(\mu \mathrm{m})\end{array}$ & $\begin{array}{l}\text { Vasodilation } \\
\text { (\% Increase) }\end{array}$ \\
\hline & Sham & 0 & 64.82 & \\
\hline & Sham & 0 & 61.48 & \\
\hline & Sham & 0 & 71.11 & \\
\hline 4 & Sham & 0 & 55.66 & \\
\hline & Sham & 2 & 89.66 & 38.3215057 \\
\hline & Sham & 2 & 99.98 & 62.6219909 \\
\hline & Sham & 2 & 107.79 & 51.582056 \\
\hline 4 & Sham & 2 & 106.84 & 91.9511319 \\
\hline & Sham & 4 & 102.42 & 58.006788 \\
\hline$\therefore-$ & Sham & 4 & 112.78 & 83.4417697 \\
\hline & Sham & 4 & 123.76 & 74.0402194 \\
\hline 4 & Sham & 4 & 106.61 & 91.5379087 \\
\hline & Sham & 8 & 105.48 & 62.7275532 \\
\hline & Sham & 8 & 118.23 & 92.3064411 \\
\hline 3 & Sham & 8 & 124.18 & 74.6308536 \\
\hline 4 & Sham & 8 & 104.35 & 87.4775422 \\
\hline & Resection & 0 & 43.56 & \\
\hline & Resection & 0 & 64.28 & \\
\hline 3 & Resection & 0 & 43.48 & \\
\hline 4 & Resection & 0 & 61.35 & \\
\hline & Resection & 2 & 99.17 & 127.662994 \\
\hline & Resection & 2 & 113.29 & 76.2445551 \\
\hline 3 & Resection & 2 & 92.36 & 112.419503 \\
\hline & Resection & 2 & 95.29 & 55.3219234 \\
\hline & Resection & 4 & 83.27 & 91.1616162 \\
\hline & Resection & 4 & 109.37 & 70.1462352 \\
\hline & Resection & 4 & 93.82 & 115.777369 \\
\hline & Resection & 4 & 106.95 & 74.3276284 \\
\hline & Resection & 8 & 95.99 & 120.362718 \\
\hline & Resection & 8 & 112.01 & 74.253267 \\
\hline & Resection & 8 & 109.75 & 152.414903 \\
\hline 4 & Resection & $\begin{array}{r}8 \\
\end{array}$ & 103.4 & 68.5411573 \\
\hline
\end{tabular}

